Seton Hall University eRepository@Seton Hall

Seton Hall University Dissertations and Theses (ETDs)

Seton Hall University Dissertations and Theses

Summer 8-1-2015

\title{
Salivary VIP concentrations are elevated in humans after acute stress
}

Giovanni Ventre

giovanni.ventre@student.shu.edu

Follow this and additional works at: https://scholarship.shu.edu/dissertations

Part of the Other Physiology Commons

Recommended Citation

Ventre, Giovanni, "Salivary VIP concentrations are elevated in humans after acute stress" (2015). Seton Hall University Dissertations and Theses (ETDs). 2092.

https://scholarship.shu.edu/dissertations/2092 


\title{
Salivary VIP concentrations are elevated in humans after acute stress
}

\author{
By \\ Giovanni Ventre
}

Submitted in partial fulfillment of the requirements for the degree of Doctor of Philosophy in Molecular Bioscience from the Department of Biological Sciences of Seton Hall University 
(C) 2015 Giovanni Ventre 
APPROVED BY

Roberta L. Moldow, Ph.D. MENTOR

Kevin Beck, Ph.D.

COMMITTEE MEMBER

Jane L. Ko, Ph.D.

COMMITTEE MEMBER

Heping Zhou, Ph.D.

COMMITTEE MEMBER

Carolyn S. Bentivegna, Ph.D.

COMMITTEE MEMBER

Angela V. Klaus, Ph.D.

DIRECTOR OF GRADUATE STUDIES

Jane L. Ko, Ph.D.

CHAIRPERSON, DEPARTMENT OF BIOLOGY 


\section{Acknowledgements}

It is very difficult for me to express my sincere gratitude to my mentor, Dr. Roberta Moldow. It has been a privilege and honor to have been a part of her lab. I have learned a tremendous amount from her on how to be a good scientist. She has instilled in me a true appreciation of what stress research is all about. It goes without saying that I would not have been able to accomplish this important achievement in my life without her expertise and patience. Dr. Moldow's hard work and dedication were important factors for us being able to produce a publication. I will be forever grateful for her wisdom and leadership during my time as her graduate student.

I would also like to express my sincerest gratitude to my dissertation committee members: Dr. Kevin Beck, Dr. Jane Ko, Dr. Heping Zhou, Dr. Carolyn Bentivegna and Dr. Roberta Moldow. I would like to thank the entire committee for their time and patience in reviewing the project and for their helpful suggestions. Special thanks to Dr. Roberta Moldow and Dr. Kevin Beck for their many helpful insights and suggested improvements concerning the thesis manuscript. And finally, I would like to thank Dr. Jane Ko for her advice on formatting the thesis.

I would also like to thank the many students at Seton Hall University and all those who volunteered their time by supplying saliva samples for the different studies. I would also like to give special thanks to the fellow students that I had the opportunity to work with in Dr. Moldow's lab. I would like to recognize the following folks for their hard work for carrying out various aspects of the studies which may have included the salivary measurements of cortisol, DHEA, and amylase: Olivita Couso, Denise Alfano, Jennifer 
Smith, Caitlyn Colonna, Jessica Nicaretti, Michael Dean, Frank Zadlock and Richard Greendyk. And I cannot forget Luba Bululu for befriending me and teaching me the ropes with regards to the RIA and other important aspects of working in Dr. Moldow's lab.

The acknowledgements would not be complete if I did not thank Ms. Anjeanette Cook for all the help and advice she has given me over the years. She is one of the most approachable people I know, and I cannot imagine the Biology department without her. She is a living example of the University's core values.

Lastly, I am grateful for my whole family, who supported me with their unwavering kindness. And I would like to thank my niece, Lauren Fitzgerald, an educator in the field of biology for inspiring me to use my talents to pursue a career in teaching. 


\section{Table of Contents}

Title

Acknowledgement

List of Tables

List of Figures

Abstract

Introduction

Materials and Methods

Results

Discussion

References
Page

iii

vi

vii

viii

1

39

56

92

106 


\section{List of Tables}

Table 1. Demographics data for disaster drill stress (PAD).

Table 2. Demographics data for exam stress studies.

Table 3. Demographics data for training exercises (ROTC cadets).

Table 4. Demographics data for circadian rhythm, water intake and coffee intake studies.

Table 5. Percent of NPY recovery using different preservative mixtures.

Table 6. Percent of VIP recovery using different preservative mixtures.

Table 7. Preservation and determination of immunoreactive NPY and VIP using saliva samples spiked with known amounts of cold NPY or cold VIP.

Table 8. Recovery of radioactive NPY and VIP in saliva after solid phase extraction.

Table 9. E1 Exam Group: Results of Repeated Measures ANOVA.

Table 10. E2 Exam Group: Results of Repeated Measures ANOVA.

Table 11. E3 Exam Group: Results of Repeated Measures ANOVA.

Table 12. E4 Exam Group: Results of Repeated Measures ANOVA.

Table 13. E5 Exam Group: Results of Repeated Measures ANOVA.

Table 14. Exam Stress tension: Results of Repeated Measures ANOVA.

Table 15. Results for Training exercise 1: Linear regressions.

Table 16. Results for Training exercise 2: Linear regressions.

Table 17. Results for Training exercise 3: Linear regressions.

Table 18. Borg results for Training Exercises 1,2, \& 3. 


\section{List of Figures}

Fig. 1 Diagram of the Hypothalamus-Pituitary-Adrenal Axis.

Fig. 2 Graphs of SWAT team exercise.

Fig. 3 Correlation between the ratio of DHEA/Cortisol and the degree of calmness.

Fig. 4 Disaster Drill study: salivary levels of stress markers.

Fig. 5 E1 Exam Group: salivary levels of stress markers.

Fig. 6 E2 Exam Group: salivary levels of stress markers.

Fig. 7 E3 Exam Group: salivary levels of stress markers.

Fig. 8 E4 Exam Group: salivary levels of stress markers.

Fig. 9 E5 Exam Group: salivary levels of stress markers.

Fig. 10 Training exercise 1: salivary levels of stress markers.

Fig. 11 Training exercise 2: salivary levels of stress markers.

Fig. 12 Training exercise 3: salivary levels of stress markers.

Fig. 13 Circadian studies: salivary levels of stress markers.

Fig. 14 Water intake studies: salivary levels of stress markers.

Fig. 15 Coffee intake studies: salivary levels of stress markers. 


\section{Abstract}

The purpose of this research was to evaluate two salivary neuropeptides in the context of the stress response. The research was focused first and foremost on evaluating the usefulness of salivary Neuropeptide Y (NPY) and salivary vasoactive intestinal peptide (VIP) as stress indicators. Secondly, we questioned whether these markers help us in distinguishing between physical and psychological stress. Psychological stress can be experienced in a situation such as that in anticipation of an important exam or mental task. Physical stress can be experienced usually by strenuous exercise. Salivary NPY and salivary VIP are two neuropeptides that are released as a result of sympathetic and parasympathetic activity respectively. These two biomarkers are measured extensively in the blood or plasma but they are not routinely measured in saliva (Morgan et al., 2000). These neuropeptides will be evaluated in conjunction with the following salivary indicators cortisol, dehydroepiandrosterone (DHEA) (Morgan et al., 2004) and amylase (Nater et al., 2006) of the stress response. Depending on the stressful situation or stress paradigm, we used an indicator or biomarker profile in conjunction with the use of psychological assessments in order to measure the intensity of the stress response. In other situations, the biomarker profile with the aid of psychological and physical assessment tools helped us differentiate between psychological stress and physical stress. In essence, we used a matrix approach using a combination of physiological and psychological measures to develop a stress profile that could be used to determine both the degree and the nature of the stress response experienced by subjects. In this study we evaluated NPY and VIP as salivary biomarkers using different stress paradigms. We also 
studied the effects of the following possible confounders on the stress response: circadian variation, water, and coffee intake. Therefore, to accomplish this salivary samples were taken and s-cortisol, s- DHEA, s-amylase, s-NPY and s-VIP were measured. Assessments (Thayer Activation-Deactivation Checklist, State-Trait Anxiety Inventory, and Borg Rating of Perceived Exertion Scale) were also administered in order to evaluate the psychological state of the subjects with respect to anxiety and their perception of physical exertion. The main findings of the study indicate that a brief intense exercise lasting minutes elicited a stress response with significant increases in s-cortisol, s-DHEA, s-amylase and s-VIP. A less rigorous exercise did not elicit a stress response with no significant increases in s-cortisol, s-DHEA,s-amylase and s-VIP. A longer intense exercise lasting hours elicited a stress response with significant increases only in scortisol. In addition, s-VIP displays a circadian variation which peaks with cortisol at 0800h. 


\section{Introduction}

Physiological stress is the body's response to a stressor such as an external or internal environmental factor which is experienced by everyone under certain situations. The stress response is mediated by the autonomic nervous system (ANS) and the hypothalamic pituitary adrenal (HPA) axis. The peripheral effects on physiology mediated by the HPA axis and the ANS is what we know as the stress response. The word stress was made popular by Hans Selye who was a pioneer in the stress field especially in relation to the HPA axis. Stress is an intriguing topic not only because it challenges homeostasis but because it has health implications especially when the stress system undergoes chronic activation. Researchers study stress in order to evaluate its detrimental effects on homeostasis. Indeed syndromes such as depression, chronic fatigue syndrome and post-traumatic stress disorder are believed to develop from chronic or even acute stress. (Chrousos and Gold, 1992) In addition, studying the stress response when personnel are undergoing certain training exercises can be useful to determine if the training exercises are effective. Because increased stress hormones can influence the way an individual responds to a situation, effective training will increase stress hormones so that the trainees will be prepared for their effects. Such stress studies are not only useful for the military but also for training law enforcement and emergency response personnel.

Nevertheless, in order to truly define stress one must define it physiologically and an active area of research for many years has focused on physiological measurements of stress such as cortisol. Cortisol is a marker for the HPA-axis and the catecholamines, 
norepinephrine and epinephrine are markers for the sympathetic branch of the ANS. According to Nater et al., salivary measures have become more important in psychoneuroendocrinological research and especially stress research. (Nater et al., 2009). However, work still needs to be done in this field and indicators which could help define or characterize the stress response in saliva are needed. Salivary Cortisol is an excellent marker for the HPA-axis activity, but the catecholamines cannot be detected in saliva. Salivary amylase, a starch digestive enzyme, has been used as a marker of the sympathetic division of the ANS. In addition to salivary-cortisol, salivary-DHEA (dehydroepiandrosterone) has also been measured in the context of stress paradigms (Morgan et al., 2004). Since stress is a multifaceted phenomenon, new markers can be useful for using a marker profile approach to describing the stress response. Several markers may provide more information about how much stress an individual is experiencing vs. using just one stress biomarker. Saliva also holds the possibility of assaying other markers such as NPY and VIP which are co released from sympathetic and parasympathetic nerves respectively.

The term "stress" can be broken down into 2 terms: stressor and stress response (McEwen, 2002). A stressor can be considered any factor which can alter homeostasis. When an individual is exposed to a physical or psychological stressor, the body reacts with the stress response which is also the known as the "fight or flight" response. The "fight or flight" response is carried out by the HPA axis and the sympathetic branch of the ANS. The parasympathetic branch of the ANS then responds to balance the 
sympathetic branch. In order to understand stress one must understand the changes that occur to homeostasis or how the body maintains normal physiology.

The stress response is part of a physiological coping mechanism that involves the HPA axis and the ANS, and the cardiovascular, metabolic and immune systems, and its job is to protect the body from internal or external stress (McEwen, 2002). "Physiological changes occur regardless of whether the stress is positive (rewarding) or negative (threatening)" (Herman et al., 1997). Therefore, challenges that lead to a feeling of exhilaration such as physical exercise could be regarded as "good stress". However, stress usually refers to an event or a series of events that challenge homeostasis and cause a response often in the form of distress (McEwen, 2002). The physiologic changes that occur can be regarded as adaptive by the organism. The stress response refers to the physiological changes that occur in the body in order to maintain homeostasis.

Stressors can be either physical or psychological in nature (McEwen, 2002). We are most familiar with physical stress such as strenuous exercise, however, psychological stressors are considered among the most powerful stressors. Examples of psychological stressors could include situations which contain a component of the following: novelty, withholding of reward and anticipation of punishment. These situations can be among the most potent activators of the HPA axis and the ANS (McEwen, 2002). Hormonal and tissue mediators that are released in response to stressful challenges produce cellular responses throughout the body that can be protective in the short run but can be damaging if the mediators are overproduced (McEwen, 2002). In fact, an active area of research today is to elucidate the factors that are either overproduced or under produced in many 
CNS disorders such as depression, post traumatic stress disorder (PTSD) and chronic fatigue syndrome (DeKloet et al., 2006; Jerjes et al., 2005).

The stress response is important for the organism and has an essential purpose: the survival of the organism. For example, the stress response has the following effects: mobilization of energy stores to maintain brain and muscle function, focuses attention on the perceived threat, increases blood flow to the brain, increases heart rate and cardiovascular output, inhibits immune reactions (inflammation), inhibits reproductive physiology and decreases feeding and appetite. All of these responses have the effect of diverting the body's resources in order to address the immediate threat. In essence these responses have the purpose of preserving homeostasis in the time of emergency.

The ANS is divided into two divisions (sympathetic and parasympathetic). The sympathetic division is active when homeostasis has been disrupted by stress and the parasympathetic division counteracts that of the sympathetic division in order to maintain or restore homeostasis (Recordati et al., 2003). The parasympathetic division is also active in times of rest. Activation of the sympathetic nervous system has the effect of helping the body meet the energy and physical demands of the stress response (Recordati et al., 2003). It is important to note that the sympathetic system originates from the thoracic and upper lumbar spinal segments and is therefore called the thoracolumbar system. The parasympathetic system originates from the brain stem (medulla oblongata, mesencephalon) and sacral spinal cord and is therefore called the craniosacral system.

One cannot mention stress without mentioning heart rate. Since heart rate is under autonomic control both physical and psychological stress can affect heart rate 
through the sinoatrial (SA) node. Under conditions of rest, the parasympathetic fibers release acetylcholine, which acts to slow the pacemaker potential of the SA node and thus reduce heart rate. Under conditions of physical or emotional activity sympathetic nerve fibers release norepinephrine and epinephrine from the adrenal medulla which act to speed up the pacemaker potential of the SA node thus increasing heart rate. In addition the SA node is directly enervated by the sympathetic neurons. This is experienced by anyone who has been on a treadmill for an hour or has waited anxiously for the results of an important college exam. The parasympathethic system increases during stress to provide balance to the increased sympathetic nervous system and bring the heart rate back down (Porges et al., 1995). Cardiac vagal tone has been described as a novel index of stress and stress vulnerability with emphasis on the role of the parasympathetic system and particularly the vagus nerve in defining stress (Porges et al., 1995).

Along with the sympathetic nervous system there is the activation of the HPAaxis (Figure 1). The hypothalamus receives input from areas of the brain monitoring physiological parameters and emotional responses. Corticotropin releasing hormone $(\mathrm{CRH})$ is released from the hypothalamus, travels through the portal circulation to the anterior lobe of the pituitary gland to release Adrenocorticotropin hormone (ACTH) (Carrasco et al., 2003). ACTH is the key regulator of glucocorticoid secretion from the adrenal cortex (Chrousos and Gold, 1992). Minutes after ACTH is secreted from the pituitary, there is an increase in plasma levels of glucocorticoids from the adrenal cortex which peak 30 minutes to 1 hour later (Carrasco et al. 2003). Glucocorticoids play a principal role in energy metabolism, growth processes and immune function. 
Glucocorticoids also participate in the learning and memory processes underlying behavioral adaptation as do catecholamines released from the medulla. Glucocorticoids also play a key regulatory role in the neuroendocrine control of the HPA axis and on the termination of the stress response by exerting negative feedback at the level of the hypothalamus and pituitary (Chrousos and Gold, 1992) (Figure 1).

Stress is usually defined by elevated cortisol. Elevated cortisol indicates activation of the HPA axis. However, cortisol may not be the only molecule which conveys information during the stress response. For example, strong correlations have been made between cortisol and DHEA during exposure to either psychological stress or physical stress. Both cortisol and DHEA are produced in the adrenal cortex. One can hypothesize that a person is exposed to varying degrees of external stimuli in which some may elicit a stress response and others will not. Consider the task of distinguishing biomarkers for physiological and psychological stress and one can see the difficulties in measuring the level of stress. In addition, we have now just begun to understand how the immune system can affect the HPA axis. NPY and VIP are two well-studied neuropeptides and agonists and antagonists are currently in development for a variety of disease states. However, there is a scarcity of information with regards to the involvement of these two neuropeptides in the stress response only from the standpoint of saliva. 


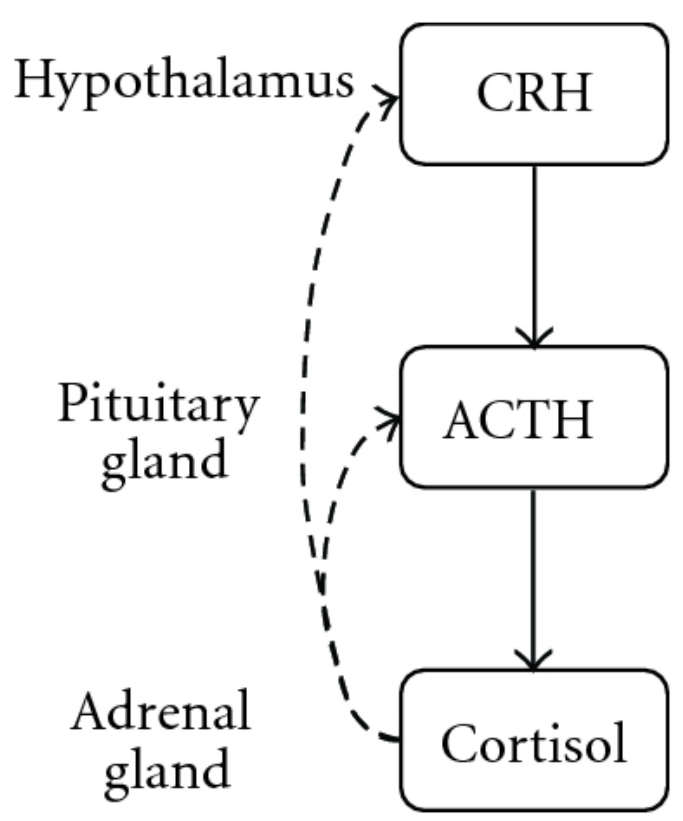

Fig. 1 Diagram of the Hypothalamus-Pituitary-Adrenal Axis 


\section{Stress Profile Approach}

Based on the anatomical considerations of the ANS and HPA axis it may not be possible to distinguish between the types of stress experienced: psychological vs. physical. "However the brain is the key organ of the adaptive and maladaptive responses to stress because it determines what is threatening and therefore potentially stressful, as well as initiating the behavioral as well as many of the physiological responses to the stressors, which can be either adaptive or damaging. Stress involves two way communications between the brain and the cardiovascular, immune and other systems via the autonomic nervous system and via endocrine systems"(McEwen, 2009). To elaborate on this point further, the stress response has three basic phases: the first phase is characterized by the rapid release of catecholamines via the autonomic nervous system; a few minutes later the second phase is characterized by the release of glucocorticoids into the circulation and the third phase involves the response of organs to the catecholamines and glucocorticoids. (Lovejoy, 2005). Therefore, trying to evaluate possible markers such as NPY and VIP from saliva may be a feasible measure of autonomic activation. Scientists such as Stuart McDougall also believe that that the CNS system can differentiate between physical and psychological stress. "Evidence for such CNS differentiation of stressor types and the sequence of CNS information processing is exemplified by studies that have mapped early gene expression after physical or psychological stress exposure and intervention type experiments, like that of Li et al., who demonstrated that severing the catecholamine input to the hypothalamus from the brainstem prevented HPA axis activation in response to a physical but not psychological 
stressor" (McDougall et al., 2005). However other studies do show that catecholamines respond to both physical and psychological stressors. In addition studies in rats may suggest that "emotional stress utilizes glucose for energy, whereas physical stress tends to utilize fatty acids as well as glucose" (Lovejoy, 2005).

According to Nater et al. (2009), stress is a multi-faceted phenomenon and it requires a multidimensional measurement approach. Nater et al. (2009) further adds that research can gain from additions to the accepted biomarkers for psychobiological parameters. In order to effectively study the stress response, one must evaluate markers of both the autonomic nervous system and the HPA axis. Morgan et al. (2000) have also used this profile approach to studying the stress response. Noninvasive markers are found in saliva with cortisol being the most well studied and most referenced. Indeed correlations between free cortisol in the plasma and salivary cortisol have been observed. Other salivary markers are gaining popularity and credibility with regard to the stress response e.g. DHEA and amylase. For example, salivary amylase has been studied as a possible marker for sympathetic nervous system activation and research is ongoing.

An active area of research in our laboratory is the development of a profile which can distinguish between physical and psychological stress. Dr. Moldow's lab has already performed studies analyzing biomarkers (cortisol, DHEA and amylase) and heart rate responses to stress. An important principle of the research concerning emergencies and combat is that training under stress will translate into better performance in actual situations under stress. While this is a commonly accepted theory, how exactly is stress defined and quantified? I have already mentioned stress has many facets. Stress can 
adversely impact health and well being, and past efforts to quantify stress have fallen short, in part, because of an assumption or desire that a single measure could capture the subtleties of stress. Our working hypothesis is that the magnitude of stress can be quantified by a stress profile consisting of multiple measures including s-NPY and s-VIP and it may also be possible to distinguish physical (exercise) from psychological stress (Kraemer et al., 2005). To illustrate the importance of using multiple measures we will consider the following data related to SWAT exercises. One of the exercises used personnel in a building entry exercise whereby they would encounter possible threats and react to them appropriately. The encounter or even the anticipation of possible threats could elicit a stress response. In the other exercise, personnel had to drag an object mimicking a 150 pound body for 50 yards. As seen in Figure 2, cortisol increases following either the psychological stress of building entry or the physical stress of body drag (150 pounds for 50 yards) (Moldow et al., 2006). This clearly demonstrates that cortisol alone cannot be used to distinguish between psychological and physical stressors. Our lab has already discovered some interesting findings with respect to DHEA and cortisol. A relationship has been observed several times in which DHEA positively correlates with cortisol in response to different stress paradigms (physical and psychological stress). Another interesting finding from our lab revealed a correlation between the ratio of DHEA/Cortisol and the degree of calmness reported by the research subjects (Figure 3) (Moldow et al., 2006). The neuropeptides NPY and VIP have many functions attributed to them. Review of the literature suggests that higher levels of both peptides in saliva are associated with stress (Bellamy et al., 2006; Dawidson et al., 1997; 
Naito et al., 2003; Santavirta et al., 1997). The possibility that the addition of these two peptides to our profile will help distinguish between psychological or physical stressors remains to be elucidated.

In addition, salivary markers for autonomic activation may be useful measures for the stress response. Traditionally plasma norepinephrine has been measured as an indicator of sympathetic tone (Carrasco et al., 2003). Norepinephrine (NE) has not been detected in the saliva. However, salivary amylase has been considered to be an indicator of sympathetic activation. NPY is co released with norepinephrine and salivary NPY has been detected according to the published literature (Dawidson et al., 1997; Naito et al., 2003; Santavirta et al., 1997). It should be noted that the salivary levels of NPY reported in the literature are very low. Acetylcholine is the traditional indicator of parasympathetic activation however it is not detectable in saliva. VIP is co-released with acetylcholine from parasympathetic nerves which makes it a good indicator of parasympathetic activity or tone (Dawidson et al., 1997; Naito et al., 2003; Santavirta et al., 1997). In addition a statistically significant correlation between salivary NPY and VIP may indicate how well a subject responds to stress since these two hormones have opposite effects with respect to vagal tone. 

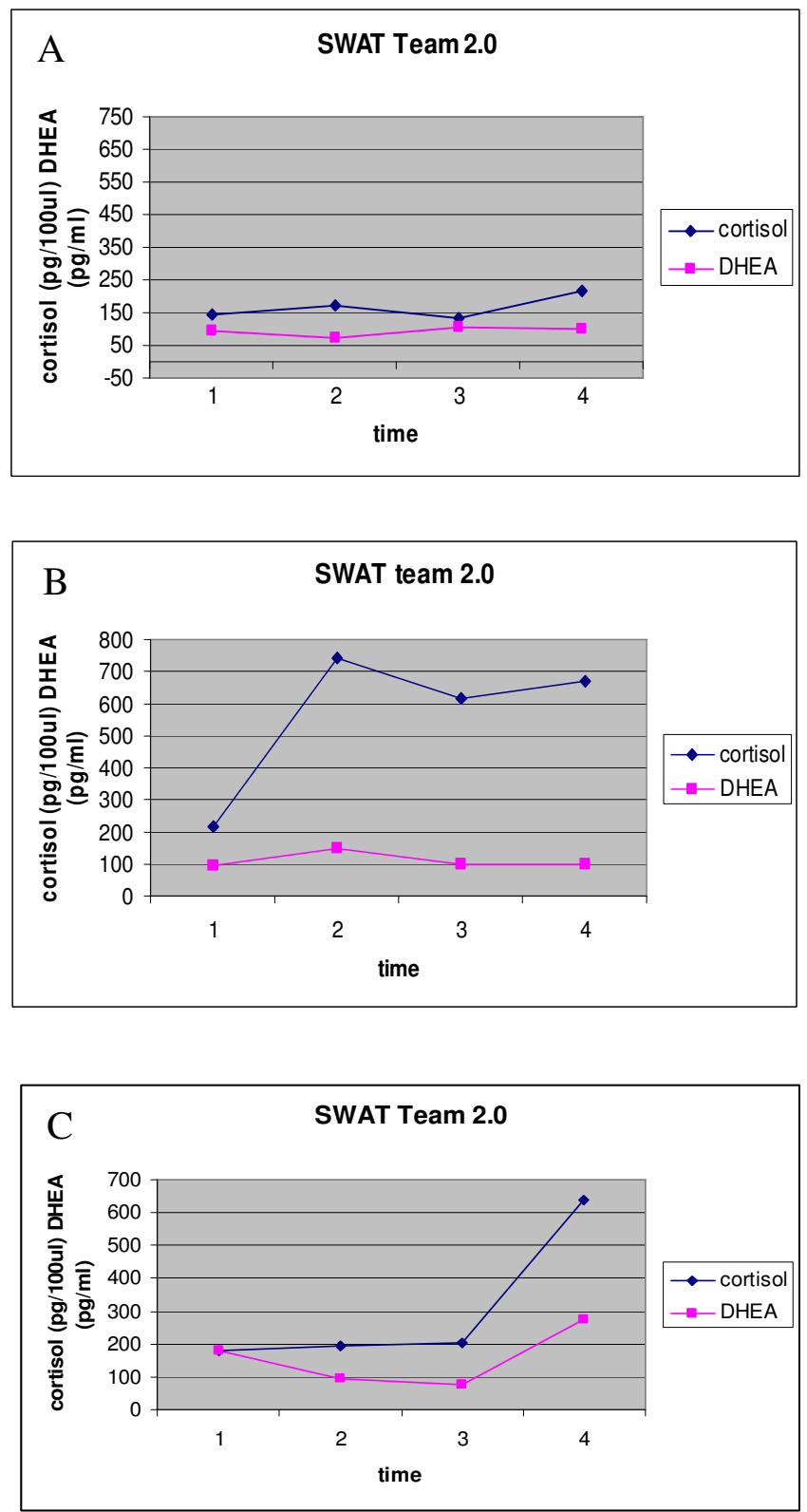

Fig. 2 (A) SWAT team exercise, subject 1 no response to either building entry (time 2) or body drag (times $3 \& 4$ ). (B) subject 2 response to both building entry (time 2) and body drag (time $3 \& 4$ ). (C) subject 3 response to body drag (time 4). The figure was adopted from Moldow et al., (2006). 


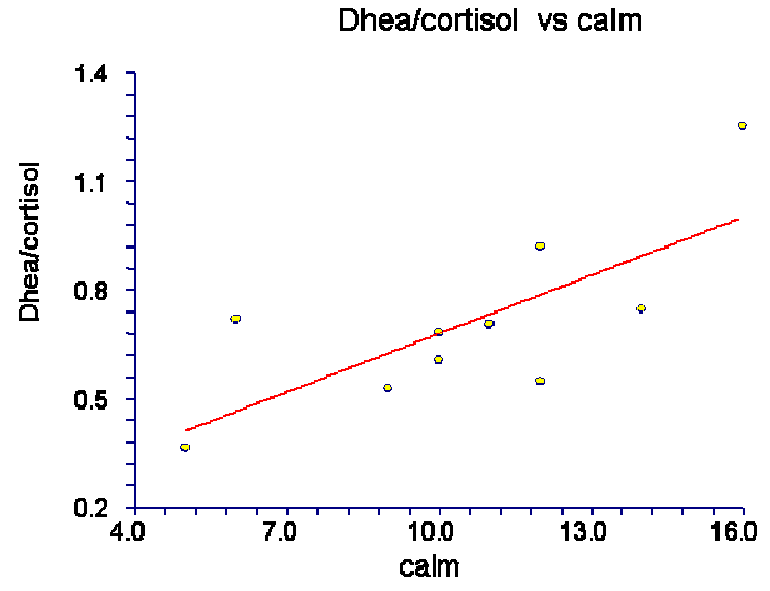

Fig. 3 Correlation between the ratio of DHEA/Cortisol and the degree of calmness reported by the research subjects. Linear regression results: $\mathrm{R}^{2}=0.5437, \mathrm{~F}=9.53$, $\mathrm{p}=0.0150$. The figure was adopted from Moldow et al. (2006). 


\section{Saliva}

Saliva is the main product of the salivary glands. Saliva collection and analysis is currently being used in a variety of fields including the stress field and sports training (Papacosta et al., 2011). Saliva provides a useful and non-invasive alternative to the collection of serum and plasma. It can be collected rapidly, frequently and without stress (Papacosta et al., 2011; Takai et al., 2004). There are 3 major salivary glands on each side of the face: the parotid, submandibular, and sublingual glands. Also, numerous minor glands contribute to salivary flow. The salivary glands are part of the digestive tract. The salivary glands are duct containing exocrine glands which produce and secrete a large volume of fluid containing organic and inorganic substances (Papacosta et al., 2011). The salivary glands secrete water, ion and proteins. Among the proteins found in saliva are proteins which protect the oral mucosa such as mucins and salivary-IgA, histatins, statherins and amylase. There are also hormones which are detected in saliva: cortisol, DHEA, melatonin, NPY, VIP, substance P, CGRP, and NKA (Dawidson et al., 1997).

Autonomic nerves control the secretion of salivary fluid and proteins (Proctor et al., 2007). Salivary glands are enervated by both parasympathetic and sympathetic nerves. Increases in parasympathetic nerve activity stimulate salivary glands to increase salivary flow (Proctor et al., 2007). Increases in sympathetic nerve activity also cause an increased release of proteins from salivary glands (Proctor et al., 2007). Among the neurotransmitters which are found in saliva, adrenaline and acetylcholine are released 
from the sympathetic nerves and parasympathetic nerves respectively (Proctor et al., 2007) However, van Stegeren et al. (2006) states that direct measurements of adrenaline and noradrenaline out of saliva seem not to reflect SAM activity. There are also nonadrenergic non-cholinergic (NANC) transmitters within nerves in salivary glands. Among the NANCs are NPY, NKA, substance P, VIP, PACAP, nNOS, and CGRP (Proctor et al., 2007; Dawidson et al., 1997; Marini et al., 2000). And specifically, both NPY and VIP have been detected in the autonomic nerve fibers of human labial salivary glands (Konttinen et al., 1992; Feher et al., 1999).

\section{Salivary Cortisol as a marker for stress}

Salivary cortisol is frequently used as a biomarker of stress. Most studies consider salivary cortisol levels as a reliable measure of hypothalamus pituitary adrenal axis adaptation to stress. Most studies report a high correlation between free cortisol levels in blood and saliva. A review of the literature suggests cortisol responds to both physical and psychological stress (Hellhammer et al., 2009). The literature also suggests that the level of salivary cortisol peaks around 20 minutes after the initiation of stress (Hellhammer et al., 2009; Gaab et al., 2003; Kudeilka et al., 2009)

Salivary cortisol is known to rise in response to physical stress (Papacosta et al., 2011). Blood lactate levels have also been known to rise significantly during physical exertion. Significant positive correlations have been made between blood lactate and cortisol (Papacosta et al., 2011). Indeed, salivary cortisol monitoring in sports can indicate the stress response to physical exertion as in exercise or training (Papacosta et 
al., 2011). Hellhammer et al., 2009 states that there are advantages in the stress free collection of salivary cortisol (Hellhammer et al., 2009). Hellhammer et al.(2009) believes that salivary measures are the method of choice in stress research.

Psychosocial stress leads to a release of cortisol. The HPA axis has been proposed as a major pathway linking psychological stress to its negative consequences on physiology (Hammerfald et al., 2006). Increased levels of cortisol has been shown to have negative health consequences (Hammerfald et al., 2006). Hammerfald et al. performed an intriguing study in which subjects were exposed to the Trier Social Stress Test (TSST) which consists of a mental arithmetic task and free speech in front of an audience. The results clearly show a significant rise in salivary cortisol in both men and women. All of the subjects then underwent cognitive-behavioral stress management training. The subjects then took the TSST again and the results showed an attenuation of the cortisol responses to stress (Hammerfald et al., 2006). The cortisol response was attenuated probably because it was their second experience.

\section{Salivary DHEA as a marker for stress}

DHEA is the most important androgen secreted by the adrenal cortex and is a precursor for sex steroids (Izawa et al., 2008). As is the case with cortisol, the main secretagogue for DHEA is ACTH (Izawa et al., 2008). Salivary DHEA levels have been found to respond to physical stress and some believe that it is a better marker than salivary testosterone for measuring the anabolic hormonal response to exercise in both males and females (Papacosta et al., 2011). DHEA affects several metabolic and 
immunological functions and is associated with lower incidence of ischemic heart disease and mortality from cardiovascular disease. (Izawa et al., 2008). Also DHEA is supposed to affect some brain functions such as cognition and mood (Izawa et al., 2008). For example, Izawa et al. found that "acute psychosocial stress significantly increased salivary DHEA levels by an average of $60 \%$ and the response was moderately correlated to the cortisol response" (Izawa et al., 2008; Shirotsuki et al., 2009). In the same study, Izawa et al. found lower DHEA levels and elevated cortisol/DHEA ratio during a TSST. Both lower DHEA levels and elevated cortisol/DHEA ratio were correlated with increased negative mood during and after TSST (Izawa et al., 2008). These results are somewhat consistent with other studies which report an improvement in mood with pharmacological administration of DHEA (Wolkowitz et al., 1999; Rabkin et al., 2006). Morgan et al. (2004) adds that a growing body of research has provided evidence that DHEA is involved in an organism's response to stress and that it may provide beneficial behavioral neurotrophic effects. In an interesting paper Morgan et al. investigated plasma DHEA-s and cortisol levels in soldiers who were undergoing survival training and found both plasma DHEA and cortisol to be significantly increased (Morgan et al., 2004). The findings of the paper suggest that a higher DHEA/cortisol ratio was associated with better military performance. The participants in the study were either naval aviators or marines and thus were well trained. Nevertheless, this paper provided evidence that plasma DHEA-s levels are increased by acute stress and in addition Morgan suggests that the DHEA/cortisol ratio may be an index for how an individual is protected against the negative effects of stress (Morgan et al., 2004). In summary, the findings of the paper 
suggest that a higher DHEA/cortisol ratio was associated with better military performance (Morgan et al., 2004).

\section{Salivary amylase as a marker for stress}

Salivary alpha amylase is one of the most important enzymes in saliva (Nater et al., 2009) Salivary alpha amylase accounts for $40 \%$ to $50 \%$ of the total salivary glandproduced protein, and most of the enzyme is synthesized in the parotid gland ( $80 \%$ of the total) (Nater et al., 2009). It is a calcium-containing metalloenzyme that hydrolyzes the alpha-1,4 linkages of starch to glucose and maltose. It is known to be mainly involved in the initiation of the digestion of starch in the oral cavity, but it has also been shown to have an important bacterial interactive function and thus important for innate immunity (Nater et al., 2009).

Studies have been performed to determine if salivary amylase can be a useful indicator for stress in particular for the activity of the sympathetic nervous system. A review of the literature suggests that salivary amylase will peak 10 minutes after the initiation of stress (Nater et al., 2005; Nater et al.; 2006, Nater et al., 2009). The sympathetic branch of nervous system is activated within seconds with the release of catecholamines followed by an increase in heart rate and then metabolic changes which occur to meet the demands of the stress response (Lovejoy, 2005). The release of salivary amylase is considered one of the metabolic changes which occur during the stress response (Nater et al., 2009). Norepinephrine from sympathetic neurons is one of 
the main neurotransmitters that bind to both the alpha and beta-adrenergic receptors found on salivary glands to cause secretion of salivary amylase (Nater et al., 2009).

Chatterton et al. performed studies where the subjects were exposed to physical (running, exercise, exposure to heat and cold) and psychological (examination) stressors (Chatterton et al., 1996). The group investigated the concentration of amylase as a measure of adrenergic activity under several conditions of stress in human subjects. Increases were observed in both salivary alpha amylase and the plasma catecholamines, norepinephrine and epinephrine, in response to physical and in some cases psychological stressors (Chatterton et al., 1996). Regressions of salivary amylase on plasma norepinephrine concentration were significant for both exercise and examination protocols. The author adds that the fit of the regression was better after subtracting the control (unstressed) levels. Aerobic exercise induced a 3 fold increase in amylase and both norepinephrine and epinephrine in plasma were increased 5 fold over control levels (Chatterton et al., 1996). During the written examination, amylase and norepinephrine but not epinephrine concentration increased in parallel. However, the regression between amylase and norepinephrine was only significant after subtraction of control (unstressed) levels (Chatterton et al., 1996). The Chatterton group concluded that "salivary amylase concentrations are predictive of plasma catecholamine levels particularly norepinephrine under a variety of stressful conditions" (Chatterton et al.,1996). Nater et al. in contrast to Chatterton et al. did not find a strong association between amylase and catecholamines using the Trier Social Stress Test but rather found a positive relationship between amylase and sympathetic tone during psychological stress as measured by heart rate 
variability (Nater et al., 2006). Heart rate variability is the physiological phenomenon of variation in the time interval between heartbeats. Nater et al. offered an explanation for the discrepancy that was based on the nature of the stressor. The Chatterton et al. (1996) study used a combination of physical and psychological stressors. Nater et al. (2006) make an important point that Chatterton et al. observed a positive significant correlation between s-amylase and plasma norepinephrine only with the physical stressor. However, there was only a weak and non-significant correlation between s-amylase and norepinephrine using the examination protocol (Nater et al., 2006). As a result of these studies Nater et al. suggest that during physical stress, the mechanism responsible for increased catecholamines and s-amylase may be different than when the stressor is psychological in nature (Nater et al., 2006).

Another study by van Stegeren et al. also investigated the possibility that amylase correlates well with plasma catecholamines by using the non-selective beta blocker propranolol to explore its effect on salivary amylase levels (van Stegeren et al., 2006). The study therefore investigated the effects of a pharmacological manipulation of the sympathetic-adrenal-medullary system (SAM) when participants underwent a stressful procedure such as watching a highly negative emotional picture in a scanner. The main finding of the study determined that treatment with the beta-blocker propranolol blocked the stress induced increase of salivary amylase observed in the placebo group. "Heart rate and systolic blood pressure were also reduced by propranolol treatment therefore the experiment supports the idea that amylase levels reflect beta adrenergic activity in the humans" (van Stegeren et al., 2006). This experiment lends support to the idea that the 
activation of salivary amylase can be considered a serial process where the SAM system is activated first, followed by heart rate increase and then salivary amylase secretion.

Takai et al. (2004) examined the effects of psychological stressor and soother on the salivary cortisol and amylase levels in young adults and found that salivary amylase level was increased significantly and reacted more rapidly than cortisol by the psychological stressor suggesting that it is a better index of psychological stress. In addition, the soothing video significantly lowered the amylase level but it did not affect the cortisol level (Takai et al., 2004). This finding may also suggest a connection between heart rate and s-amylase activity. Both the van Stegeren and Takai studies were focused on psychological stressors and their findings may be somewhat contrary to that of Nater's conclusions.

\section{Salivary Neuropeptide $Y$ as a marker for stress}

Neuropeptide Y (NPY) is a 36 amino acid peptide that has been highly conserved throughout mammalian evolution (Strand 1999). NPY was originally isolated from the brain (Tatemoto et al., 1982). It belongs to the pancreatic polypeptide family and is colocalized and released with neurons containing norepinephrine (Carrasco et al., 2003; Lundberg et al., 1990). There is much evidence to support the fact that NPY is a sympathetic neurotransmitter and a stress mediator (Kuo et al. 2007; Zukowska-Grojec et al.; 1998, Helig et al., 2004). NPY is also known to be potent vasoconstrictor and neuromodulator (Zukowska-Grojec et al., 1998). In addition to being a vasoconstrictor, evidence suggests that it is also angiogenic (Zukowska-Grojec et al., 1998). 
Angiogenesis is the physiological process whereby new blood vessels form from preexisting vessels. Therefore it may play a role during tissue development and repair (Zukowska-Grojec et al., 1998). And as a side note NPY is also known to be an appetite stimulant. It is important to note that the many or pleiotrophic effects of NPY are mediated by multiple receptors, Y1-Y5 (Kuo et al., 2007). In addition, even though NPY and NE are often co-released from the sympathetic nervous system, it is believed that NE and adrenergic mechanisms are responsible for maintaining arterial blood pressure, transient increases in vasoconstriction and cardiac function (Kuo et al., 2007). NPY in contrast may play a role in the response to prolonged or intense stress such as exhaustive exercise (Kuo et al., 2007; Helig et al., 2004; Zukowska-Grojec et al., 1998).

Other studies in humans also suggest a role for NPY during stress. In one research study done on military personnel, plasma NPY levels were significantly increased compared to pre-study levels following interrogations and were significantly higher in special-forces soldiers compared to non-special forces soldiers (Morgan et al., 2000). In addition, 24 hours after the conclusion of survival training, NPY had returned to baseline in special-forces soldiers (Morgan et al. 2000). However, in non-special forces soldiers, plasma NPY was significantly lower than initial values. Also, in the study NPY was positively correlated with both cortisol and behavioral performance under stress (Morgan et al., 2000). In the same study, plasma NPY levels were measured in non-combat exposed veterans and exposed combat veterans with PTSD and in veterans without PTSD. The authors found that there is an association between elevated NPY and the resistance to, or recovery from the adverse effects of stress. The study showed 
elevated NPY concentrations in trauma exposed veterans without PTSD compared to veterans with PTSD (Morgan et al., 2000). In another study published by the same lab, the data replicated the previous study and demonstrated that acute stress elicited NPY release. This NPY release was positively associated with cortisol and norepinephrine release (Morgan et al., 2002). The study also showed that greater levels of NPY are associated with less psychological distress (Morgan et al., 2002). Morgan et al. (2002) suggests that NPY confers anxiolytic activity which means it is associated with less anxiousness. Cortisol, however, was found to be positively correlated with distress (Morgan et al. 2002). Heilig et al., (2004) also reports that NPY is active in a wide range of anxiety models and that NPY may have anxiolytic like actions (Heilig et al., 2004). In another study examining the levels of plasma NPY in veterans, Yehuda et al. found NPY levels to possibly represent a "biologic correlate of resilience to or recovery from the adverse effects of stress" (Yehuda et al., 2006). It is important to note that the Yehuda et al. conclusions were partly based on the Morgan et al. studies.

The available evidence does suggest a role for NPY during certain situations of stress. One of the goals of this study is to determine if NPY can differentiate between physical and psychological stress. In order to accomplish this we will try to assay NPY in saliva which is much less invasive than taking blood (Dawidson et al., 1997; Naito et al. 2003; Santavirta et al., 1997).

Salivary Vasoactive Intestinal Peptide as a marker for stress 
VIP is a 28 amino acid peptide originally discovered in lung and small intestine by Said and Mutt (Said and Mutt 1970). VIP was subsequently found to be co-released with acetylcholine from parasympathetic nerves and was later identified in the nervous system and endocrine systems acting as a neurotransmitter/neuromodulator (Strand, 1999, Culp and Richardson, 1996). VIP is a member of a group of regulatory peptides that include secretin and glucagon among others. VIP/Ach containing nerves are found at high concentrations in the adrenal glands, salivary glands and pancreas (Igarashi et al, 2011). VIP is involved in vasodilation and, therefore, it has a function in counteracting the vasoconstrictive effects of the sympathetic response (Igarashi et al., 2011; Lundberg et al., 1980). There is also evidence which suggests that VIP may play a role in the regulation of the HPA-axis (Nussdorfer et al., 1998). VIP may also play a role centrally where it is a major peptide involved in the suprachiasmatic nucleus of the hypothalamus, which is the circadian pacemaker (Vosko et al., 2007). However, it is important to note that VIP's role in the CNS may have no correlation with its role in the parasympathetic nerves.

Evidence that VIP is involved in the stress response was suggested because of the observation that VIP increases the release of ACTH from tumor cell lines of both mouse and human pituitary cells (Carrasco et al., 2003; Oliva et al., 1982). Other evidence from rats subjected to different stressors measured VIP changes in certain areas of the brain such as amygdala and hippocampus (Carrasco et al., 2003; Nakamura et al., 1992,1994) In addition, other evidence suggests that VIP is a potent stimulator of aldosterone and corticosterone secretion (Carrasco et al., 2003; Nussdorfer et al., 1987). 
Other research concerning VIP and stress in humans measured plasma VIP in response to prolonged fasting and involuntary sleep withdrawal and exercise (Oktedalen et al., 1983-1; Oktedalen et al., 1983-2; Crozier et al., 1988). In the first of two different publications, the Oktedalen et al. group studied plasma VIP levels in 24 Norwegian military cadets before and after a 6 day study which involved prolonged physical exercise, calorie deficiency and sleep deprivation. The cadets were separated into 3 groups:

Group 1: prolonged physical exercise, calorie deficiency and sleep deprivation Group 2: prolonged physical exercise, iso-caloric, and sleep deprivation Group 3: prolonged physical exercise, calorie deficiency and 3 hours extra sleep All 3 groups saw a 2 to 4 fold increase in plasma VIP levels with the most elevated levels throughout the 6 days being in group 1 (Oktedalen et al., 1983-1). However in this study, plasma VIP levels returned back to normal within 15 to 30 minutes after a meal or glucose ingestion (100g glucose solution) (Oktedalen et al., 1983-1). The second study by Oktedalen et al. involved 12 subjects divided into two groups which took part in a 90km cross-country ski race lasting approximately 6 hours. (Oktedalen et al., 1983-2). Group 1 received a glucose infusion immediately after the race and group 2 did not. The plasma VIP levels went up for both groups: Group 1 increased from a control value of 6 $\mathrm{pmol} / \mathrm{L}(20 \mathrm{pg} / \mathrm{mL})$ to a post race value $16.2 \mathrm{pmol} / \mathrm{L}(50 \mathrm{pg} / \mathrm{mL})$ and group 2 went from a control value of $4 \mathrm{pmol} / \mathrm{L}(13.3 \mathrm{pg} / \mathrm{mL})$ to a post race value of $33.7 \mathrm{pmol} / \mathrm{L}(112 \mathrm{pg} / \mathrm{mL})$. However, the interesting difference in the groups was seen after the race when group 1 was given a glucose drink which led to a normalization of plasma levels just 15 minutes 
after the infusion whereas group 2 levels remained elevated even 2 hours after the race was over (Oktedalen et al., 1983-2). The results of these 2 studies by the Oktedalen group caused the authors to hypothesize that increased VIP in response to prolonged strenuous exercise is related to the metabolic function of VIP. In other words because of the suppression of plasma VIP by glucose the increase in VIP seems to be an indicator of energy substrate need rather than a response to the physical exertion or physical stress (Oktedalen et al., 1983-2). Therefore, plasma VIP may have an energy mobilizing function. In these studies VIP was increased in the plasma in response to the stress used and the authors suggested a possible role for VIP in the hormonal metabolic response to stress. Additional work which supports Oktedalen et al., (1983) is a paper by Opstad who examined the VIP response to prolonged strain, sleep and energy deficiency (Opstad, 1987). Opstad (1987) reports the results of an experiment where the rise in plasma VIP is much greater in response to 30 minutes of cycling when subjects were first subjected to prolonged strain, sleep and energy deficiency. The rise in plasma VIP during the exercise was abolished if the subjects were given an infusion of glucose. This experiment does support the previous findings from Oktedalen et al. that VIP may have an energy mobilizing function during exercise.

It is important to consider how plasma VIP levels correlate with saliva levels. Most studies that measure plasma VIP use pmol/Liter to quantify VIP levels. Two independent research studies which measured plasma VIP in normal subjects, but not in the context of the stress response gave values of $40+/-22 \mathrm{pg} / \mathrm{mL}$ and $42.4+/-17.1$ 
pg/mL, respectively (Petkov et al., 2003; O’Dorisio et al., 2002). These pg/mL levels of VIP are similar to the levels observed in saliva.

VIP is also detectable in saliva (Dawidson et al., 1997; Naito et al., 2003;

Santavirta et al., 1997). In one study comparing patients with Sjogren's syndrome and normal healthy subjects, stress as assessed by a Jenkins Activity Survey was associated with higher salivary VIP concentrations (Santavirta et al., 1997). Another noteworthy study found elevated salivary VIP and salivary CGRP in rhinosinusitis and migraine patients (Bellamy et al., 2006). Both of these reports studied VIP as an indicator of parasympathetic nerve activation. Therefore, we undertook studies to evaluate if salivary VIP levels increase with different types of stress situations. If it does, the question is can it possibly be used as a biomarker indicating how a particular individual responds to stress? It will also be interesting to observe if VIP correlates with other markers of stress such as cortisol, DHEA or amylase.

\section{Academic exam stress}

Research has demonstrated that written examination and or public speaking can be good paradigms for psychological stress. This type of social-evaluative threat can activate the HPA axis (Preuss et al., 2010). Academic stress can be considered a good model of a natural stress situation in humans. A study by Al-Ayadhi et al. (2005) suggests that exam stress is a good paradigm to study changes in neurohormones. The study monitored several hormones in medical students before and after an exam and the results were compared to hormone levels on a non-exam day. Noteworthy changes were 
in plasma cortisol, ACTH and NPY which were all elevated significantly after exam stress (Al-Ayadhi et al., 2005). Another study reports elevated cortisol levels the day prior to and on the day of a university written examination (Preuss et al., 2010). In reference to the cortisol levels on the day of the exam, the salivary cortisol levels were significantly elevated before the exam but not after the exam (Preuss et al., 2010). The same group also studied the effect of oral presentation on salivary cortisol levels and the results revealed substantially elevated cortisol levels before and after the oral presentation (Preuss et al., 2010). Another study by Schoofs et al. (2008) studied the effect of public speaking examination in 40 undergraduate students. The group reports strong increases in both anticipatory salivary cortisol and amylase as well as a further increase post examination (Schoofs et al., 2008).

Other studies using the Trier Social Stress Test show significant increases for salivary amylase, cortisol, catecholamines and cardiovascular parameters such as heart rate between the stress and rest conditions (Nater et al., 2006). Another group investigated the effect of the TSST on salivary DHEA levels (Izawa et al., 2008). The group reports a significant $60 \%$ increase in salivary DHEA levels immediately after the TSST. It is these results along with others which suggest that the TSST is a good research model for inducing stress (Birkett et al., 2011).

This current research proposal is intended to fill part of the gap that exists in this field with respect to specific markers for stress. NPY and VIP are two good candidates for stress biomarkers because they are released due to sympathetic activation and parasympathetic activation, respectively. These two neuropeptides have been studied 
extensively with respect to stress, cardiovascular effects and appetite (NPY) and antiinflammatory effects (VIP) in plasma (Kuo et al. 2007; Nussdorfer et al. 1998). Their involvement in particular types of stress is not as well studied and there is an apparent lack of research publications with respect to how NPY and VIP levels change in the saliva in response to stress.

\section{Confounders of salivary stress measures}

Since we are measuring biomarkers of the HPA axis and of the ANS it is important to investigate possible modulators of these systems which confound the stress responses that are observed in the field or laboratory setting (Kudielka et al., 2009). The most common possible confounders of salivary markers that we investigated were: circadian variations, water or coffee intake. We designed studies to evaluate these factors on s-cortisol, s-DHEA, s-amylase and s-VIP.

\section{Circadian Rhythms and cortisol, DHEA, amylase and VIP}

Circadian variation is an important factor to consider when planning stress experiments (Kudielka et al., 2009; Rohleder et al., 2009). According to Strahler et al. (2010), almost every physiological system has some degree of circadian rhythm that can be influenced not only by light exposure and the typical wake-sleep cycle but also by factors like age, sex and stress. The suprachiasmatic nucleus (SCN) in the hypothalamus is the main circadian pacemaker, integrating endogenous and exogenous information. Circadian rhythms regulate the hormones of the HPA axis as well as the ANS (Strahler et 
al., 2010). Hormones of the HPA axis such as ACTH, cortisol and DHEA have a distinct circadian rhythm which is higher in the morning upon wakening and decrease the rest of the day. "Even the autonomic nervous system which is considered one of the main stress sensitive systems in humans shows a distinct circadian rhythm with sympathetic activity increasing significantly during the day and decreasing during the night, while the parasympathetic activity decreases during the day and increases during the night" (Strahler et al., 2010). Strahler et al. examined saliva upon awakening, $+30 \mathrm{~min}$. awakening, 11am, 3pm, and 8pm and observed that salivary alpha-amylase peaks around 3:00pm with the lowest values upon awakening (Strahler et al., 2010).

Cortisol is known to have a distinct circadian rhythm with levels being highest in the early morning and decreasing throughout the day (Ahn et al., 2007; Krieger et al., 1971). Hucklebridge et al. studied the circadian profile of both salivary cortisol and salivary DHEA in the same paper (Hucklebridge et al., 2005). In addition to studying the pattern throughout the day, the investigators also study the awakening response for both steroids. Cortisol has a pronounced awakening response and is commonly known as the cortisol awakening response or CAR (Hucklebridge et al., 2005). The data clearly shows this response for cortisol for the first 45 minutes after awakening. Hucklebridge et al. did observe the awakening response with cortisol but not with DHEA.

The circadian profile for DHEA has been studied less than cortisol. However, DHEA displays a similar pattern as cortisol. The primary secretogogue for both cortisol and DHEA is ACTH so one would expect that they follow a very similar pattern including in the post awakening burst. However, Hucklebridge et al. states that the 
difference is probably due to downstream regulation. Downstream cortisol is synthesized by 17 alpha hydroxylase activity and DHEA by 17,20-lyase activity of cytochrome p450c17, and therefore the dissociation between cortisol and DHEA in reference to the post awakening response is probably due to the differential regulation of these two enzyme activities (Hucklebridge et al., 2005). The authors raise an important point that because the dynamics between cortisol and DHEA are different in the early morning consideration must be given to collection time in relation to awakening in the assessment of cortisol/DHEA ratios (Hucklebridge et al., 2005).

The levels of salivary-amylase also vary throughout the day. Nater et al. (2007) performed a detailed study taking samples at wake up, $30 \mathrm{~min}$., and $60 \mathrm{~min}$. after wake up and then hourly measurements from 9:00am until 8:00pm. Nater et al. also measured salivary cortisol at the same time points. The results show the typical circadian pattern for cortisol, (high in the morning with a gradual decline throughout the day). In contrast, salivary amylase shows a decrease after awakening then a gradual increase with a plateau in the afternoon and early evening (Nater et al., 2007). Consistent with the above data, Strahler et al. also found alpha-amylase to peak in the early afternoon around 3:00pm (Strahler et al., 2010).

There are no studies examining the circadian rhythm of salivary VIP. Some studies have looked at the circadian variation of plasma VIP. Cugini et al. found that plasma VIP levels peaked at 8:00pm and were lowest at 12:00am in young adults (Cugini et al., 1992). The Cugini study also reports that the elderly had peak VIP levels at 6:00pm and were lowest at 12:00pm (Cugini et al., 1992). Another study by Rolandi et 
al. report significantly higher levels of plasma VIP at 12:00am and 4:00am (Rolandi et al., 1990). This data is in agreement with previous observations reported by Jorde and Burhol which found that plasma VIP levels were highest during the night (Jorde and Burhol, 1985). A more recent study by Lockinger et al. measured plasma levels of cortisol and neuropeptides every four hours and reports plasma VIP has a circadian variation with levels peaking at 2:50am (Lockinger et al. 2004). Therefore, several studies report higher plasma VIP levels during the night. Other studies highlight the importance of VIP in relation to the regulation of circadian variations. Evidence suggests that VIP is important for the normal functioning of the suprachiasmatic nucleus in the hypothalamus (Loh et al., 2008; Vosko et al., 2007; Piggins et al, 2003). A study performed in VIP knockout mice demonstrates that the circadian rhythms of ACTH and corticosterone are lost and the authors suggest a role for VIP in regulating the mammalian master clock (Loh et al., 2008). Vosko et al. suggests that VIP plays a key role in circadian timekeeping. The review suggests that VIP is used as signaling molecule by the neurons of the SCN in order to generate circadian oscillations (Vosko et al., 2007). However, the VIP in the SCN may not be linked to parasympathetic activity and thus have no relation to VIP in saliva.

Water Intake effects on cortisol, DHEA, amylase and VIP

A survey of the literature did not find any definitive effects of water consumption on salivary hormone levels. A publication by Davies et al. investigated the effect of beverages on the stimulation and viscoelasticity of saliva. "The viscoelasticity and 
secretion rate of stimulated saliva depends on food and beverage related stimuli" (Davies et al., 2009). The lab investigated several beverages and their effect on saliva parameters such as flow rate. The group investigated the following on saliva flow rate: Ice Tea, Fizzy Cola, Sparkling Water, Gum, Mint and Water. The results indicated that all the treatments stimulated saliva flow rate with Ice Tea and Fizzy Cola stimulating flow rate the most. The results also show that water stimulated saliva flow rate the least. It is important to mention that Davies et al. states that when collecting samples from the volunteers, it was important to wait at least 5 minutes between each sample being collected to let the mouth return to an unstimulated, resting state. With regards to collecting salivary substances, it is possible that something like saliva flow rate could possibly affect their levels. For example, researchers such as Schultheiss suggest that stimulating saliva in order to collect cortisol, progesterone, testosterone and other substances may interfere with their proper measurement. Schultheiss points out stimulating saliva with sugarless gum is undesirable as it altered the results of the study (Schultheiss, 2013). Schultheiss observed a decrease in salivary cortisol in the chewing gum group. Schultheiss further adds that chewing gum can add substances to saliva which interfere with the antibody binding of cortisol. A different study by Naumova et al. (2013) takes another stance on salivary flow rate. Naumova et al. concluded from their paper that acute stress has no impact on the salivary flow rate (Naumova et al., 2013). However, Naumova et al. also found in the same study that saliva had an increase in salivary protein content. In addition, a study by Rohleder et al. reported that "the psychosocial stress-induced increase in salivary alpha-amylase is independent of saliva 
flow rate" (Rohleder et al., 2006).

Coffee intake effects on cortisol, DHEA, amylase and VIP

In a U.S. polled population, $96 \%$ of adults have reported consuming caffeine at some time in their lives and $83 \%$ have reported to consume $186 \mathrm{mg}$ of caffeine per day (Lovallo et al., 2006). According to Lovallo et al. (2006), caffeine acts as an important modulator of cardiovascular and central nervous system activity. In addition, certain amounts of caffeine have been reported to elevate blood pressure for several hours after intake and this effect can occur both at rest and during exercise or mental stress (Lovallo et al., 2006). An interesting finding from Lovallo et al. (2006) showed that 250mg of caffeine did not actually elevate cortisol levels in the absence of stress. Lovallo's group observed an increase in salivary cortisol levels when caffeine was administered in conjunction with mental stress or exercise. In another experiment using medical students, a $3.3 \mathrm{mg} / \mathrm{kg}$ dose of caffeine caused a significant increase in salivary cortisol after an exam (Shepard et al., 2000). There was no increase in salivary cortisol when the students consumed caffeine and attended a normal lecture (Shepard et al., 2000). Salivary cortisol levels also were not increased in the group that received placebo and took the exam nor in the group that received placebo and attended lecture (Shepard et al., 2000). Therefore these studies suggest that caffeine does not elevate cortisol levels in the absence of stress. (Lovallo et al.,2006; Shepard et al., 2000)

Another study by Slivka et al. (2008) investigated the interaction of caffeine and carbohydrate supplementation during exercise while the investigators studied the effect of 
carbohydrate and caffeine during exercise on fat usage and glucose utilization. The study demonstrated that ingestion of caffeine $(800 \mathrm{mg}$ or $5.3 \mathrm{mg} / \mathrm{kg}$ ) during exercise increased salivary cortisol when it was not taken with carbohydrate. The increase in cortisol was abolished when carbohydrate was co-ingested with caffeine. The investigators suggested that the results were consistent with the role of cortisol as a stress hormone which responds to the demands placed on fuel homeostasis (Slivka et al. 2008). In contrast, a study using different types of beverages observed a significant decrease in salivary cortisol 30 and 60 min. after ingestion of 100mg of caffeine (Quinlan et al., 1997). They also observed a decrease in an anxiety index and increases in hedonic tone and energetic arousal (Quinlan et al., 1997). The effect of caffeine on cortisol is not clear based on the several studies. The discrepancy may be due to experimental design. For example, the times of saliva collection were different among the mentioned studies. In addition, the dose of caffeine used was different for each study. The difference in experimental design coupled with the idea that the cortisol response to stress and/or caffeine possibly varies between individuals may lead to inconsistent results.

DHEA is synthesized from the same biosynthetic pathway as cortisol and ACTH is a secretagogue for both the adrenocortical hormone; therefore, DHEA could hypothetically respond the same way as cortisol. However, cortisol and DHEA production are due to different enzymes therefore they both could be regulated differently downstream of ACTH action (Hucklebridege et al., 2005). Mackenzie et al. investigated the effect of 1 week of moderate caffeine intake on serum adrenocortical hormones cortisol and DHEA as well as on salivary melatonin in healthy young adults. The 
investigators found no effect of 1 week of 1x200mg doses of caffeine supplementation on serum cortisol and serum DHEA levels (Mackenzie et al., 2007).

Salivary alpha-amylase has been proposed as a marker for activity of the sympathetic nervous system (Rohleder \& Nater, 2009). Recent studies in support of this hypothesis have led to an increased number of researchers using amylase measurements in experiments (Rohleder \& Nater, 2009). "Caffeine ingestion is associated with enhanced sympathetic nervous system activity and increases plasma epinephrine and is widely used among athletes for its ergogenic properties” (Bishop et al., 2006). Bishop et al. studied the effect of caffeine on salivary alpha amylase activity and they performed an initial pilot study to determine any independent effect of caffeine ingestion on SIgA and amylase at rest. In the Bishop et al. (2006) study, subjects received $6 \mathrm{mg} / \mathrm{kg}$ caffeine per body weight and salivary measures were taken no sooner than $1 \mathrm{hr}$. after caffeine administration. The results showed an increase in alpha amylase activity 1 hour after caffeine ingestion, and there was no increase in saliva flow with caffeine ingestion. The effect of caffeine ingestion $1 \mathrm{hr}$. before cycling for $90 \mathrm{~min}$. produced even higher alpha amylase activity than at rest (Bishop et al., 2006).

\section{Peptidase and protease activity and their presence in saliva}

The salivary glands synthesize and release many different types of proteins. Among these different proteins and peptides are: amylase, histatins, lysozyme, IgA, angiotensin II, vasoactive intestinal peptide, neuropeptide $\mathrm{Y}$ and substance $\mathrm{P}$ (Marini et al., 2000). Among the large array of bioactive proteins in saliva are proteolytic enzymes. 
Albo et al. suggests that the presence of peptidases serve as a specific mechanism to regulate the concentration of peptides and in particular neuropeptides (Albo et al., 2002). Marini et al. demonstrates peptidase activity in whole saliva using leu-enkephalin as substrate (Marini et al., 2000, Marini et al., 2001). The work of Marini and Albo demonstrated that saliva has aminopeptidase, dipeptidylaminopeptidase and dipeptidycarboxypeptidase activity. (Marini et al., 2000, Albo et al., 2001). In addition Albo et al. reports that male saliva has higher aminopeptidase activity than female saliva (Albo et al., 2001; Albo et al., 2002).

It is also reported that saliva contains a class of peptidases called kalilikrein related peptidases which have the ability to hydrolyze histatins (Andrade et al., 2011). In addition, a paper by Konttinen et al. demonstrates the presence of neutral endopeptidase activity in human labial salivary glands (Konttinen et al., 1996). Another research article demonstrates the presence of exosome-like vesicles with dipeptidyl peptidase (DPP) IV activity in human saliva (Ogawa et al., 2008). Ogawa et al. (2008) demonstrated that the DPP IV was able to cleave substance $\mathrm{P}$ and glucose-dependent insulinotropic polypeptide (Ogawa et al., 2008).

The fact that whole saliva contains proteases and peptidases could potentially affect salivary diagnostic data. One paper by Thomadaki et al. (2011) demonstrated that it was important to place saliva on ice and that saliva proteins were stable for up to $1 \mathrm{hr}$. on ice (Thomadaki et al., 2011). The Thomadaki et al. (2011) paper also reported that a cocktail containing the following protease inhibitors: AEBSF, aprotinin, (pancreatic trypsin inhibitor), leupeptin, antipain, and EDTA could not prevent histatin 5, statherin, 
or PRP1 degradation (Thomadaki et al., 2011). The presence of peptidase activity in saliva is a concern and their possible effect on any results should be considered.

In these studies, we examined the effect of psychological stress (exams), a combination of physical and psychological stress (training exercise 1), mild physical stress (training exercise 2) and physical stress (training exercise 3) on salivary markers for stress. Training exercise 1 was a brief intense exercise lasting minutes which involved an obstacle course. Training exercise 2 was a moderate exercise which lasted 1 hour. Training exercise 3 was a 3-4 hour training exercise which involved a 12 mile hike with a backpack weighing 30-35 lbs. Salivary cortisol and DHEA are markers for the HPA component of the stress response and salivary amylase is a marker for the sympathetic branch of the HPA. Our working hypotheses for these studies are as follows: 1.) NPY and VIP will be released during psychological stress; 2.) NPY and VIP will not be released during routine physical exertion; 3.) NPY and VIP will be released during very strenuous physical exertion. We will also determine if salivary VIP and NPY correlate with the established salivary markers for stress. Lastly, we will determine if levels correlate with the level of stress the subjects are experiencing by using the appropriate psychological assessments. 


\section{Materials and Methods}

Consent

Samples and questionnaires were collected from volunteer subjects anonymously for all studies after the consent form had been read and any questions pertaining to the study were answered. The consent form remained unsigned, as this would be the only way to identify the subjects who were to remain anonymous. Subjects were also reminded that participation was voluntary and could stop at any time for any reason without penalty. All of this was performed in accordance with the proposal approved by the Institutional Review Boards (IRB) at Seton Hall University (SHU). In addition, a waiver of informed consent was obtained from the (IRB) at Seton Hall University.

\section{Procedures}

The subjects were asked to complete a questionnaire that asks about age, last meal consumed, caffeine consumed and smoking behavior because these factors could affect the chemicals in the saliva. Before and after the exam or exercise, a salivary sample was taken and the following assessments were given at the same times. Note that all data was assigned a code and not a name (or other personal identifiers). The State-Trait Anxiety Inventory (STAI form Y-2) was given in order to elicit information on anxiety as a state and trait (Speilberger 1983). The Borg Rating of Perceived Exertion Scale (RPE) asks about how hard they think the body is working (Borg 1998). Another questionnaire derived from Thayer's Activation-Deactivation Checklist (ADCL), asks about states such as tiredness, calmness, and exertion (Thayer, 1978). 
Subjects and setting:

Disaster drill stress: PAD

The disaster drill was a training exercise to prepare and train regional volunteer Community Emergency Response Teams (CERT) how to respond efficiently to an emergency. However, the subjects were not from the CERT teams, but the personnel organizing and running the drill. Salivary samples were collected before the initiation of the training exercise at approximately $0700 \mathrm{~h}$, and after completion of the drills, which was early afternoon $(1300 \mathrm{~h})$. The demographics for the Pad drill is in Table 1. 
Table 1. Demographics: disaster drill stress (PAD)

\begin{tabular}{|l|l|l|l|l|l|}
\hline Group & \# subjects & \# females & \# males & Age range & Mean \\
\hline PAD & 7 & 0 & 7 & $26-65$ & 46.3 \\
\hline
\end{tabular}




\section{Exam Stress}

The volunteers were Seton Hall University undergraduate students taking important exams for their major. Times of sample collection are as follows: E1 Exam Group: 0830 h (before exam) and 1200 h (after exam); E2 Exam Group: 1300 h (before exam) and $1430 \mathrm{~h}$ (after exam); E3 Exam Group: $1300 \mathrm{~h}$ (before exam) and $1430 \mathrm{~h}$ (after exam); E4 Exam Group: 1730 h (before exam) and 1900 h (after exam); E5 Exam Group: $1500 \mathrm{~h}$ (before exam) and $1700 \mathrm{~h}$ (after exam). Samples were obtained before and after the exam and at comparable times on a control day. Note the samples were coded in order to keep track of the samples. The demographics for exam stress are in Table 2. 
Table 2. Demographics: Exam Stress

\begin{tabular}{|l|l|l|l|l|l|}
\hline Group & \# subjects & \# females & \# males & Age range & Mean \\
\hline E1 Exam Group & 8 & 4 & 4 & $18-24$ & 20 \\
\hline E2 Exam Group & 10 & 8 & 2 & $19-21$ & 19.5 \\
\hline E3 Exam Group & 5 & 5 & 0 & $18-20$ & 18.9 \\
\hline E4 Exam Group & 4 & 2 & 2 & $20-22$ & 20.8 \\
\hline E5 Exam Group & 8 & 6 & 2 & $19-20$ & 19.4 \\
\hline
\end{tabular}




\section{Training exercise 1}

This study took place on a military training base where a group of trainees were running through an obstacle course. The course run was approximately 10-15 minutes long and involved strenuous activity, such as wall jumps, rope climbs, and inclined-wall scaling, and running between each obstacle. Two samples were collected, the first around 1200h and the second taken immediately at the end of the obstacle course, about 10 minutes after the start of the course (about 1630h). Demographics are in Table 3.

\section{Training exercise 2}

ROTC cadets participated in a moderate exercise routine which lasted approximately 1 hour. Exercises performed were moderate aerobic exercises: running, stretching, jumping in place etc. Saliva samples were collected before exercise $0700 \mathrm{~h}$ and after exercise 0800h. Demographics are in Table 3.

\section{Training exercise 3}

ROTC cadets participated in a 3-4 hour training exercise which involved a 12 mile hike with a back pack weighing 30 pounds. Saliva samples were taken at $0600 \mathrm{~h}$ and 0930h. Demographics are in Table 3. 
Table 3: Demographics: ROTC cadets

\begin{tabular}{|l|l|l|l|l|l|}
\hline Exercise & \# subjects & \# females & \# males & Age range & Mean \\
\hline 1 & 9 & 2 & 7 & $18-23$ & 19.9 \\
\hline 2 & 6 & 0 & 6 & $18-22$ & 19.7 \\
\hline 3 & 7 & 0 & 7 & $19-22$ & 20.6 \\
\hline
\end{tabular}




\section{Circadian rhythm studies}

Studies were undertaken in order to determine if s-VIP has any circadian or time variation. Student volunteers collected saliva at $0800 \mathrm{~h}, 1200 \mathrm{~h}, 1600 \mathrm{~h}, 2000 \mathrm{~h}, 2400 \mathrm{~h}$ and 0400h. Students were asked to not eat or drink 1 hour prior to sample collection. Demographics are in Table 4a.

The effect of drinking a bottle of Water

Student volunteers collected saliva before and after consuming a 20oz. Aqua Fina water bottle purchased at the cafeteria. Saliva samples were collected before water consumption ( 0 minutes) and 10, 20 and 30 minutes post water consumption. Time of collection was between 1200-1400h. Students were asked to not eat or drink 1 hour prior to sample collection. Demographics are in Table $4 \mathrm{~b}$.

\section{The effect of drinking coffee}

Student volunteers collected saliva before and after consuming a regular medium coffee (Starbucks breakfast blend) no sugar, no milk, purchased on the campus of Seton Hall University. Saliva samples were collected before coffee consumption ( 0 minutes) and 10, 20 and 30 minutes post coffee consumption. Time of collection was between 1200-1400h. Students were asked to not eat or drink 1 hour prior to sample collection. Demographics are in Table 4c. 
Table 4a. Demographics: Circadian rhythm studies

\begin{tabular}{|l|l|l|l|l|}
\hline Group & \# subjects & \# females & \# males & Age range \\
\hline Circadian & 8 & 4 & 4 & $18-40$ \\
\hline
\end{tabular}

Table 4b. Demographics water intake study

\begin{tabular}{|l|l|l|l|l|}
\hline Group & \# subjects & \# females & \# males & Age range \\
\hline Water & 11 & 6 & 5 & $18-40$ \\
\hline
\end{tabular}

Table 4c. Demographics in coffee intake study

\begin{tabular}{|l|l|l|l|l|}
\hline Group & \# subjects & \# females & \# males & Age range \\
\hline Coffee & 11 & 6 & 5 & $18-40$ \\
\hline
\end{tabular}


Salivary sample and assays

Saliva samples were collected into $15 \mathrm{ml}$ polypropylene centrifuge tubes. One tube contained citrate to prevent enzymatic degradation of peptides. Samples were stored at $-70^{\circ} \mathrm{C}$ until assayed. Before the assay, samples were thawed and spun at $3000 \mathrm{rpm}$ or $1,787 \mathrm{~g}$ for 15 minutes. Samples were assayed by ELISA kits obtained from Salimetrics LLC (State College, PA) for cortisol (cat. \# 1-3002) and DHEA (cat \# 1-1202). Salivary amylase (cat \# 1-1902) was measured by enzymatic activity (Salimetrics LLC (State College, PA). Once the analyses are completed, any remaining samples were thrown away. The limit of sensitivity for cortisol, DHEA, and amylase assays are $0.012 \mu \mathrm{g} / \mathrm{dL}$, $10.2 \mathrm{pg} / \mathrm{mL}$ and $10 \mathrm{IU} / \mathrm{mL}$, respectively. CV\% intraassay for Cortisol, DHEA and Amylase were $8.14 \%, 9.45 \%$ and $4.74 \%$ respectively. CV\% interassay for Cortisol, DHEA and Amylase were $15.70 \%, 15.68 \%$ and $14.14 \%$, respectively.

Samples for salivary NPY (cat \# RK-049-03) and VIP (cat \# RK-064-16) were assayed by radioimmunassay (RIA) using kits obtained from Phoenix Pharmaceuticals (Burlingame, CA) (Allen et al., 1991, Hunter et al., 1998). The limit of sensitivity for the peptide RIAs is $10 \mathrm{pg} / \mathrm{mL}$. NPY values in saliva were routinely found to be below $10 \mathrm{pg} / \mathrm{mL}$ therefore spiking the samples in the assay with $10 \mathrm{pg} / \mathrm{ml}$ was used as strategy to put the samples above the limit of detection. The final concentration of the NPY samples was subsequently calculated by subtracting $10 \mathrm{pg} / \mathrm{mL}$. Percent recovery for NPY and VIP were 91.5 and $87.5 \%$, respectively. CV\% intraassay for VIP and NPY were $4.33 \%$ and $3.91 \%$ respectively. CV\% interassay for VIP and NPY were $14.59 \%$ and $11.18 \%$, respectively. 


\section{$N P Y \& V I P \%$ recovery}

Saliva samples were collected in a variety of different substances to prevent degradation of peptides in saliva including Trasylol, EDTA, HCl, HF and citrate. (See Table $9 \mathrm{a} \& 9 \mathrm{~b}$ for details).

Preliminary studies were performed to determine the best method to collect saliva in the field to prevent degradation of the peptides. A widely used cocktail for preserving peptides in blood is EDTA $(2.5 \mathrm{mg} / \mathrm{mL})$ and Trasylol (600KIU). This is the cocktail that was initially used for the initial percent recovery studies. The initial studies used saliva spiked with radioactive NPY. The other mixtures contain different concentrations of $\mathrm{NaCitrate}$ which is used as an anticoagulant in blood and $\mathrm{HCl}$ or TFA. The least loss was achieved with the cocktail which contained $100 \mathrm{mM}$ NaCitrate (Table 5). Surprisingly, $100 \mathrm{mM}$ NaCitrate alone did just as well or better than the mixtures. The VIP data in (Table 6) mirrored the NPY results. Based on this data, the next round of experiments focused on trying to preserve NPY and VIP in saliva samples spiked with cold peptide. The results in (Table 7) clearly show that $100 \mathrm{mM} \mathrm{NaCitrate}$ works as well as the EDTA/Trasylol/NaCitrate/ $\mathrm{HCl}$ cocktail. Percent recovery was at approximately $90 \%$ for both. The results for VIP in (Table 7) produced results of $87.5 \%$ for NaCitrate and $89.1 \%$ for the cocktail. Therefore, the results are consistent for both neuropeptides. 
Table 5. Percent of NPY recovery using different preservative mixtures

\begin{tabular}{|c|c|}
\hline$\underline{\text { mixture }}$ & \% NPY recovery \\
\hline$\underline{\mathbf{1}}$ & $\mathbf{7 3}$ \\
\hline$\underline{\mathbf{2}}$ & $\mathbf{7 6}$ \\
\hline$\underline{\mathbf{3}}$ & $\mathbf{6 8}$ \\
\hline$\underline{\mathbf{4}}$ & $\mathbf{8 0}$ \\
\hline$\underline{\mathbf{5}}$ & $\mathbf{8 0}$ \\
\hline$\underline{\mathbf{6}}$ & $\mathbf{8 3}$ \\
\hline
\end{tabular}

1.) $\operatorname{EDTA}(2.5 \mathrm{mg} / \mathrm{ml})$, Trasylol (600KIU)

2.) EDTA (2.5mg/ml), Trasylol (600KIU), NaCitrate (10mM), HCl (10mM)

3.) EDTA (2.5mg/ml), Trasylol (600KIU), NaCitrate (10mM), TFA (0.1\%)

4.) EDTA (2.5mg/ml), Trasylol (600KIU),NaCitrate (100mM), HCl (10mM)

5.) EDTA (2.5mg/ml), Trasylol (600KIU),NaCitrate (100mM), TFA (0.1\%)

6.) NaCitrate $(100 \mathrm{mM})$ 
Table 6. Percent of VIP recovery using different preservative mixtures

\begin{tabular}{|c|c|}
\hline$\underline{\text { mixture }}$ & \% VIP recovery \\
\hline 1 & 72 \\
\hline 2 & 73 \\
\hline 3 & 91 \\
\hline 4 & 90 \\
\hline 5 & 92 \\
\hline 6 & 89 \\
\hline
\end{tabular}

1.) $\operatorname{EDTA}(2.5 \mathrm{mg} / \mathrm{ml})$, Trasylol (600KIU)

2.) EDTA (2.5mg/ml), Trasylol (6000KIU), $\mathrm{HCl}(10 \mathrm{mM})$

3.) EDTA $(2.5 \mathrm{mg} / \mathrm{ml}), \mathrm{NaCitrate}(100 \mathrm{mM})$

4.) EDTA (2.5mg/ml), Trasylol (600KIU),NaCitrate (100mM), HCl (10mM)

5.) NaCitrate $(150 \mathrm{mM})$

6.) NaCitrate $(100 \mathrm{mM})$ 
Table 7. Preservation and determination of immunoreactive NPY and VIP using saliva samples spiked with known amounts of cold NPY or cold VIP

\begin{tabular}{|c|c|c|}
\hline mixture & \% recovery of NPY & \% recovery of VIP \\
\hline 1 & 91.5 & 87.5 \\
\hline 2 & 90.2 & 89.1 \\
\hline
\end{tabular}

Mixture 1-NaCitrate (100mM)

Mixture 2-EDTA (2.5mg/ml), Trasylol (1000KIU),NaCitrate (100mM), HCl (10mM)

Note: For NPY each \% represents an average of 4 different saliva samples spiked with the following concentrations of cold NPY: 128, 64, 36 \& 25.6 picograms. For VIP each $\%$ represents an average of 2 different saliva samples spiked with the following concentrations of cold VIP: $64 \& 25.6$ picograms 


\section{Solid Phase Extraction}

A method of solid phase extraction was examined in case the levels of peptides in saliva were too low to detect without concentration. Solid phase extraction procedures were tested according to Hunter et al. and Allen et al. (Allen et al., 1991; Hunter et al., 1998) The Hunter extraction was used with a C18 column as the solid phase. It was washed with $2 \mathrm{~mL}$ acetonitrile and rinsed with $10 \mathrm{~mL}$ water. It was conditioned with 2 $\mathrm{mL}$ of $1 \%$ bovine serum albumin (BSA). The sample was applied and then the column was rinsed with $5 \mathrm{~mL}$ water. The peptide was eluted from the column with $2.5 \mathrm{~mL}$ trifluoroacetic acid, acetonitrile (1\%:80\%) and collected into a tube containing $0.1 \mathrm{~mL}$ $0.67 \%$ BSA. Samples were then lyophilized and resuspended in $250 \mu \mathrm{L}$ of RIA buffer (Allen et al., 1991, Hunter et al., 1998) Results for extraction of NPY and VIP from saliva using the Hunter method are shown in Table 8 . Ninety $\%$ recovery was found for NPY and $68 \%$ for VIP. These results are similar to the Hunter paper which achieved 89.5 and $77.4 \%$ recovery for NPY and VIP, respectively. 
Table 8. Recovery of radioactive NPY and VIP in saliva after solid phase extraction \% recovery of NPY $\quad$ \% recovery of VIP 90 68 


\section{Statistical analysis}

Statistical analysis was performed using Number cruncher statistical systems (NCSS) (Hintze, 1998). Repeated measure ANOVA followed by a Tukey-Kramer Multiple-Comparison Test to determine if a specific time point was statistically significant from the others was used. If the study consisted of only two groups, a paired t-test was used in lieu of the rmANOVA followed by Tukey-Kramer. Physiological parameters (salivary cortisol, DHEA, amylase, NPY, VIP) were correlated with each other and the questionnaire data using linear regression. 


\section{Results}

\section{Disaster drill: PAD}

The disaster drill study assessed the stress the participants were experiencing before and after the training was over. The statistics used was the Students two tailed paired T-Test. Salivary markers did not demonstrate any increase. On the contrary, salivary cortisol $(\mathrm{p}<0.001)$ and DHEA $(\mathrm{p}=0.036)$ (Fig. 4a) levels decreased significantly probably due to their circadian rhythms. Salivary samples were collected before the initiation of the training exercise approximately $0700 \mathrm{~h}$, and after completion of the drills, which was early afternoon (1300 h). Salivary amylase levels, a possible marker for sympathetic activation did not change $(\mathrm{p}=0.212)$ (Fig. 4a). The salivary neuropeptides VIP ( $\mathrm{p}=0.210)$ (Fig. 4b) and NPY ( $\mathrm{p}=0.181)$ (Fig. 4c) showed no significant difference. Taken together, the data suggests the PAD training exercise did not cause any increase in stress markers at the times taken.

The following linear regressions were found to be significant and positive relationships: Cortisol vs. DHEA $\left[\mathrm{R}^{2}=0.6225, \mathrm{~F}(1,13)=19.79, \mathrm{p}=0.0008\right]$; VIP vs. NPY $\left[\mathrm{R}^{2}=0.7480, \mathrm{~F}(1,13)=35.61, \mathrm{p}<0.0001\right] ;$ DHEA vs. NPY $\left[\mathrm{R}^{2}=0.3822, \mathrm{~F}(1,13)=7.42\right.$,

$\mathrm{p}=0.0184]$; generally feel vs. amylase $\left[\mathrm{R}^{2}=0.4503, \mathrm{~F}(1,11)=8.19, \mathrm{p}=0.0169\right]$. 

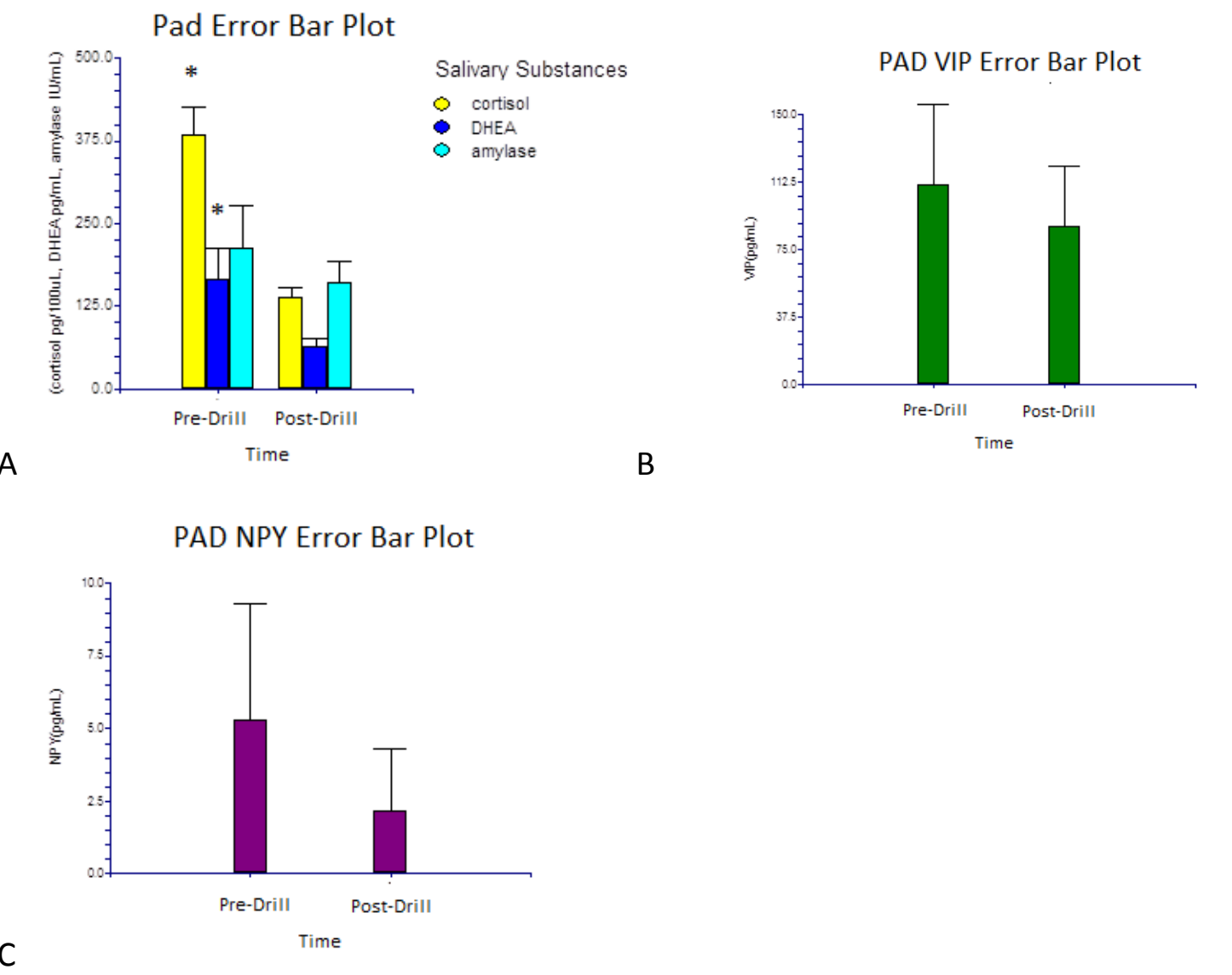

Fig. 4 Changes in salivary levels: Graph A: cortisol, DHEA \& amylase, Graph B-: VIP, Graph C: NPY. Values are depicted as mean $+/-$ standard error. Statistical significances were determined by Students two tailed paired T-Test. 


\section{E1 Exam Group}

The times of collection for the before times were around $0830 \mathrm{~h}$ and the after times were collected approximately at $1200 \mathrm{~h}$, therefore, a circadian effect could be a factor for both s-cortisol and s-DHEA. Using rmANOVA, there was a significant main effect of time of day for s-cortisol, $[\mathrm{F}(7,31)=11.67, \mathrm{p}=0.011]$. The Tukey-Kraemer Multiple Comparison Test reports significant differences for the means for s-cortisol between precontrol and post-control, pre-control and post-exam, and between pre-exam and post control, and pre-exam and post-exam. The significant decrease in salivary cortisol levels in the post-samples is probably due to cortisol's circadian pattern (Fig. 5a). Salivary DHEA levels also showed a main effect of time of day, $[F(7,31)=10.69, p=0.014]$ (Figure 5a). The Tukey-Kraemer Multiple Comparison Test reports significant differences for the means for s-DHEA between pre-control and post-control. The decreases observed in the post-samples for DHEA were probably due to its circadian pattern. For s-amylase, rmANOVA showed a significant main effect of time, $[\mathrm{F}(7,31)=30.47, \mathrm{p}<0.001]$ and a significant main effect among the groups (exam/control), $[F(7,31)=13.20, \mathrm{p}<0.001]$ (Fig. 5a). The Tukey-Kraemer Multiple Comparison Test reports significant differences for samylase between post-exam and post-control, and between post exam and pre-exam. RmANOVA reports no significant main effects for s-VIP (Fig. 5b). For s-NPY, rmANOVA reports a significant interaction between time of day and exam/control groups, $[\mathrm{F}(7,31)=5.82, \mathrm{p}=0.047]$. Tukey-Kraemer reports no significant differences among groups (Fig. 5c). All rmANOVA reports for this study are in Table 9. Tension data for exam data is in Table 14. 
Linear regression analysis among all groups produced significant regressions which were positive relationships for: cortisol vs. DHEA $\left[\mathrm{R}^{2}=0.3991, \mathrm{~F}(1,31)=19.92\right.$, $\mathrm{p}=0.0001]$; and cortisol vs. VIP $\left[\mathrm{R}^{2}=0.2007, \mathrm{~F}(1,31)=7.53, \mathrm{p}=0.0101\right]$; NPY vs. VIP $\left[\mathrm{R}^{2}=0.2097, \mathrm{~F}(1,31)=20.97, \mathrm{p}=0.0084\right]$. 


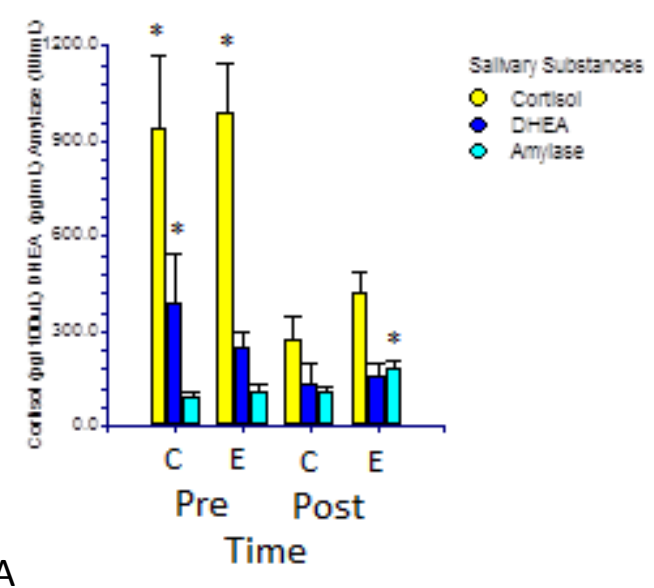

A
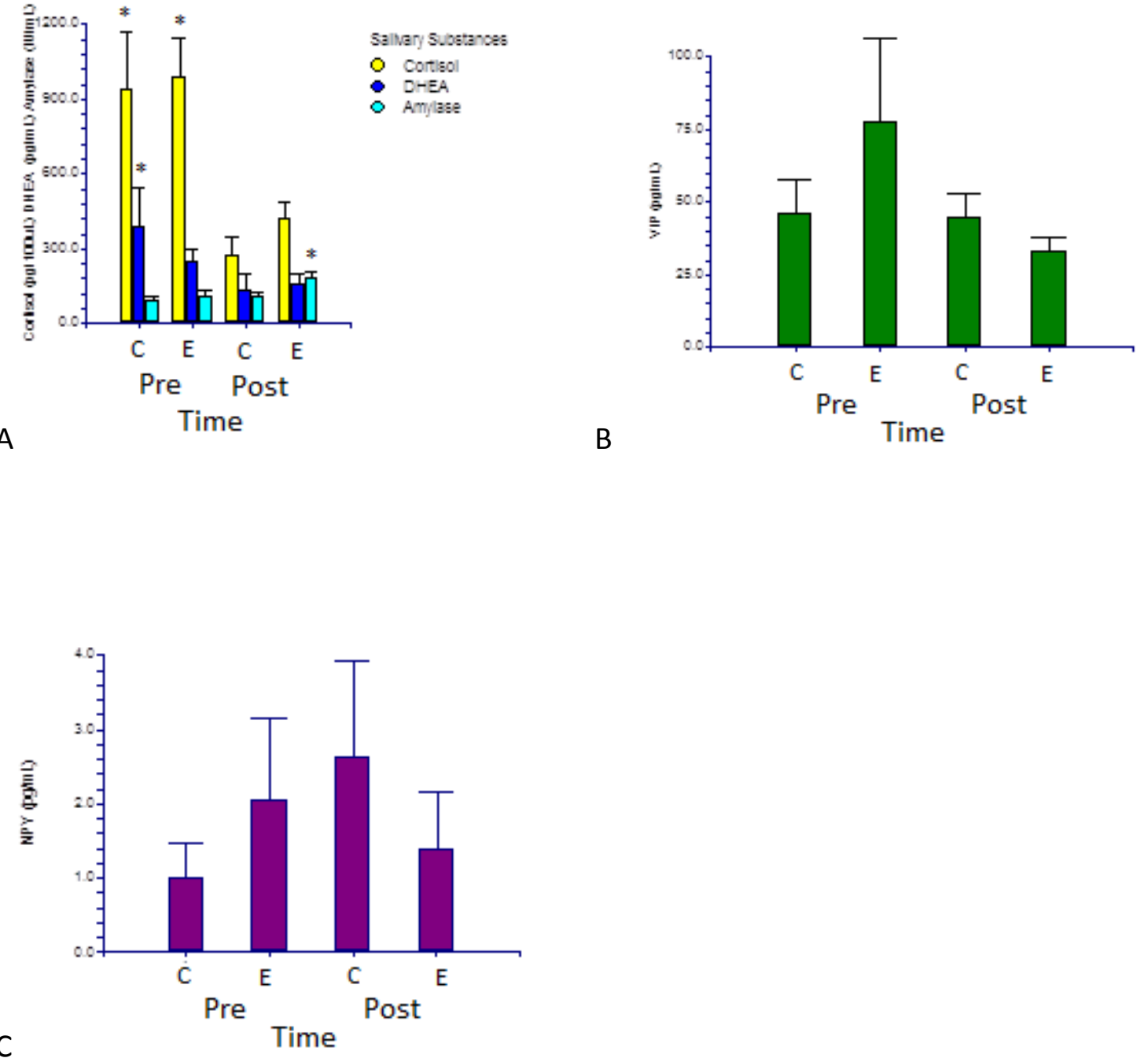

Fig. 5 E1 Exam Group: Changes in salivary levels: Graph A: cortisol, DHEA \& amylase, Graph B-: VIP, Graph C: NPY. Values are depicted as mean +/- standard error.

Statistical significances were determined by repeated measures ANOVA followed by Tukey-Kraemer Multiple-Comparison Test. Asterisks indicate samples that were significantly higher than other samples measuring the same parameter, $\mathrm{p} \leq 0.05$. Other significant effects are shown in Table 9. 
Table 9 E1 Exam Group: Results of Repeated Measures ANOVA: Effect of Time of day, Effect of Exam/Control groups, Interaction between Time of day and Exam/Control.

\begin{tabular}{|l|l|l|}
\hline Cortisol & Time of day & {$[\mathrm{F}(7,31)=11.67, \mathrm{p}=0.011]$} \\
\hline Cortisol & Exam/Control & {$[\mathrm{F}(7,31)=0.75, \mathrm{p}=0.417]$} \\
\hline Cortisol & Time of day $x$ Exam/Control & {$[\mathrm{F}(7,31)=0.58, \mathrm{p}=0.469]$} \\
\hline DHEA & Time of day & {$[\mathrm{F}(7,31)=10.69, \mathrm{p}=0.014]$} \\
\hline DHEA & Exam/Control & {$[\mathrm{F}(7,31)=0.48, \mathrm{p}=0.510]$} \\
\hline DHEA & Time of day $x$ Exam/Control & {$[\mathrm{F}(7,31)=2.60, \mathrm{p}=0.151]$} \\
\hline Amylase & Time of day & {$[\mathrm{F}(7,31)=30.47, \mathrm{p}<0.001$} \\
\hline Amylase & Exam/Control & {$[\mathrm{F}(7,31)=13.20, \mathrm{p}<0.001$} \\
\hline Amylase & Time of day x Exam/Control & {$[\mathrm{F}(7,31)=5.14, \mathrm{p}=0.058$} \\
\hline VIP & Time of day & {$[\mathrm{F}(7,31)=2.10, \mathrm{p}=0.191$} \\
\hline VIP & Exam/Control & {$[\mathrm{F}(7,31)=1.29, \mathrm{p}=0.293$} \\
\hline VIP & Time of day $\mathrm{x}$ Exam/Control & {$[\mathrm{F}(7,31)=2.57, \mathrm{p}=0.153$} \\
\hline NPY & Time of day & {$[\mathrm{F}(7,31)=0.40, \mathrm{p}=0.547$} \\
\hline NPY & Exam/Control & {$[\mathrm{F}(7,31)=0.01, \mathrm{p}=0.927$} \\
\hline NPY & Time of day $x$ Exam/Control & {$[\mathrm{F}(7,31)=5.82, \mathrm{p}=0.047$} \\
\hline
\end{tabular}




\section{E2 Exam Group}

The times of collection for before times were around 1300h and the after times were collected approximately at 1430h. Using rmANOVA, there was a significant main effect of time of day for s-cortisol, $[\mathrm{F}(9,39)=10.61, \mathrm{p}<0.01]$. The Tukey-Kraemer Multiple Comparison Test reports significant differences for the means for s-cortisol between pre-control and post-control, pre-control and post-exam and between pre-exam and post-control and pre-exam and post-exam. The significant decrease in s-cortisol levels in the post-samples is probably due to cortisol's circadian pattern (Fig. 6a).

Salivary DHEA levels also showed a main effect of time of day, $[F(9,39)=7.79, p=0.021]$ (Fig. 6a). The Tukey-Kraemer Multiple Comparison Test reports significant differences for the means for s-DHEA between pre-control and post-control. The decreases observed in the post-samples for s-DHEA are probably due to its circadian pattern. For s-amylase, rmANOVA showed a significant main effect for time of day, $[F(9,39)=6.49, p=0.031]$. The Tukey-Kraemer Multiple Comparison Test reports no significant differences of the means for s-amylase (Fig. 6a). RmANOVA reports a significant main effect of time of day for s-VIP, $[\mathrm{F}(9,39)=5.26, \mathrm{p}=0.047]$. The Tukey-Kraemer Multiple Comparison Test reports no significant differences the means for s-VIP (Fig.6b). Using rmANOVA, there was a significant main effect of time of day for $s-\mathrm{NPY},[\mathrm{F}(5,23)=8.35, \mathrm{p}=0.034]$ (Fig. 6c). The Tukey-Kraemer Multiple Comparison Test reports significant differences for the means for s-NPY between post-control and pre-control, and post-control and pre-exam. All rmANOVA reports for this study are in Table 10. Tension data for exam data is in Table 14. 
Linear regression analysis for all groups produced the following significant regression which were positive relationships for: cortisol vs. DHEA $\left[\mathrm{R}^{2}=0.1773\right.$, $F(1,39)=8.191, p=0.0068] ;$ VIP vs. NPY $\left[R^{2}=0.4457, F(1,25)=19.297, p=0.0002\right]$; amylase vs. VIP $\left[R^{2}=0.1447, F(1,38)=6.258, p=0.0169\right]$. negative relationships for: VIP vs. tense arousal $\left[\mathrm{R}^{2}=0.1103, \mathrm{~F}(1,39)=4.709, \mathrm{p}=0.0363\right]$; VIP vs. tension $\left[\mathrm{R}^{2}=0.1023\right.$, $\mathrm{F}(1,38)=4.218, \mathrm{p}=0.0471]$. 


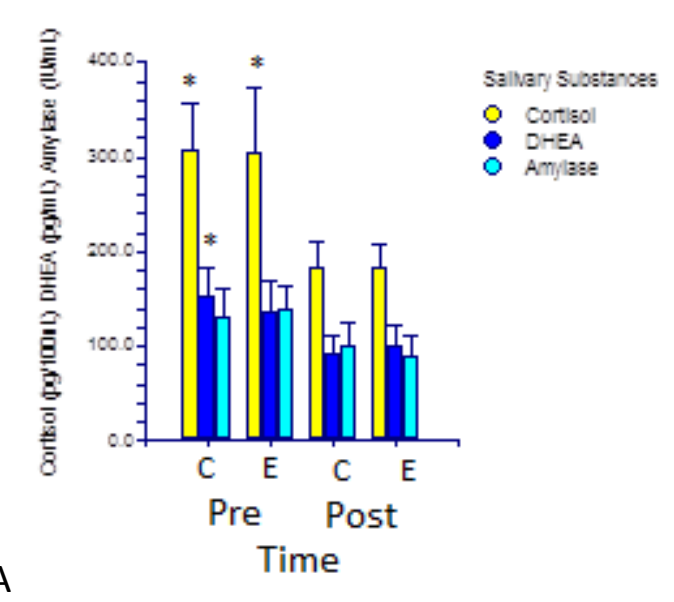

A

B
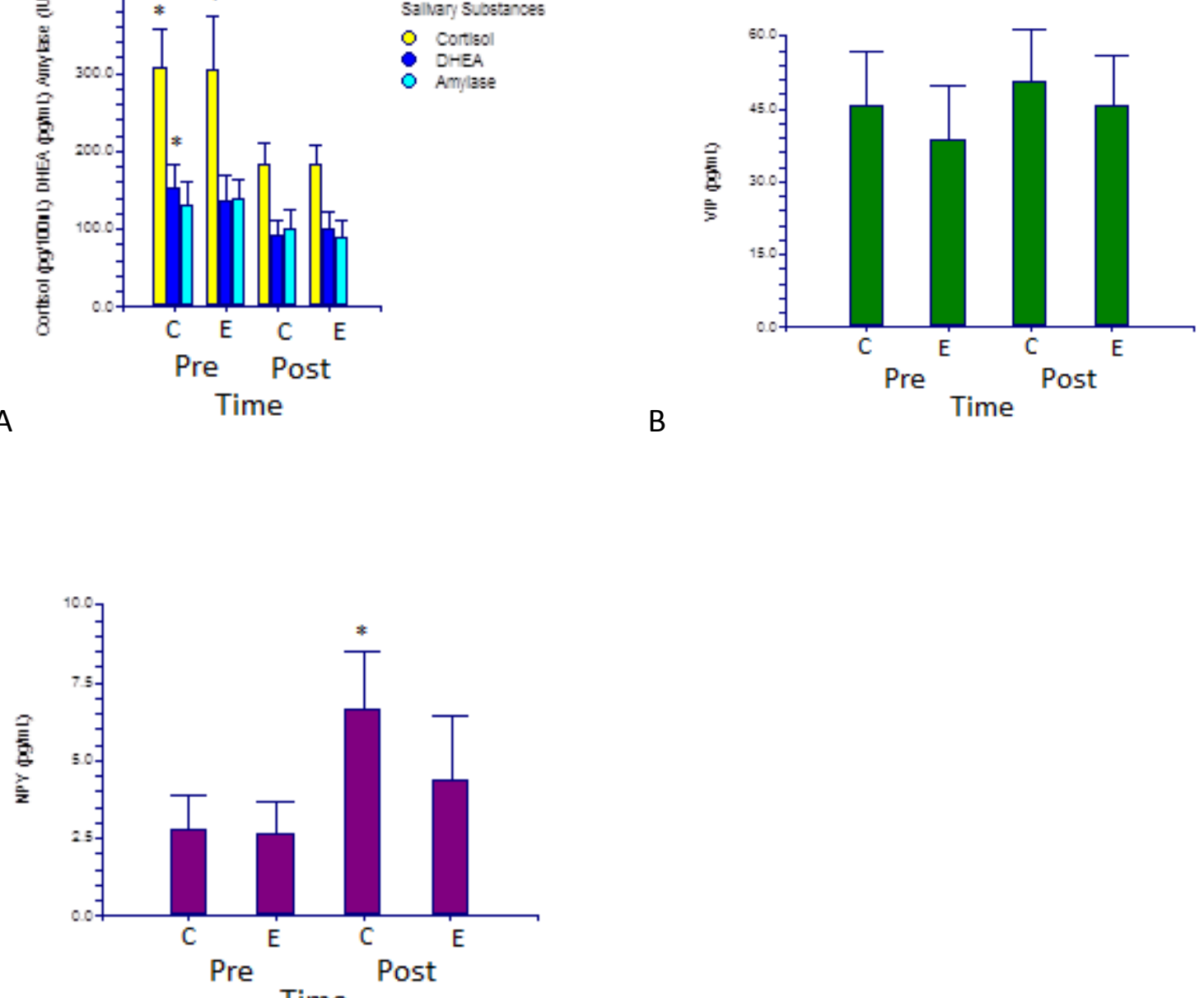

C

Fig. 6 E2 Exam Group: Changes in salivary levels: Graph A: cortisol, DHEA \& amylase, Graph B-: VIP, Graph C: NPY. Values are depicted as mean +/- standard error. Statistical significances were determined by repeated measures ANOVA followed by Tukey-Kraemer Multiple-Comparison Test. Asterisks indicate samples that were significantly higher than other samples measuring the same parameter, $\mathrm{p} \leq 0.05$. Other significant effects are shown in Table 10. 
Table 10 E2 Exam Group: Results of Repeated Measures ANOVA: Effect of Time of day, Effect of Exam/Control groups, Interaction between Time of day and Exam/Control.

\begin{tabular}{|l|l|l|}
\hline Cortisol & Time of day & {$[\mathrm{F}(9,39)=10.61, \mathrm{p}<0.01]$} \\
\hline Cortisol & Exam/Control & {$[\mathrm{F}(9,39)=0.00, \mathrm{p}=0.975]$} \\
\hline Cortisol & Time of day $x$ Exam/Control & {$[\mathrm{F}(9,39)=0.00, \mathrm{p}=0.980]$} \\
\hline DHEA & Time of day & {$[\mathrm{F}(9,39)=7.79, \mathrm{p}=0.021]$} \\
\hline DHEA & Exam/Control & {$[\mathrm{F}(9,39)=0.02, \mathrm{p}=0.892]$} \\
\hline DHEA & Time of day $\mathrm{x}$ Exam/Control & {$[\mathrm{F}(9,39)=1.15, \mathrm{p}=0.312]$} \\
\hline Amylase & Time of day & {$[\mathrm{F}(9,39)=6.49, \mathrm{p}=0.031]$} \\
\hline Amylase & Exam/Control & {$[\mathrm{F}(9,39)=0.14, \mathrm{p}=0.716]$} \\
\hline Amylase & Time of day x Exam/Control & {$[\mathrm{F}(9,39)=0.01, \mathrm{p}=0.913]$} \\
\hline VIP & Time of day & {$[\mathrm{F}(9,39)=5.26, \mathrm{p}=0.047]$} \\
\hline VIP & Exam/Control & {$[\mathrm{F}(9,39)=0.29, \mathrm{p}=0.604]$} \\
\hline VIP & Time of day $\mathrm{x}$ Exam/Control & {$[\mathrm{F}(9,39)=0.03, \mathrm{p}=0.871]$} \\
\hline NPY & Time of day & {$[\mathrm{F}(5,23)=8.35, \mathrm{p}=0.034]$} \\
\hline NPY & Exam/Control & {$[\mathrm{F}(5,23)=0.48, \mathrm{p}=0.519]$} \\
\hline NPY & Time of day $x$ Exam/Control & {$[\mathrm{F}(5,23)=3.27, \mathrm{p}=0.130]$} \\
\hline
\end{tabular}




\section{E3 Exam Group}

The times of collection for before times were around 1300h and the after times were collected approximately at 1430h. Using rmANOVA, the E3 Exam group showed no significant main effects for time of day and exam/control groups for s-cortisol, sDHEA and s-amylase (Fig. 7a). Tukey-Kraemer did not report any differences of the means for s-cortisol, s-DHEA and s-amylase. Also, there was no significant interaction between time of day and exam/control groups for s-cortisol, s-DHEA and s-amylase. RmANOVA reports a significant interaction between time of day and exam/control groups, $[\mathrm{F}(4,19)=16.56, \mathrm{p}=0.015]$ for $\mathrm{s}-\mathrm{VIP}$ (Fig. 7b). The Tukey-Kraemer Multiple Comparison Test reports significant differences for the means for s-VIP between precontrol and post-control. RmANOVA reports a significant main effect among the exam/control groups for s-NPY, [F(4,19)=23.44, p<0.01] (Fig. 7c). The Tukey-Kraemer Multiple Comparison Test reports significant differences for the means for s-NPY between post-control and post-exam. All rmANOVA reports for this study are in Table 11. Tension data for exam data is in Table 14.

Linear regression analysis for all groups produced the following significant regressions which were positive relationships for: Cortisol vs. DHEA $\left[R^{2}=0.6593\right.$, $\mathrm{F}(1,19)=34.835, \mathrm{p}<0.0001] ;$; cortisol vs. amylase $\left[\mathrm{R}^{2}=0.2278, \mathrm{~F}(1,19)=5.309, \mathrm{p}=0.0333\right]$; amylase vs. NPY $\left[\mathrm{R}^{2}=0.2238, \mathrm{~F}(1,19)=5.190, \mathrm{p}=0.0351\right]$; negative relationships for: cortisol vs. VIP $\left[\mathrm{R}^{2}=0.263, \mathrm{~F}(1,19)=6.433, \mathrm{p}=0.0207\right]$; DHEA vs. VIP $\left[\mathrm{R}^{2}=0.2807\right.$, $\mathrm{F}(1,19)=7.025, \mathrm{p}=0.0163]$. 


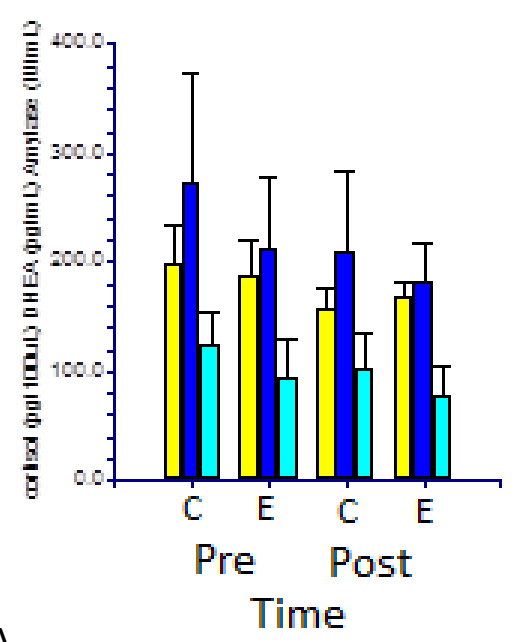

A

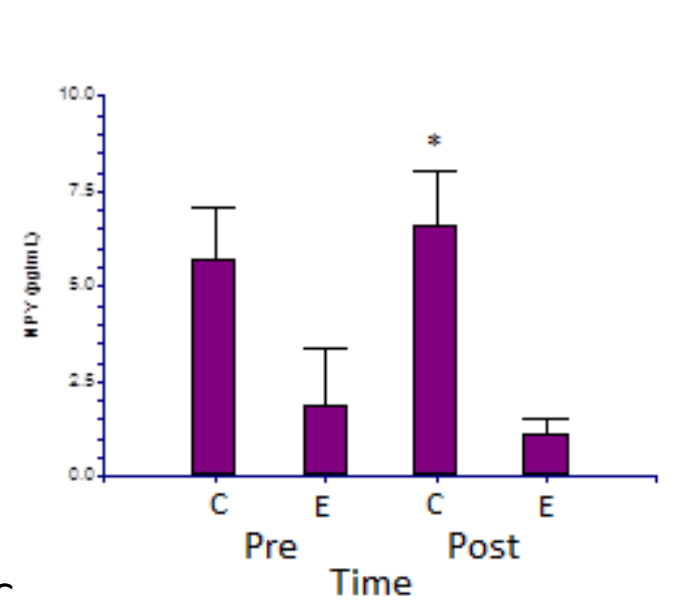

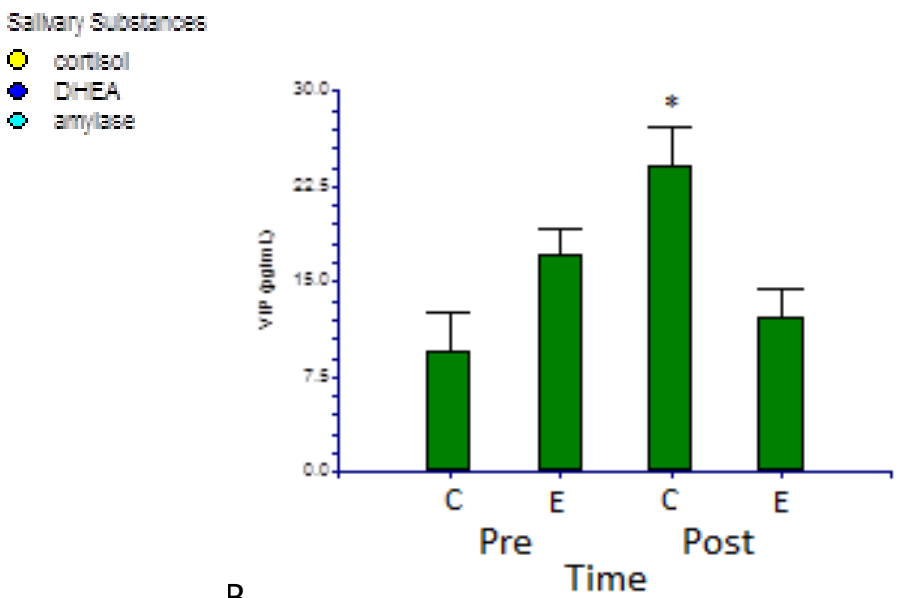

B

C

Fig. 7 E3 Exam Group: Changes in salivary levels: Graph A: cortisol, DHEA \& amylase, Graph B-: VIP, Graph C: NPY. Values are depicted as mean +/- standard error.

Statistical significances were determined by repeated measures ANOVA followed by Tukey-Kraemer Multiple-Comparison Test. Asterisks indicate samples that were significantly higher than other samples measuring the same parameter, $p \leq 0.05$. Other significant effects are shown in Table 11. 
Table 11 E3 Exam Group: Results of Repeated Measures ANOVA: Effect of Time of day, Effect of Exam/Control groups, Interaction between Time of day and Exam/Control.

\begin{tabular}{|l|l|l|}
\hline Cortisol & Time of day & {$[\mathrm{F}(4,19)=2.04, \mathrm{p}=0.226]$} \\
\hline Cortisol & Exam/Control & {$[\mathrm{F}(4,19)=0.00, \mathrm{p}=0.961]$} \\
\hline Cortisol & Time of day $x$ Exam/Control & {$[\mathrm{F}(4,19)=0.30, \mathrm{p}=0.611]$} \\
\hline DHEA & Time of day & {$[\mathrm{F}(4,19)=0.99, \mathrm{p}=0.376]$} \\
\hline DHEA & Exam/Control & {$[\mathrm{F}(4,19)=0.86, \mathrm{p}=0.406]$} \\
\hline DHEA & Time of day $x$ Exam/Control & {$[\mathrm{F}(4,19)=0.67, \mathrm{p}=0.459]$} \\
\hline Amylase & Time of day & {$[\mathrm{F}(4,19)=4.99, \mathrm{p}=0.089]$} \\
\hline Amylase & Exam/Control & {$[\mathrm{F}(4,19)=3.41, \mathrm{p}=0.138]$} \\
\hline Amylase & Time of day x Exam/Control & {$[\mathrm{F}(4,19)=0.02, \mathrm{p}=0.908]$} \\
\hline VIP & Time of day & {$[\mathrm{F}(4,19)=7.62, \mathrm{p}=0.051]$} \\
\hline VIP & Exam/Control & {$[\mathrm{F}(4,19)=4.16, \mathrm{p}=0.111]$} \\
\hline VIP & Time of day $x$ Exam/Control & {$[\mathrm{F}(4,19)=16.56, \mathrm{p}=0.015]$} \\
\hline NPY & Time of day & {$[\mathrm{F}(4,19)=0.01, \mathrm{p}=0.930]$} \\
\hline NPY & Exam/Control & {$[\mathrm{F}(4,19)=23.44, \mathrm{p}<0.01]$} \\
\hline NPY & Time of day $x$ Exam/Control & {$[\mathrm{F}(4,19)=0.81, \mathrm{p}=0.420]$} \\
\hline
\end{tabular}




\section{E4 Exam Group}

The times of collection for the before times were $1730 \mathrm{~h}$ and the after times were approximately at $1900 \mathrm{~h}$. Using rmANOVA, there was a significant main effect of time of day for cortisol, $[\mathrm{F}(3,15)=34.16, \mathrm{p}<0.01]$ (Fig. 8a). Using rmANOVA, the E4 Exam group showed no significant main effects for time of day and exam/control groups for sDHEA and s-amylase (Fig. 8a) and s-VIP (Fig. 8b). Also there was no significant interaction between time of day and exam/control groups for s-cortisol, s-DHEA, samylase, s-VIP and s-NPY. Tukey-Kraemer did not report any differences of the means for s-cortisol, s-DHEA, s-amylase (Fig. 8a), s-VIP (Fig. 8b) and s-NPY (Fig. 8c). All rmANOVA reports for this study are in Table 12. Tension data for exam data is in Table 14.

Linear regression analysis using all groups produced the following significant regressions which were positive relationships for: DHEA vs. cortisol $\left[\mathrm{R}^{2}=0.2908\right.$, $\mathrm{F}(1,15)=5.74, \mathrm{p}=0.0311]$; atm vs. cortisol $\left[\mathrm{R}^{2}=0.2716, \mathrm{~F}(1,15)=5.23, \mathrm{p}=0.0383\right]$; atm vs. DHEA $\left.\left[\mathrm{R}^{2}=0.4657, \mathrm{~F}(1,15)=12.20\right], \mathrm{p}=0.0036\right]$ negative relationship for: atm vs. VIP $\left[\mathrm{R}^{2}=0.3494, \mathrm{~F}(1,15)=7.51, \mathrm{p}=0.0159\right]$. 

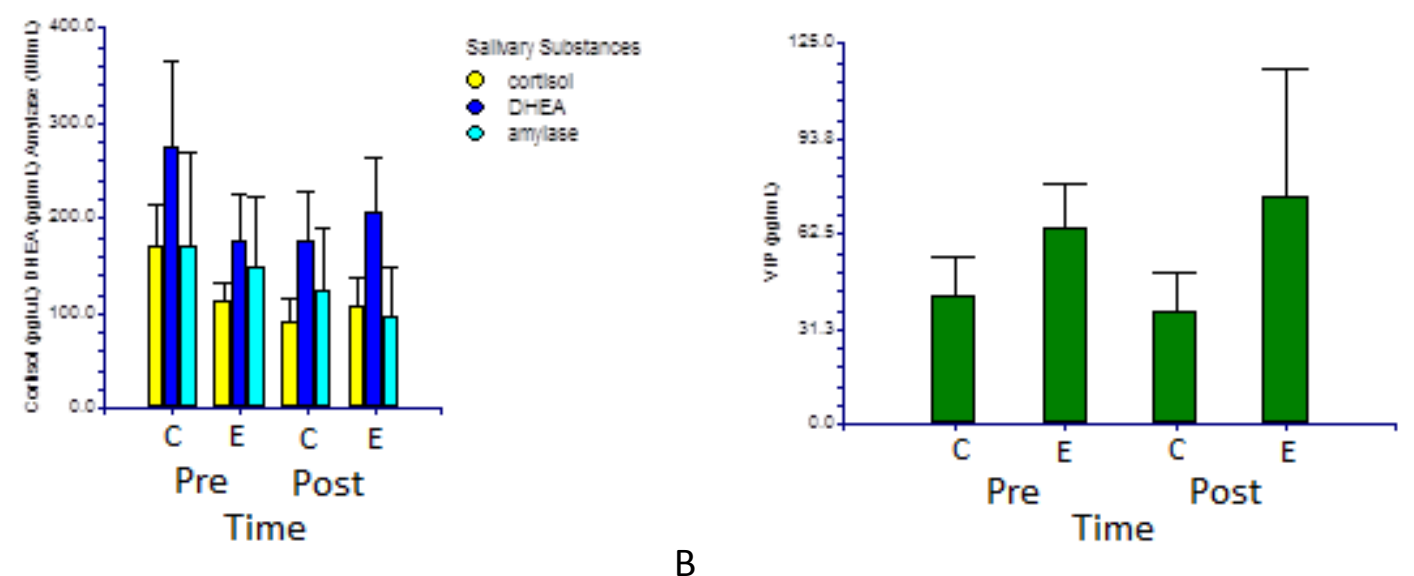

B

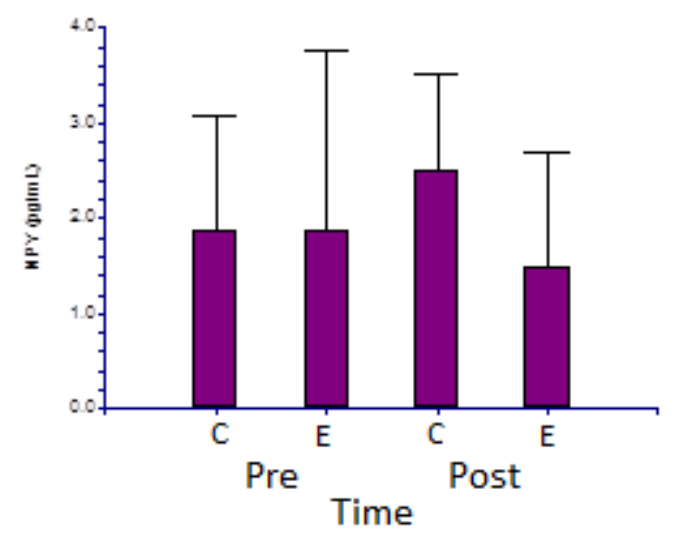

C

Fig. 8 E4 Exam Group: Changes in salivary levels: Graph A: cortisol, DHEA \& amylase, Graph B-: VIP, Graph C: NPY. Values are depicted as mean +/- standard error.

Statistical significances were determined by repeated measures ANOVA followed by Tukey-Kraemer Multiple-Comparison Test. Asterisks indicate samples that were significantly higher than other samples measuring the same parameter, $p \leq 0.05$. Other significant effects are shown in Table 12. 
Table 12 E4 Exam Group: Results of Repeated Measures ANOVA: Effect of Time of day, Effect of Exam/Control groups, Interaction between Time of day and Exam/Control.

\begin{tabular}{|l|l|l|}
\hline Cortisol & Time of day & {$[\mathrm{F}(3,15)=34.16, \mathrm{p}<0.01]$} \\
\hline Cortisol & Exam/Control & {$[\mathrm{F}(3,15)=2.10, \mathrm{p}=0.243]$} \\
\hline Cortisol & Time of day $x$ Exam/Control & {$[\mathrm{F}(3,15)=5.40, \mathrm{p}=0.103]$} \\
\hline DHEA & Time of day & {$[\mathrm{F}(3,15)=1.55, \mathrm{p}=0.301]$} \\
\hline DHEA & Exam/Control & {$[\mathrm{F}(3,15)=0.50, \mathrm{p}=0.532]$} \\
\hline DHEA & Time of day $\mathrm{x}$ Exam/Control & {$[\mathrm{F}(3,15)=5.75, \mathrm{p}=0.096]$} \\
\hline Amylase & Time of day & {$[\mathrm{F}(3,15)=0.88, \mathrm{p}=0.418]$} \\
\hline Amylase & Exam/Control & {$[\mathrm{F}(3,15)=0.12, \mathrm{p}=0.750]$} \\
\hline Amylase & Time of day $\mathrm{x}$ Exam/Control & {$[\mathrm{F}(3,15)=0.00, \mathrm{p}=0.969]$} \\
\hline VIP & Time of day & {$[\mathrm{F}(3,15)=0.02, \mathrm{p}=0.895]$} \\
\hline VIP & Exam/Control & {$[\mathrm{F}(3,15)=2.05, \mathrm{p}=0.247]$} \\
\hline VIP & Time of day $\mathrm{x}$ Exam/Control & {$[\mathrm{F}(3,15)=0.24, \mathrm{p}=0.661]$} \\
\hline NPY & Time of day & {$[\mathrm{F}(3,15)=0.00, \mathrm{p}=0.961]$} \\
\hline NPY & Exam/Control & {$[\mathrm{F}(3,15)=0.41, \mathrm{p}=0.569]$} \\
\hline NPY & Time of day $x$ Exam/Control & {$[\mathrm{F}(3,15)=2.67, \mathrm{p}=0.200]$} \\
\hline
\end{tabular}

E5 Exam Group 
The times of collection for before times were around $1500 \mathrm{~h}$ and the after times were collected approximately at $1700 \mathrm{~h}$. Using rmANOVA, there was a significant main effect of time of day for cortisol, $[F(7,31)=7.75, p=0.027]$ (Fig. 9a). The Tukey-Kraemer Multiple Comparison Test reports significant differences for the means for cortisol between pre-exam and post-control. Salivary DHEA levels showed a significant main effect for exam/control groups $[\mathrm{F}(7,31)=8.49, \mathrm{p}=0.023]$ (Fig. 9a). The Tukey-Kraemer Multiple Comparison Test reports no significant differences for the individual means for s-DHEA. For salivary amylase, there was a significant main effect for exam/control groups, $[\mathrm{F}(3,31)=12.29, \mathrm{p}<0.01]$ (Fig. 9a). The Tukey-Kraemer Multiple Comparison Test reports significant differences for the means for s-amylase between pre-exam and pre-control and pre-exam and post control levels. For s-VIP, rmANOVA did not report any significant differences for a main effect for time of day and exam/control groups. Likewise there was no significant interaction between time of day and exam/control groups (Fig. 9b). The Tukey-Kraemer comparison test for differences among the means reports differences between pre-exam and pre-control, pre-exam and post-control, postexam and pre-control, post-exam and post-control. However, post hoc analysis is performed only when there are significant main effects. All rmANOVA reports for this study are in Table 13. Tension data for exam data is in Table 14.

Linear regression analysis produced significant regressions which were positive relationships for: cortisol vs. DHEA $\left[\mathrm{R}^{2}=0.2704, \mathrm{~F}(1,31)=11.12, \mathrm{p}=0.0023\right]$; amylase vs. VIP $\left[\mathrm{R}^{2}=0.1517, \mathrm{~F}(1,31)=5.36, \mathrm{p}=0.028\right]$. 


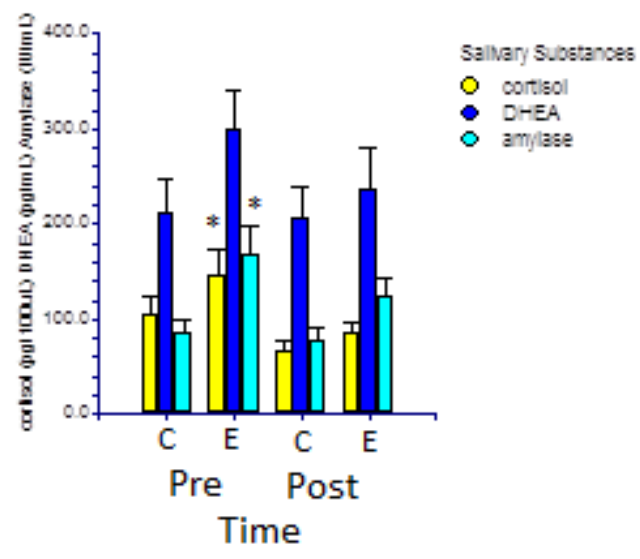

A

Time

B

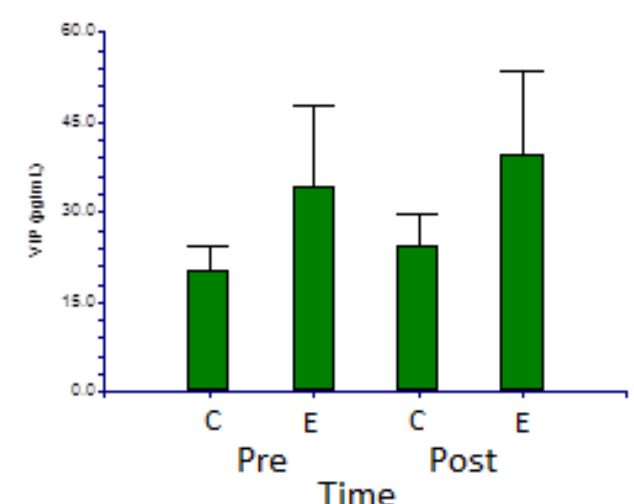

Fig. 9 E5 Exam Group: Changes in salivary levels: Graph A: cortisol, DHEA \& amylase, Graph B-: VIP, Graph C: NPY. Values are depicted as mean +/- standard error. Statistical significances were determined by repeated measures ANOVA followed by Tukey-Kraemer Multiple-Comparison Test. Asterisks indicate samples that were significantly higher than other samples measuring the same parameter, $p \leq 0.05$. Other significant effects are shown in Table 13.

Table 13 E5 Exam Group: Results of Repeated Measures ANOVA: Effect of Time of day, Effect of Exam/Control groups, Interaction between Time of day and Exam/Control. 


\begin{tabular}{|l|l|l|}
\hline Cortisol & Time of day & {$[\mathrm{F}(7,31)=7.75, \mathrm{p}=0.027]$} \\
\hline Cortisol & Exam/Control & {$[\mathrm{F}(7,31)=3.16, \mathrm{p}=0.119]$} \\
\hline Cortisol & Time of day $x$ Exam/Control & {$[\mathrm{F}(7,31)=0.61, \mathrm{p}=0.459]$} \\
\hline DHEA & Time of day & {$[\mathrm{F}(7,31)=1.79, \mathrm{p}=0.223]$} \\
\hline DHEA & Exam/Control & {$[\mathrm{F}(7,31)=8.49, \mathrm{p}=0.023]$} \\
\hline DHEA & Time of day $x$ Exam/Control & {$[\mathrm{F}(7,31)=1.32, \mathrm{p}=0.288]$} \\
\hline Amylase & Time of day & {$[\mathrm{F}(7,31)=2.79, \mathrm{p}=0.139]$} \\
\hline Amylase & Exam/Control & {$[\mathrm{F}(7,31)=12.29, \mathrm{p}<0.01]$} \\
\hline Amylase & Time of day $\mathrm{x}$ Exam/Control & {$[\mathrm{F}(7,31)=1.08, \mathrm{p}=0.334]$} \\
\hline VIP & Time of day & {$[\mathrm{F}(7,31)=2.43, \mathrm{p}=0.163]$} \\
\hline VIP & Exam/Control & {$[\mathrm{F}(7,31)=1.23, \mathrm{p}=0.305]$} \\
\hline VIP & Time of day $\mathrm{x}$ Exam/Control & {$[\mathrm{F}(7,31)=0.10, \mathrm{p}=0.760]$} \\
\hline
\end{tabular}


Table 14 Results: Exam Stress: tension: rmANOVA:means

\begin{tabular}{|l|l|l|l|l|}
\hline & Pre & Pre & Post & Post \\
\hline Group & Control & Exam & Control & Exam \\
\hline E1 exam group & 8.9 & 14.0 & 9.7 & 10.9 \\
\hline E2 Exam group & 7.6 & $13.3,{ }^{*}$ & 8.9 & 8.6 \\
\hline E3 Exam group & 8.8 & 14.6 * $^{*}$ & 8.4 & 12.4 \\
\hline E4 Exam group & 7.8 & 10.0 & 7.5 & 8.5 \\
\hline E5 Exam group & 7.3 & $13.6, *$ & 7.6 & 8.3 \\
\hline
\end{tabular}

*, pre-exam is different from post-exam, pre-control \& post-control

Using Thayer's Activation-Deactivation Checklist (ADCL) tension was analyzed for a main effect for time using rmANOVA followed by Tukey-Kramer multiple comparison test. The results are as follows: E1 exam group: $[\mathrm{F}(3,27)=3.0 \mathrm{p}=0.0579]$; E2 Exam group: $[\mathrm{F}(3,31)=11.3 \mathrm{p}=0.000127]$; E3 Exam group: $[\mathrm{F}(3,19)=3.73 \mathrm{p}=0.042]$; E4 Exam group $[\mathrm{F}(3,15)=1.08 \mathrm{p}=0.404]$; E5 Exam group: $[\mathrm{F}(3,31)=16.05 \mathrm{p}=0.000012]$ 


\section{Training Exercise 1}

The statistics used was the Students two tailed paired T-Test. As can be seen in (Fig. 10a), significant increases were observed in salivary cortisol ( $\mathrm{p}=0.016)$, DHEA $(p=0.026)$ and amylase $(p=0.00009)$ levels after the obstacle course as compared to before the obstacle course (Fig. 10a). Significant increases were also detected in salivary VIP ( $\mathrm{p}=0.018)$ levels, although there was an increase in salivary NPY levels after the obstacle course, the standard error was very large and thus the increase was not significant (Fig. 10b). There was a significant increase in the Borg $(\mathrm{p}=0.003)$ (Table 18). Significant Linear regressions are located in Table 15. 

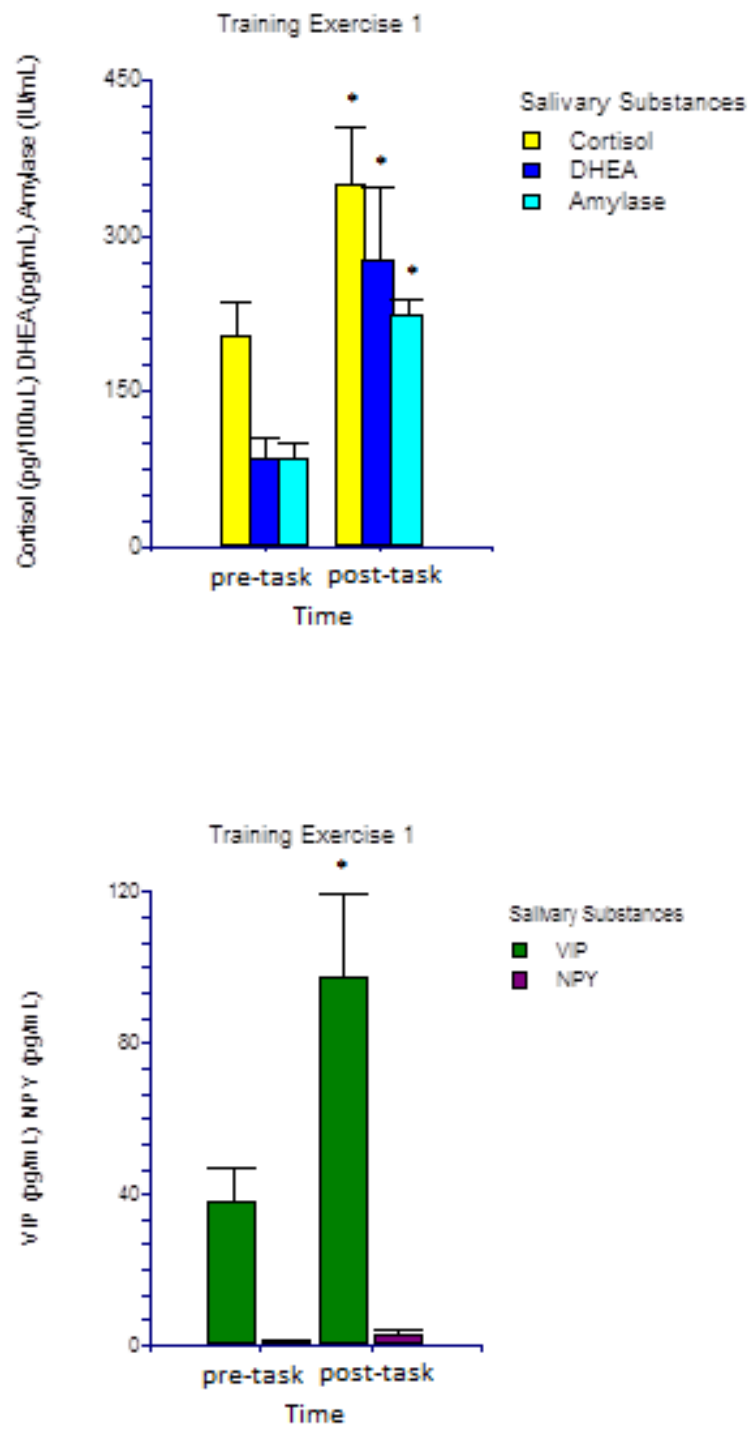

B

Fig. 10 (A) Training exercise 1- s-cortisol, s-DHEA and s-amylase after the obstacle course as compared to before the obstacle course $(n=9)$. (B) s-VIP and s-NPY after the obstacle course as compared to before the obstacle course $(n=9)$. The figure was adopted from Ventre et al. (2013). 
Table 15. Results: Training exercise 1: Linear regressions

\begin{tabular}{|l|l|}
\hline Correlation & Linear regression \\
\hline cortisol vs. amylase $(+)$ & $\mathrm{R}^{2}=0.4355, \mathrm{~F}(1,17)=12.34, \mathrm{p}=0.0029$ \\
\hline DHEA vs. $\mathrm{d} / \mathrm{c}(+)$ & $\mathrm{R}^{2}=0.6360, \mathrm{~F}(1,17)=27.95, \mathrm{p}<0.0001$ \\
\hline DHEA vs. NPY $(+)$ & $\mathrm{R}^{2}=0.4237, \mathrm{~F}(1,17)=11.76, \mathrm{p}=0.0034$ \\
\hline VIP vs. amylase $(+)$ & $\mathrm{R}^{2}=0.4411, \mathrm{~F}(1,17)=12.62, \mathrm{p}=0.0026$ \\
\hline VIP vs. DHEA $(+)$ & $\mathrm{R}^{2}=0.3394, \mathrm{~F}(1,17)=8.21, \mathrm{p}=0.0112$ \\
\hline VIP vs. cortisol $(+)$ & $\mathrm{R}^{2}=0.4190, \mathrm{~F}(1,17)=11.53, \mathrm{p}=0.0037$ \\
\hline cortisol vs. tension $(+)$ & $\mathrm{R}^{2}=0.4400, \mathrm{~F}(1,17)=12.57, \mathrm{p}=0.0027$ \\
\hline cortisol vs. energy $(+)$ & $\mathrm{R}^{2}=0.2693, \mathrm{~F}(1,17)=5.89, \mathrm{p}=0.027$ \\
\hline DHEA vs. tiredness $(-)$ & $\mathrm{R}^{2}=0.2315, \mathrm{~F}(1,17)=4.82, \mathrm{p}=0.0432$ \\
\hline DHEA vs. amylase $(+)$ & $\mathrm{R}^{2}=0.3123, \mathrm{~F}(1,17)=7.26, \mathrm{p}=0.0159$ \\
\hline amylase vs. tense arousal $(+)$ & $\mathrm{R}^{2}=0.2740, \mathrm{~F}(1,17)=6.04, \mathrm{p}=0.0258$ \\
\hline amylase vs. energetic arousal $(+)$ & $\mathrm{R}^{2}=0.3568, \mathrm{~F}(1,17)=8.87, \mathrm{p}=0.0088$ \\
\hline amylase vs. tiredness $(-)$ & $\mathrm{R}^{2}=0.2469, \mathrm{~F}(1,17)=5.24, \mathrm{p}=0.0359$ \\
\hline amylase vs. energy $(+)$ & $\mathrm{R}^{2}=0.2933, \mathrm{~F}(1,17)=6.64, \mathrm{p}=0.0203$ \\
\hline VIP vs. energetic arousal $(+)$ & $\mathrm{R}^{2}=0.2606, \mathrm{~F}(1,17)=5.64, \mathrm{p}=0.0304$ \\
\hline VIP vs. tension $(+)$ & $\mathrm{R}^{2}=0.2296, \mathrm{~F}(1,17)=4.76, \mathrm{p}=0.0442$ \\
\hline VIP vs. energy $(+)$ & $\mathrm{R}^{2}=0.3636, \mathrm{~F}(1,17)=9.14, \mathrm{p}=0.0081$ \\
\hline NPY vs. tense arousal $(+)$ & $\mathrm{R}^{2}=0.2561, \mathrm{~F}(1,17)=5.51, \mathrm{p}=0.0321$ \\
\hline
\end{tabular}

Training Exercise 2 
The moderate 1 hour exercise did not cause any significant changes in the salivary parameters measured. The statistics used was the Students two tailed paired T-Test. Lower cortisol levels after the exercise was probably due to its circadian rhythm (Fig. 11a). Salivary VIP levels were unchanged (Figure 11b). The Borg scale was significantly higher after the exercise $(\mathrm{p}<0.001)$ (Table 18). Significant Linear regressions are located in Table 16. 


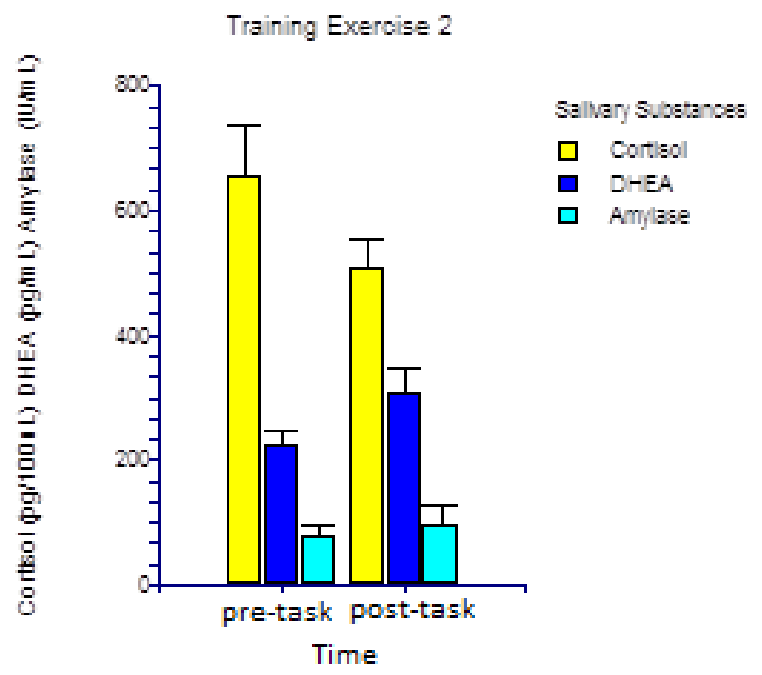

A

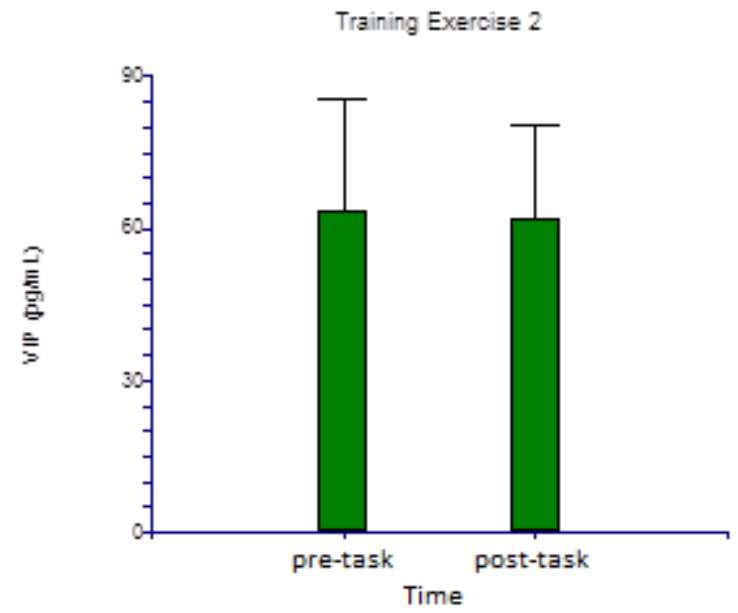

B

Fig. 11 (A) Training exercise 2-s-cortisol, s-DHEA and s-amylase after the moderate 1 hour exercise as compared to before the exercise $(n=6)$. (B) s-VIP after the moderate 1 hour exercise compared to before the exercise $(n=6)$. The figure was adopted from Ventre et al., (2013). 
Table 16. Results: Training exercise 2: Linear regressions

\begin{tabular}{|l|l|}
\hline Correlation & Linear regression \\
\hline DHEA vs. d/c $(+)$ & $\mathrm{R}^{2}=0.7665, \mathrm{~F}(1,11)=32.82, \mathrm{p}=0.0002$ \\
\hline DHEA vs. energetic arousal $(+)$ & $\mathrm{R}^{2}=0.3968, \mathrm{~F}(1,11)=6.57, \mathrm{p}=0.0281$ \\
\hline DHEA vs. tiredness $(-)$ & $\mathrm{R}^{2}=0.4135, \mathrm{~F}(1,11)=7.05, \mathrm{p}=0.0241$ \\
\hline DHEA vs. energy $(+)$ & $\mathrm{R}^{2}=0.3350, \mathrm{~F}(1,11)=5.04, \mathrm{p}=0.0486$ \\
\hline
\end{tabular}




\section{Training Exercise 3}

The statistics used was the Students two tailed paired T-Test. The 12 mile hike with a 30 lb backpack produced a significant increase in salivary cortisol $(\mathrm{p}=0.033)$ levels only (Figure 12a). The other salivary parameters were either the same or as in the case of DHEA insignificantly higher. Salivary VIP levels were unchanged. (Figure 12b). The Borg index was increased significantly $(\mathrm{p}=0.00012)$ (Table 18). Significant Linear regressions are located in Table 17. 


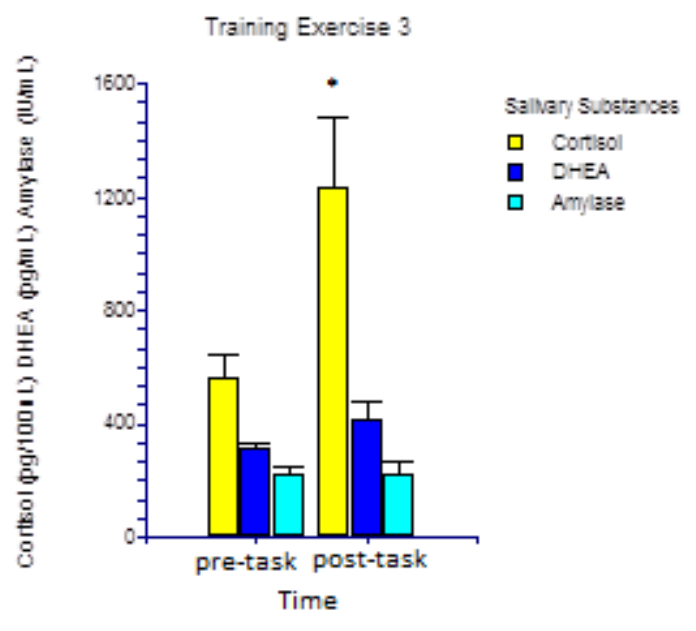

A

Training Exercise 3

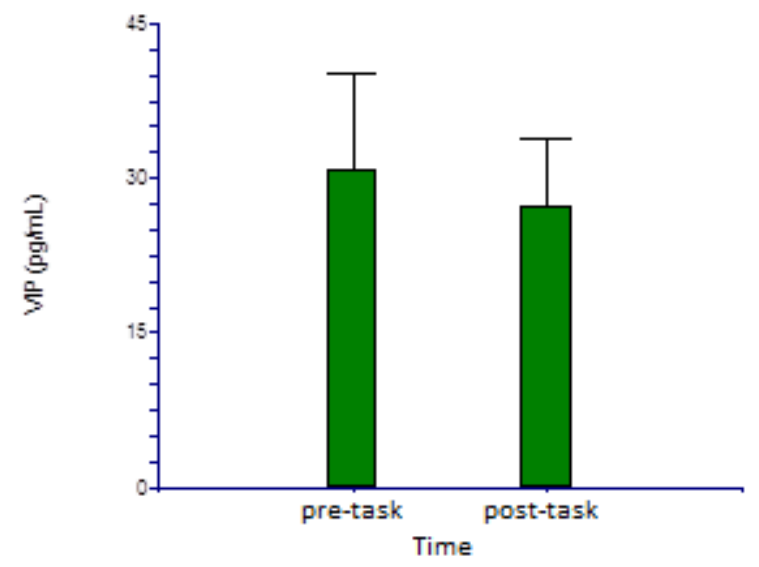

B

Fig. 12 (A)- Training Exercise 3- s-cortisol, s-DHEA and s-amylase after the 12 mile hike exercise as compared to before the exercise $(n=7)$. (B)- s-VIP after the 12 mile hike exercise as compared to before the exercise $(n=7)$. The figure was adopted from Ventre et al., (2013). 
Table 17. Results: Training exercise 3: Linear regressions

\begin{tabular}{|l|l|}
\hline Correlation & Linear regression \\
\hline cortisol vs. d/c (-) & $\mathrm{R}^{2}=0.5908, \mathrm{~F}(1,13)=17.32, \mathrm{p}=0.0013$ \\
\hline calmness vs. DHEA (-) & $\mathrm{R}^{2}=0.3133, \mathrm{~F}(1,13)=5.47, \mathrm{p}=0.0374$ \\
\hline cortisol and Borg $(+)$ & $\mathrm{R}^{2}=0.4662, \mathrm{~F}(1,13)=10.48, \mathrm{p}=0.0071$ \\
\hline
\end{tabular}


Table 18. Borg results for Training Exercises $1,2, \& 3$

\begin{tabular}{|l|c|c|}
\cline { 2 - 3 } \multicolumn{1}{c|}{} & \multicolumn{2}{c|}{ Borg } \\
\cline { 2 - 3 } \multicolumn{1}{c|}{} & before & $\underline{\text { after }}$ \\
\hline Exercise 1 & $12.0+/-0.91$ & $14.67+/-0.71 * *$ \\
\hline Exercise 2 & $6.83+/-0.48$ & $14.67+/-0.84 * * *$ \\
\hline Exercise 3 & $7.57+/-0.90$ & $17.14+/-0.91 * * *$ \\
& & \\
\hline
\end{tabular}




\section{Circadian results}

Repeated measures ANOVA revealed a significant peak at $0800 \mathrm{~h}$ in salivary cortisol $[(\mathrm{F} 5,47)=2.54, \mathrm{p}=0.0459]$ levels (Fig.13a). Tukey-Kraemer Multiple Comparison Test produced a significant difference between $0800 \mathrm{~h}$ and $2000 \mathrm{~h}$. There were no significant peaks detected in salivary DHEA $[\mathrm{F5}, 47)=1.73, \mathrm{p}=0.1530]$ levels (Fig. 13b). Salivary amylase $[\mathrm{F} 5,47)=2.43, \mathrm{p}=0.0544]$ levels demonstrated elevated but insignificant afternoon levels (Fig. 13c). Repeated measures ANOVA revealed a significant peak at 0800h in salivary VIP [(F5,47)=8.20, p=0.000033] levels (Fig. 13d) Tukey-Kraemer Multiple Comparison Test produced a significant difference between 0800h and 1200h, 1600h, 2000h and 2400h . 

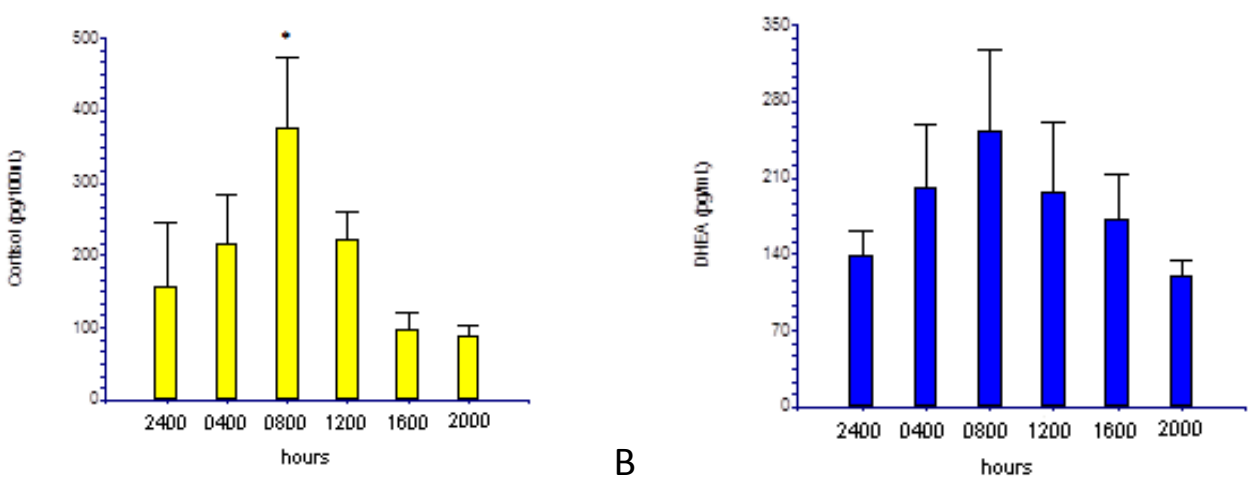

A

B
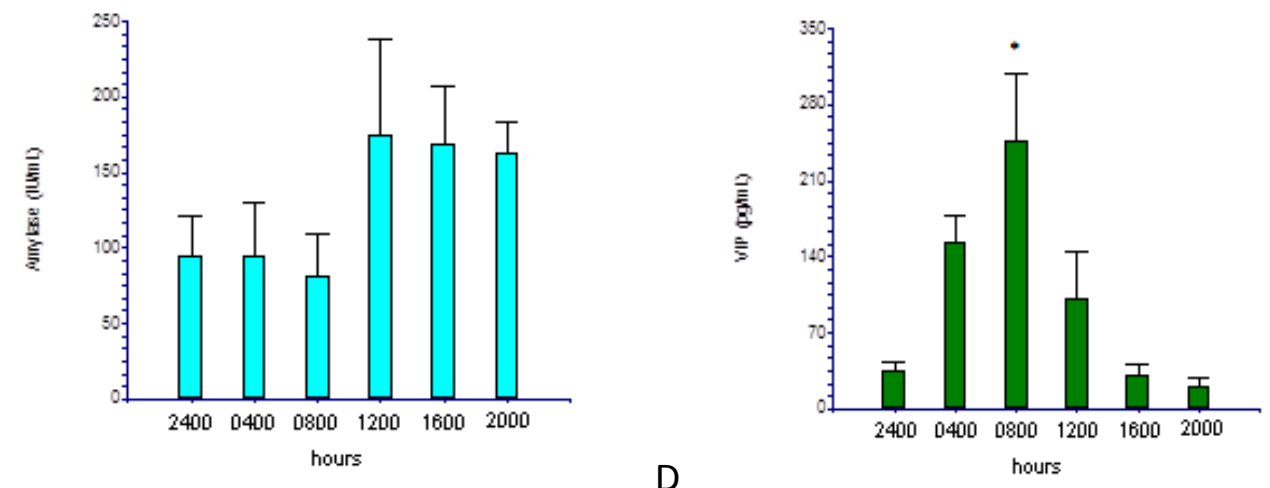

Fig. 13 Circadian studies: Levels of s-cortisol (A), s-DHEA (B), s-amylase (C) and s-VIP (D) taken at the following time points: $2400 \mathrm{hr}$., $0400 \mathrm{hr}$., $0800 \mathrm{hr} ., 1600 \mathrm{hr}$, and $2000 \mathrm{hr}$. Values are depicted as mean $+/$ - standard error $(n=8)$. Statistical significance was determined by repeated measures ANOVA followed by Tukey-Kraemer's multiple comparison test. 
Effect of drinking a bottle of water

Repeated measures ANOVA revealed a significant decrease in salivary cortisol $[\mathrm{F} 3,43)=8.08, \mathrm{p}=0.00043]$ levels at 10,20 and 30 minutes after drinking a bottle of water (Fig. 14a). Repeated measures ANOVA revealed a significant decrease in salivary DHEA $[\mathrm{F} 3,43)=3.60, \mathrm{p}=0.025]$ levels at 10 minutes after drinking a bottle of water (Fig. $14 \mathrm{~b})$. There were no significant changes in salivary amylase $[\mathrm{F} 3,43)=1.26, \mathrm{p}=0.305]$ (Fig. 14c) or salivary VIP $[\mathrm{F} 3,43)=1.75, \mathrm{p}=0.178]$ levels after drinking a bottle of water (Fig. 14d). 

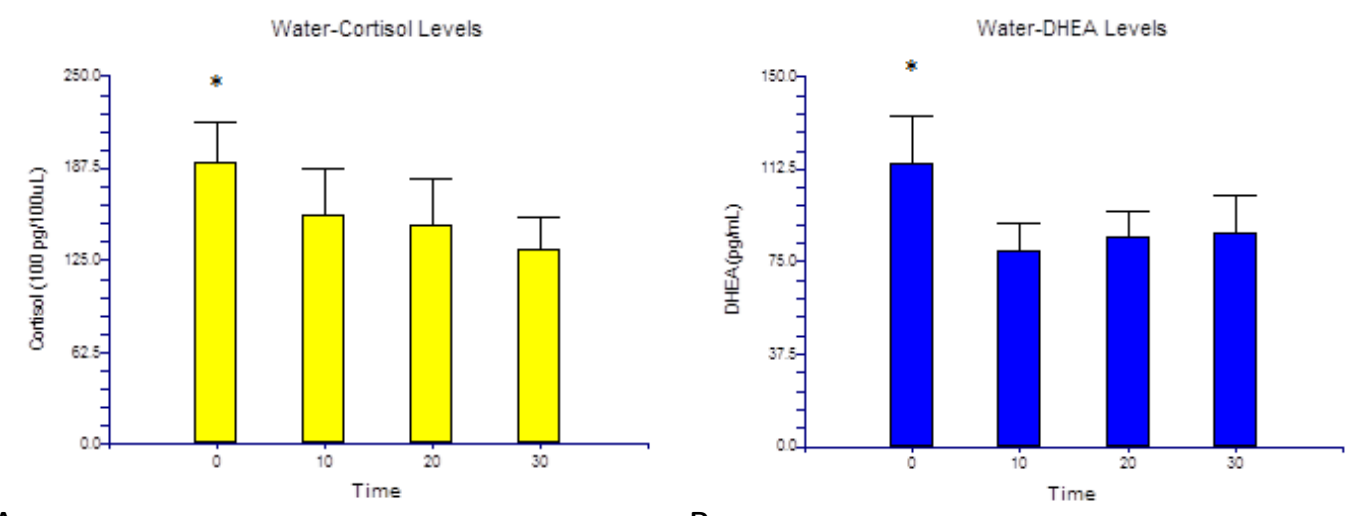

A

B
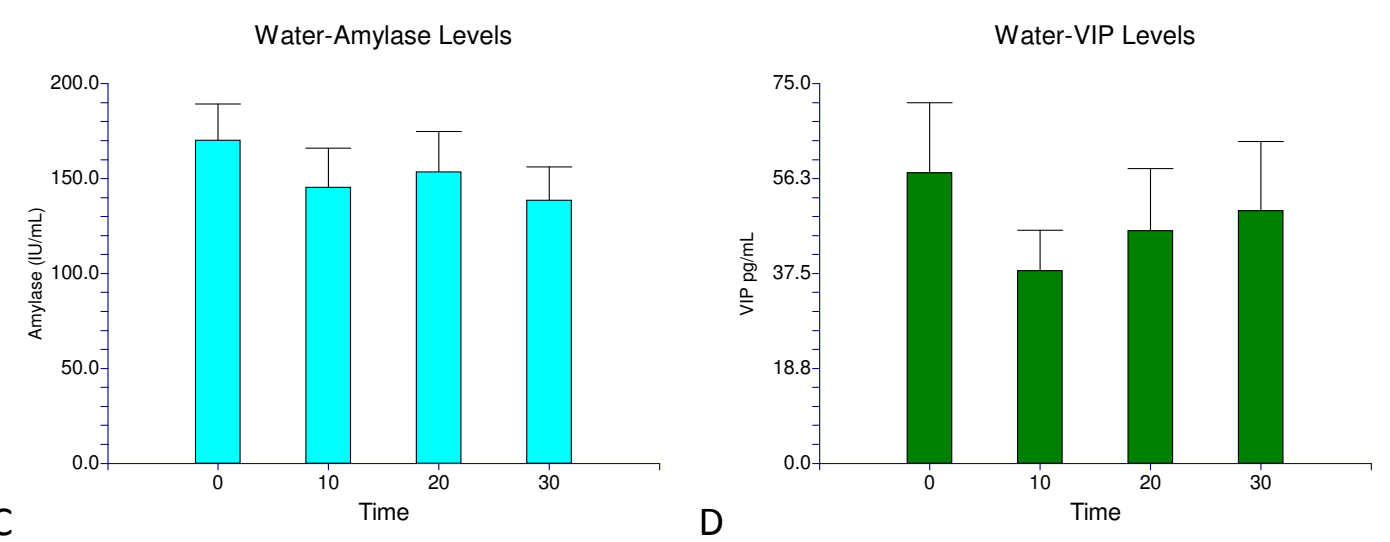

Fig. 14 Water Studies: Levels of s-cortisol (A), s-DHEA (B), s-amylase (C) and s-VIP (D) taken to study effect following the consumption of $20 \mathrm{oz}$. of Aqua-Fina water. Levels were assayed before water consumption ( 0 minutes) and 10, 20 and 30 minutes post water consumption. Values are depicted as mean $+/$ - standard error $(n=11)$. Statistical significance was determined by repeated measures ANOVA followed by Tukey-Kraemer's multiple comparison test. 
Effect of drinking a cup of coffee

There were no significant changes in salivary cortisol $[\mathrm{F} 3,43)=1.04, \mathrm{p}=0.389]$ (Fig. 15a), salivary DHEA $[\mathrm{F} 3,43)=0.62, \mathrm{p}=0.608]$ (Fig. 15b), salivary amylase $[\mathrm{F} 3,43)=0.20, \mathrm{p}=0.897]($ Fig. 15c) or salivary VIP $[\mathrm{F} 3,43)=1.31, \mathrm{p}=0.289]($ Fig. 15d) levels after drinking a cup of coffee. 

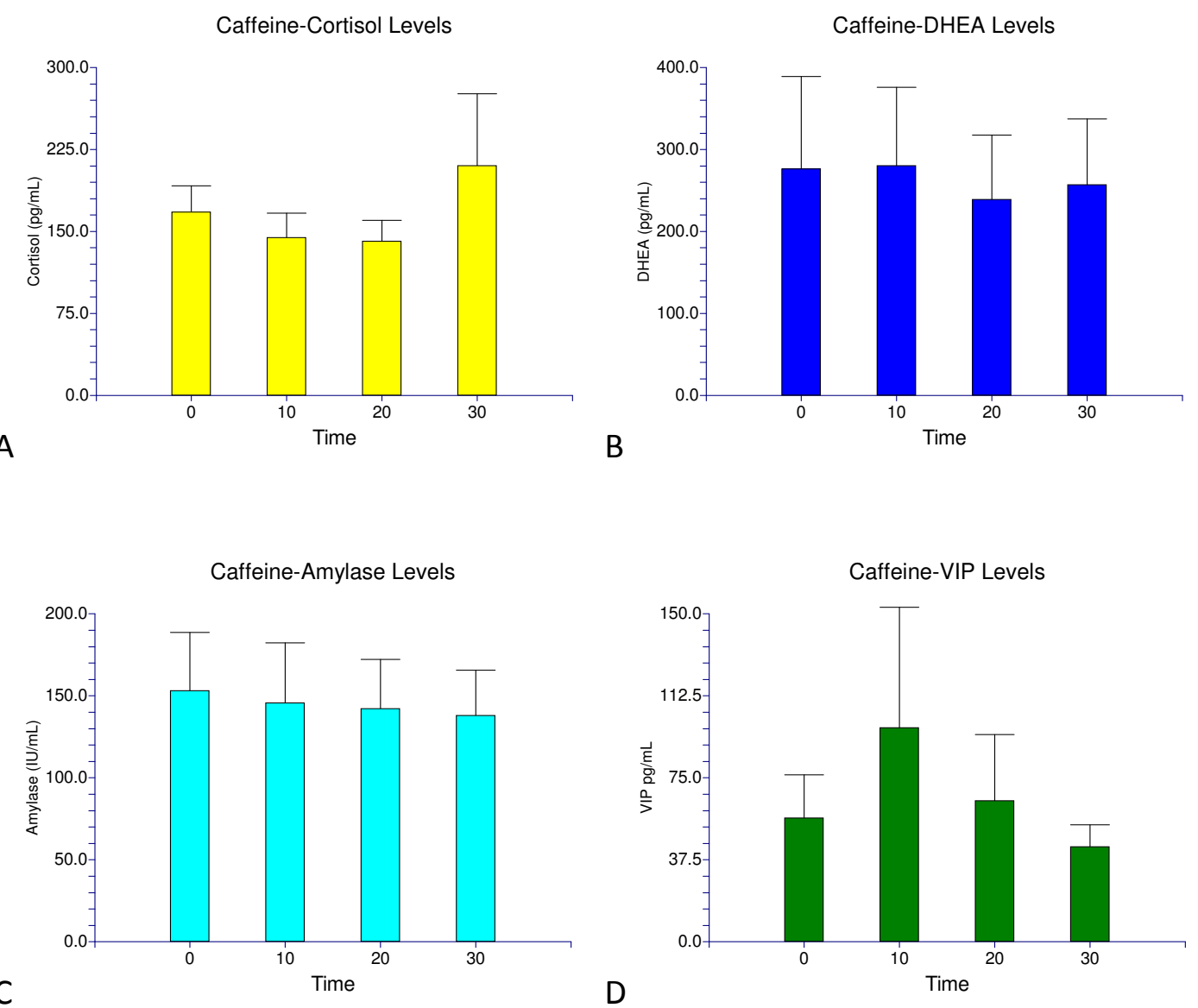

Fig. 15 Levels of s-cortisol (A), s-DHEA (B), s-amylase (C) and s-VIP (D) taken to study effect following the consumption of a regular medium coffee (starbucks breakfast blend). Levels were assayed before coffee consumption ( 0 minutes) and 10, 20 and 30 minutes post water consumption. Values are depicted as mean $+/$ - standard error $(n=11)$.

Statistical significance was determined by repeated measures ANOVA followed by Tukey-Kraemer's multiple comparison test. 


\section{Discussion}

In order to perform these studies, it was first determined that NPY and VIP could be detected in saliva and that we could obtain reasonable recovery from addition of peptide to saliva. One hundred $\mathrm{mM}$ Na Citrate proved to better than the other additives: EDTA, trasylol, $\mathrm{HCl}$, TFA, or even $10 \mathrm{mM}$ NaCitrate. Subsequent saliva studies spiked with cold NPY or VIP yielded approximately $90 \%$ recovery as determined by RIA. Based on this data we decided to use $100 \mathrm{mM}$ NaCitrate as the preservative for the stress studies. The levels detected in saliva for NPY and VIP were not consistent with the relative levels in plasma. In saliva there was considerably more VIP than NPY which is the opposite of plasma. The probable explanation is due to higher levels of VIP expression in the parasympathetic nerves. S-NPY expression is restricted to the certain blood vessels which feed the sympathetic nerves (Kontinnen et al., 1992). We focused on VIP in these studies because we could detect it and possibly observe elevations.

The stress system which involves the HPA axis and the ANS responds to stressors of any kind (Tsigos \& Chrousos, 2002). In the following studies, we evaluated salivary markers of both the neuroendocrine and the autonomic branches of the stress response system (Morgan et al., 2004; Nater et al.,2006; Chatterton et al.,1996; Santavirta et al.,1997). The main purpose of the studies was to determine if salivary VIP and/or salivary NPY responded to different types of stress. We mainly used psychological stressors using the common exam situation. However, we also used other situations which involved psychological stress such as the disaster drill (PAD) and graduate 
presentations: seminar or thesis defense. Physical stressors were involved in 3 different studies using ROTC cadets: Training Exercises 1,2 and 3. All of these ROTC studies had a significant physical stress component and this was observed in the Borg scale results, however we believe that Training exercise 1; a situation where the students were actually competing and trying to impress their commanding officers may have involved a significant psychological component. Training exercise 1 which was the shortest in duration involved both psychological and physical stress as an obstacle course was involved. In addition, we observed a significant regression between cortisol and tension in training exercise 1 (Table 15). Also, VIP was correlated positively with tension in exercise 1 (Table 15). Training exercise 2 involved moderate physical exercise which lasted about 1 hour. Training exercise 3 involved mostly physical stress which lasted approximately 3 hours which could be categorized as extreme physical exertion. There also could have been a psychological component; however, training exercise 3 produced a significant regression between cortisol and the Borg scale.

When evaluating the data from the different studies it is important to focus on whether the stressor was strong enough to elicit an HPA and/or autonomic response. In order to do this, we determined the salivary levels of cortisol (HPA), DHEA (HPA) and amylase (sympathetic branch of ANS). Since the neuropeptide data has not been properly validated as of yet, it is important to ask the question is the neuropeptide data physiologically relevant? Thus we correlated the neuropeptide data with established stress markers salivary cortisol, DHEA and amylase. 


\section{PAD study}

The volunteers for the disaster drill were the people who organized and were in charge of the event itself; they were not the people who took part in the various disaster scenarios. Salivary parameters showed no increase after vs. before the disaster drill. In fact, salivary cortisol was significantly less due to salivary cortisol's circadian rhythm. DHEA levels were also significantly less post drill. Thus, there was no stress response in these subjects and actually we noted the standard circadian decrease from early morning to afternoon.

\section{Exam Stress}

Exam stress was used as a pure psychological stress with no physical component.

For E1, salivary cortisol levels on both mornings of approximately (900pg/100ul) are not normal levels and are actually in the stress range. Therefore, we cannot use this data.

For E2, there were no significant elevations in s-cortisol, s-DHEA, s-amylase, sNPY and s-VIP when comparing exam data with control data. Thus, there was no stress response to the exam.

For E3, there were no significant changes in s-cortisol, s-DHEA and s-amylase. Thus, there was no stress response to the exam. There was a significant elevation in postcontrol s-VIP vs. pre-control s-VIP. All the levels seemed low and it was uncertain as to whether or not this data is meaningful. S-NPY post-control, was higher than post-exam. 
There are no noteworthy changes for E4. There were no significant changes for any of the salivary parameters with respect to exam data vs. control data. Cortisol precontrol was significantly higher than after control but it is known to be elevated for that time of day.

For E5, there were no noteworthy significant changes for cortisol and DHEA. Amylase showed a significant elevation when comparing pre-exam with pre-control samples.

In conclusion, we do not see anticipatory stress or a stress response elicited by taking an exam in any of our exam paradigms. We did note increased tension in E2, E3 and E5.

\section{Training exercises}

The current studies suggest that salivary VIP responds to stress. The VIP response in training exercise 1 paralleled the response by salivary cortisol, salivary amylase and salivary DHEA. In fact, significant regressions were obtained between VIP and the other 3 salivary markers. The mild exercise routine, training exercise 2 , did not elicit a significant increase in any of the parameters even though the borg index was significantly increased. Training exercise 3 , the 3 hour back-pack hike, resulted in a significant increase in salivary cortisol only. The borg index was increased in all 3 training exercises which suggests that physical exertion was a factor in all 3 training exercises. Salivary NPY was also investigated as a marker for stress, however the results 
suggest that salivary NPY levels are very low and may not be suitable as a salivary marker for stress.

We investigated salivary VIP as a possible salivary marker for parasympathetic activity based on published results which identified parasympathetic nerves containing VIP which enervate salivary glands. The autonomic system is known to respond to stress. The two divisions of the ANS are dominant under different conditions (McCory, 2007). The SNS is active during the classic evolutionary "fight or flight" reactions and during exercise in order to meet the cardiovascular and energy demands of the body (McCory, 2007; Hautala et al., 2009). The PNS is active in times of rest but also restores homoeostasis once the stressor has subsided. (Porges et al., 2009, 1995; Sahar et al., 2001). This restorative function is best exemplified by the vagal motor pathways on the cardiac pacemaker which slows the heart, inhibits fight-flight mechanisms on the SNS, reduces HPA axis activity (cortisol), and reduces inflammation (Porges et al., 2009, 1995, Sahar et al., 2001). Most research involving VIP and physical/psychological stress measured plasma VIP only (Oktedalen et al., 1983-1; Oktedalen et al., 1983-2; Crozier, et al., 1988; Opstad et al., 1987; Galbo et al., 1979). Prior research suggests that the VIP increase in the plasma was a hormonal response to low blood glucose levels (Oktedalen et al., 1983-1; Oktedalen et al., 1983-2; Crozier, et al., 1988; Opstad et al., 1987; Galbo et al., 1979). For example, Oktedalen et al. measured plasma VIP in well trained men after a $90 \mathrm{~km}$ cross country ski race and found plasma VIP elevated after the race and the levels returned to normal after 140 min after rest (Oktedalen et al., 1983-1). However, in the same study participants who were given a glucose load after the race saw their VIP levels 
return to normal after 60 minutes. In fact, Oktedalen et al. (1983-1) has suggested from this study and others that VIP may have a role as "a polypeptide of substrate need"(Oktedalen et al., 1983-1, Oktedalen et al., 1983-2). VIP may be important in the mobilization of energy stores in times of need. In this study, salivary VIP was increased significantly after the most challenging training exercise 1 which was the obstacle course race. The fact that the obstacle course caused a significant increase in all the salivary parameters is very suggestive that it was the most stressful. However, here the race lasted only 10-15 minutes, and can be described as acute stress and hardly the 4-6 hours it took for Oktedalen's 90km cross country ski race. Increased salivary levels of VIP in our study may indicate increased parasympathetic activity or tone in order to restore homeostasis.

The results from the 3 separate studies suggested that they were of different intensity and as such could have elicited different responses. However, there are caveats to consider such as timing since initiation of stress and time of day. The timing of saliva collection after the initiation of stress was not the same for the 3 different studies because the duration of stress was different. The literature suggests that salivary cortisol peaks around 20 minutes and salivary amylase peaks around 10 minutes after the initiation of stress (Gaab et al., 2003; Kirschbaum et al., 1999; Kudielka et al., 2004; Nater et al., 2005; Schommer, Hellhammer and Kirschbaum 2003; Takai et al., 2004). For example, this could affect our interpretation of the post salivary cortisol levels of training exercise 1 which was collected 10 minutes after the initiation of stress and presumably could have been higher at 20 minutes. The relative contribution of the nature and the degree of the 
stresses for the 3 training exercises should also be considered i.e. psychological vs. physical. Another limitation of the study relates to circadian variations as training exercise 1 took place at 1700 whereas training exercise 2 and 3 took place in the morning (0700 \& 0600 respectively). Circadian rhythms have been described for cortisol, DHEA and amylase: cortisol levels are highest in the morning and decrease progressively for the rest of the day (Morgan et al., 2004; Brown et al., 2008; Hucklebridge et al., 2005); DHEA levels also peak in the morning followed by a less pronounced decrease than cortisol (Hucklebridge et al.; 2005, Netherton et al., 2004) and finally research suggests amylase is lowest in the morning and higher in the afternoon and evening (Nater et al., 2007; Nater et al., 2009). Therefore, comparing our baseline results with the literature, we find good agreement for salivary cortisol and salivary DHEA from the different training exercises with the literature. We did not observe the diurnal variation for salivary amylase that Nater et al. observed with levels being higher in the afternoon than in the morning, but our range of baseline levels are in the range of literature values (Nater et al., 2005; Nater et al., 2007; Rohleder et al., 2004; van Stegeren et al., 2006). It is possible that the higher salivary amylase for exercise 3 reflects anticipatory stress as well as post stress. Taking the circadian effects into account it is a possibility that higher baseline levels of cortisol and DHEA may blunt or mask the stress response, and as such, could confound our results. A consideration would be did training exercise 2 and 3 overlap significantly with the cortisol awakening response (Kudielka et al., 2009). In addition higher baseline morning DHEA levels could confound the results, as Morgan et al. has suggested, higher DHEA levels are associated with better performance by military 
subjects and Izawa et al. has suggested that higher DHEA levels are associated with better mood (Morgan et al., 2004; Morgan et al., 2009; Izawa et al., 2008). Indeed, it is these findings which encouraged and reinforced the reason to study salivary DHEA with salivary cortisol in this study as DHEA has been described as anxiolytic with physiological effects antagonistic to that of cortisol. The question then remains if all 3 training exercises were initiated in the afternoon or evening would the salivary results been different.

Another noteworthy caveat is that we are measuring an isolated or paracrine source of VIP and NPY as opposed to measuring these peptides in plasma, and therefore the physiological significance can be questioned. Therefore, we attempted to make correlations with the established salivary stress markers where possible. The results from this study suggest that baseline salivary VIP-IR levels are at least 10 fold higher than baseline salivary NPY-IR levels. This is not in agreement with the plasma levels of VIP and NPY. Oktedalen et al. reports plasma VIP levels of 30-100pg/mL whereas Morgan et al. reports plasma NPY levels of 50-200 pg/mL (Morgan et al., 2000; Oktedalen et al., 1983). The amount of salivary NPY relative to salivary VIP observed in our study is much less than that observed in plasma (Morgan et al., 2000; Oktedalen et al., 1983). In the periphery NPY is co-localized in peripheral sympathetic neurons and is also contained in and possibly released from the adrenal medulla. Peripheral sources of VIP are parasympathetic nerves, gut and pancreas. We also performed NPY and VIP percent recovery studies, and $90 \%$ recovery was obtained for both peptides from saliva, therefore the low levels of salivary NPY observed was not due to degradation. So why the 
discrepancy between salivary levels and plasma levels of the two peptides? A possibility is the relative distribution of the peptides in salivary glands. For example, Konttinen found in labial salivary glands the Acini, intralobular ducts, small arteries and post capillary veins were richly innervated by VIP-IR fibers whereas NPY-IR was restricted to blood vessels (Konttinen et al., 1992). This difference in distribution could account for the difference in salivary VIP-IR and salivary NPY-IR that we have observed in this study. In addition the values of salivary VIP-IR and salivary NPY-IR obtained in this study are comparable to those obtained by Santavirta et al. (Santavirta et al., 1997). VIP is known to be released from parasympathetic nerves and previous research involving plasma VIP and stress suggests it responds to extreme psychological/physical stress: prolonged fasting, withholding sleep etc (Oktedalen et al., 1983-1; Oktedalen et al., 1983-2; Crozier, et al., 1988; Opstad et al., 1987; Galbo et al., 1979). VIP levels in saliva have been detected by others, and in one study with Sjogren's syndrome and normal healthy subjects, stress was associated with higher salivary VIP concentrations (Santavirta et al., 1997). In training exercise 1, VIP levels did significantly correlate with stress biomarkers cortisol, amylase and DHEA. Some of the functions of the parasympathetic nervous system is to counter that of the sympathetic system. Increased VIP release from the salivary parasympathetic nerves in response to stress may be an indicator that the body is experiencing a certain threshold and/or specific nature of stress e.g. psychological vs. physical.

The development of new biomarkers for stress is needed even though cortisol, amylase and DHEA are the most widely used parameters. Salivary markers cortisol and 
DHEA indicate HPA axis activation while salivary amylase correlates with sympathetic activation. "Since stress can be multifaceted it requires a multidimensional measurement approach" (Nater et al., 2009). Markers which help distinguish between psychological and physical stress are needed as well as markers indicating the severity of the stress experienced. Our results may not establish VIP as a putative salivary stress biomarker however it merits further research.

Salivary NPY levels are very low and most of the time undetectable. Training exercise 1 study was the only ROTC study that NPY levels were measured. Even though they were insignificantly elevated after the obstacle course, positive correlations were realized with amylase and VIP. Salivary amylase is believed to be a marker for sympathetic nervous system activity. Our working hypothesis is that salivary NPY is released from the sympathetic nerves adjacent to the salivary glands. Correlations between NPY and VIP were also observed in the PAD study and two exam studies (E1 \& E2).

The results for VIP and NPY as responding to stress is somewhat encouraging, more so for VIP than NPY because VIP levels are higher than NPY and thus easier to detect. Salivary NPY levels are too low to be assayed with reliability. Therefore, salivary NPY is not suitable as a stress indicator. The fact that elevated VIP levels were observed in individuals exposed to a psychological stressor such as public speaking or exam is encouraging. Elevated VIP levels were not observed in individuals who were exposed predominately to physical stressors such as training exercise 2 and 3 . Training 
exercise 1 is believed to have involved both psychological and physical stress. This study had significant elevations in 4 out of 5 markers: cortisol, DHEA, Amylase \& VIP.

\section{Circadian studies}

In the literature, circadian rhythms have been reported for salivary cortisol and salivary DHEA (Hucklebridge et al., 2005). Cortisol levels are highest in the morning and decrease progressively for the rest of the day (Morgan et al., 2004; Brown et al., 2008; Hucklebridge et al., 2005). DHEA levels also peak in the morning followed by a less pronounced decrease than cortisol (Hucklebridge et al., 2005; Netherton et al., 2004). Also, according to the literature, salivary amylase is lowest in the morning and highest in the afternoon and evening (Nater et al., 2007; Nater et al., 2009). Therefore, comparing our circadian results with the literature we find good agreement for salivary cortisol. Salivary cortisol levels demonstrated a circadian rhythm which revealed a significant peak at 0800h (Morgan et al., 2004; Brown et al., 2008; Hucklebridge et al., 2005). Salivary DHEA levels demonstrated a circadian rhythm with an insignificant peak at 0800h (Hucklebridge et al., 2005; Netherton et al., 2004). Salivary amylase levels were lower in the morning than in the afternoon which is consistent with the literature (Nater et al., 2007; Nater et al., 2009). Salivary VIP levels demonstrated a circadian rhythm with a significant peak at $0800 \mathrm{~h}$. There are no studies looking at the circadian rhythm of salivary VIP levels. However, Lockinger et al. report a plasma VIP circadian with levels that peak at 3:00am (Lockinger et al., 2004). 
Effect of drinking a glass of water

Salivary cortisol levels were decreased after consumption of water. Significant decreases at 10,20, and 30 minutes when compared to 0 minutes. Salivary DHEA levels were also decreased after water consumption at 10 minutes. Salivary amylase levels were insignificantly reduced after water consumption. Likewise, salivary VIP also demonstrated an insignificant decrease post water consumption. The literature is scarce on the effect of water on salivary parameters. Water has been shown to stimulate saliva flow rate albeit to a lesser extent than chewing gum and drinking fizzy cola or black tea. (Davies et al., 2009). Therefore, increased flow rate could conceivably affect the levels of certain salivary substances. A review on salivary stress markers by Konen Obayashi states that saliva flow rate has no impact on salivary cortisol and salivary amylase levels (Obayashi, 2013). Rohleder at al. also reports that stress-induced increase in salivary alpha-amylase is independent of saliva flow rate (Obayashi, 2013; Rohleder et al., 2006).

\section{Effect of drinking a cup of coffee}

"Caffeine acts as an important modulator of cardiovascular and central nervous system activity" (Lovallo et al., 2006). Our results of drinking a cup of coffee on salivary cortisol are somewhat consistent with literature which reports no effect of caffeine on salivary cortisol levels in the absence of stress (Lovallo et al., 2006). There was no significant increase in salivary cortisol levels after drinking a cup of coffee. We also observed similar results in regard to salivary DHEA with no significant changes for all time points. Salivary amylase also did not display any significant changes post caffeine 
consumption. This is in disagreement with the Bishop et al. data (Bishop et al., 2006). However, the Bishop group saw an increase in amylase activity 1 hour post caffeine consumption. Our times were limited 30 minutes post caffeine consumption. There were no significant changes in salivary VIP levels.

\section{Conclusion}

A brief intense training exercise lasting minutes elicited a stress response with significant increases in salivary cortisol, DHEA, amylase and VIP. Linear regressions demonstrate significant correlations between VIP with cortisol, amylase and DHEA. A less rigorous exercise did not elicit a stress response with no significant increases in salivary cortisol, DHEA, amylase and VIP. A longer intense exercise lasting hours elicited a stress response with significant increases only in salivary cortisol. The long time course of this response may be responsible for not detecting the autonomic response. Although there was a significant increase in the Borg for all three exercises, tension from the Thayer activation deactivation checklist only correlated with cortisol in exercise 1.

The studies examining the effect of taking an exam did not yield significant increases in salivary cortisol, DHEA, amylase or VIP. It is possible that the student population consisted of high responders and low responders or anticipatory stress vs. post exam stress which might have masked an effect. In addition, it is possible that the time course waiting for conclusion of the exam is too long or that taking exams had become routine for the students. 
It is interesting to note that there is a circadian rhythm of salivary VIP levels that peaks with salivary cortisol but not with salivary amylase. Further studies need to be performed with regard to the effect of drinking water or coffee on salivary cortisol, DHEA, amylase and VIP with a longer time course. If salivary cortisol actually does drop after drinking water, the data presented here suggests that the lack of effect reported here after coffee drinking might actually be masking an increase in coffee drinking. 


\section{References}

Ahn, R.S., Lee, Y.J., Kwon, H.B., Chun, S. (2007) Salivary cortisol and DHEA levels in the Korean population: Age-related difference, diurnal rhythm, and correlations with serum levels. Yonsei Medical Journal, 48, 379-388.

Al-Ayadhi, L.Y. (2005) Neurohormonal changes in medical students during academic stress. Annals of Saudi Medicine. 25:1:36-40, 2005.

Allen, R., Boublik, J., Hauger R., Scott, N., Rivier, J., Brown, M. (1991)

Neuropeptide Radio-immunoassay:Characterization and Application. Clinical and Experimental Pharmacology and Physiology, 18, 825-833.

Albo, F., Antonangeli, R., Cavazza, A., Marini, M., Roda, L.G., Rossi, P. (2001) Neuropeptide degradation in naïve and steroid-treated allergic saliva. International Immunopharmacology, 1, 1777-1788.

Albo, F., Antonangeli, R., Cavazza, A., Marini, M., Roda, L.G., Rossi, P. (2002) Neuropeptide enzyme hydrolysis in allergic human saliva, Peptides, 23, 185-192.

Andrade, D., Assis, D.M., Santos, J.A.N., Alves, F.M., Hirata, I.Y., Araujo, M.S., Blaber, S.I., Blaber, M., Juliano, M.A., Juliano, L. (2011) Substrate specificity of Kallikrein-related peptidase 13 activated by salts or glycosaminoglycans and a search for natural substrate candidates. Biochimie. 93, 1701-1709.

Bellamy, J.L., Cady, R.K., Durham, P.L. (2006) Salivary levels CGRP and VIP in rhinosinustis and migraine patients, Headache, 46, 24-33.

Birkett, M.A. The trier social stress protocol for inducing psychological stress. (2011) The Journal of Visual Experimentation, 56, 3238.

Bishop, N.C., Walker, G.J., Scanlon, G.A., Richards, S., Rogers, E. (2006) Salivary IgA responses to prolonged intensive exercise following caffeine ingestion. Medicine and Science in Sports and Exercise, 38(3), 513-519, 2006.

Borg, G. (1998) Borg's perceived exertion and pain scales. Human Kinetics, Champaign, IL.

Brown, G.L., McGarvey, E.L. Shirtcliff, E.A., Keller, A., Granger, D.A., Flavin, K. (2008) Salivary cortisol, dehydroepiandrosterone, and testosterone interrelationships in healthy young males: A pilot study with implications for studies of aggressive behavior. Psychiatry Research, 159, 67-76. 
Carrasco, G., Van de Kar. L. (2003) Neuroendocrine pharmacology of stress. European Journal of Pharmacology, 463, 235-272.

Chatterton, R.T., Vogelsong, K.M., Lu, Y., Ellman, A.B., Hudgens, G.A. (1996)

Salivary alpha-amylase as a measure of endogenous adrenergic activity. Clinical Physiology, 16, 433-448.

Chrousos, G.P., Gold, P.W. (1992) The concept of stress and stress system disorders: Overview of physical and behavioral homeostasis, Journal of American Medical Association, 267(9), 1244-1252.

Crozier, T.A., Drobnik, L., Stafforst, D, Kettler, D. (1988) Opiate modulation of the stress induced increase of vasoactive intestinal peptide (VIP) in plasma. Hormone Metabolism Research, 20(6), 652-656.

Cugini, P., Lucia, P., DiPalma, L., Re, M., Canova, R., Gasbarrone, L., Cianetti, A. (1992) The circadian rhythm of atrial natriuretic peptide, vasoactive intestinal peptide, beta-endorphin and cortisol in healthy young and elderly subjects. Clinical Autonomic Research, 2, 113-118.

Culp, D.J., Richardson, L.A. (1996) Regulation of mucous acinar exocrine secretion with age. Journal of Dental Research, 75(1), 575-580.

Davies, G.A., Wantling, E., Stokes, J.R. (2009) The influence of beverages on the stimulation and viscoelasticity of saliva: Relationship to mouthfeel? Food Hydrocolloids, 23, 2261-

2269.

Dawidson, I., Blom, M., Lundeberg, T., Theodorsson, E., Angmar-Mansson, B. (1997) Neuropeptides in the saliva of healthy subjects. Life Sciences, 60, 269-278.

De Kloet, C.S. Vermetten, E., Geuze, E., Kavelaars, A., Heijnen, C. Westenberg, H. (2006) Assessment of HPA-axis function in posttraumatic stress disorder: Pharmacological and non-pharmacological challenge tests, a review. Journal of Psychiatric Research, 40, 550-567.

Feher, E., Zelles, T., Nagy, G. (1999) Immunocytochemical localization of neuropeptide-containing nerve fibres in human labial glands, Archives of Oral Biology, 44, S33-S37.

Galbo, H., Hilsted, J., Fahrenkrug, J., Schaffalitzky De Muckadell, O.B. (1979) Fasting and prolonged exercise increase vasoactive intestinal polypeptide (VIP) in plasma. Acta Physiologica Scandinavica, 105(3), 374-377. 
Gaab, J., Blattler, N., Menzi, T., Pabst, B., Stoyer, S., Ehlert, U. (2003) Randomized controlled evaluation of cognitive-behavioral stress management on cortisol responses to acute stress in healthy subjects. Psychoneuroendocrinology, 28, 767-779.

Hamer, M., Steptoe, A. (2007) Association between Physical Fitness, Parasympathetic Control and Proinflammatory Responses to Mental Stress. Psychosomatic Medicine, 69, 660-666.

Hammerfald, K., Grau, M., Kinsperger, A., Zimmermann, A., Ehlert, U., Gaab, J. (2006) Persistent effects of cognitive-behavioral stress management on cortisol responses to acute stress in healthy sunjects- A randomized controlled trial. Psychoneuroendocrinology, 31, 333-339.

Han, S., Chen, X., Yang, C.L., Vickery, L., Wu, Y., Nais, L., Macarthur, H., Westfall, T.C. (2005) Influence of cold stress on neuropeptide Y and sympathetic neurotransmission. Peptides, 26, 2603-2609.

Hautala, A.J. Kiviniemi, A.M., Tulpo, M.P. (2009) Individual responses to aerobic exercise: The role of the autonomic nervous system. Neuroscience and Biobehavioral Reviews, 33, 107-115.

Heilig, M. (2004) The NPY system in stress anxiety and depression. Neuropeptides, 38, 213-224.

Hellhammer, D.H., Wust, S., Kudielka, B.M. (2009) Salivary cortisol as a biomarker in stress research. Psychoneuroendocrinolgy, 34, 163-171.

Herman, J., Cullinan, W. (1997) Neurocircuitry of stress: central control of the hypothalamo-pituitary-adrenocortical axis. Trends in Neuroscience, 20, 78-84.

Hintze, J.L. (1998) Number cruncher statistical systems, Kaysville, Utah.

Hucklebridge, F., Hussain, T., Evans, P., Clow, A. (2005) The diurnal patterns of the adrenal steroids cortisol and dehydrepiandrosterone (DHEA) in relation to awakening. Psychoneuroendocrinology, 30, 51-57.

Hunter, L., Rorie, D., Yaksh, T., Tyce, G. (1998) Concurrent Separation of Catecholamines, Dihydroxyphenylglycol, Vasoactive Intestinal Peptide, and Neuropeptide Y in superfusate and tissue extract. Analytical Biochemistry, 173, 340-352.

Igarashi, H., Fujimori, N., Ito, T., Nakamura, T., Oono, T., Nakamura, K., Suzuki, K. (2011) Vasoactive intestinal peptide (VIP) and VIP receptors: Elucidation of structure and function for therapeutic applications. International Journal of Clinical Medicine, 2(4), 500-508. 
Izawa, S., Sugaya, N., Shirotsuki, K., Yamada, K.C., Ogawa, N., Ouchi, Y., Nagano, Y., Suzuki, K., Nomura, S. (2008) Salivary dehydroepiandrosterone secretion in response to acute psychosocial stress and its correlations with biological and psychological changes. Biological Psychology, 79, 294-298.

Jerjes, W., Cleare, A., Wessely, S., Wood, P., Taylor N. (2005) Diurnal patterns of salivary cortisol and cortisone output in chronic fatigue syndrome. Journal of Affective Disorders, 87, 299-304.

Jorde, R., Burhol, P.G. (1985) Diurnal profiles of gastrointestinal regulatory peptides. Scandinavian Journal of Gastroenterology, 20, 1-4.

Kirschbaum, C., Kudielka, B., Gaab, J., Schommer, N.C., Hellhammer, D.H. (1999) Impact of gender, menstrual cycle phase, and oral contraceptives on the activity of the hypothalamuspituitary-adrenal axis. Psychosomatic Medicine, 61, 154-162.

Konttinen, Y.T., Hukkanen, M., Kemppinen, P., Segerberg, M. Sorsa, T., Malmstrom, M., Rose, S., Itescu, S., Polak, J.M. (1992) Peptide-containing nerves in labial salivary glands in Sjogren's syndrome. Arthritis Rheumatology, 35(7), 815-820.

Konttinen, Y.T., Tornwall, J., Kemppinen, P., Uusitalo, H., Sorsa, T., Hukkanen, M., Polak, J.M. (1996) Neutral endopeptidase (EC3.4.24.11) in labial salivary glands in healthy controls and in patients with Sjogren's syndrome. Annals of the Rheumatic Diseases, 55, 513-519.

Kraemer, W., Ratamess, N. (2005) Hormonal responses and adaptations to resistance exercise and training. Sports Medicine, 35(4), 339-361.

Krieger, D.T., Allen, W., Rizzo, F., Krieger, H.D. (1971) Characterization of the normal temporal pattern of plasma corticosteroid levels. Journal of Clinical Endocrinology and metabolism, 32(2), 266-284.

Kudielka, B.M., Hellhammer, D.H., Wust, S. (2009) Why do we respond so differently? Reviewing determinants of human salivary cortisol responses to challenge. Psychoneuroendocrinology, 34, 2-18.

Kuo, L., Zukowska. Z. (2007) Stress, NPY and vascular remodeling: Implications for stress related diseases. Peptides, 28, 435-440.

Lockinger, A., Koberle, D., St. Konig, P., Saria, A., Herold, M., Cornelissen, G., Halzberg, F. (2004) Neuropeptide chronomics in clinically healthy adults:circacaotohoran and circadian patterns. Peptides, 25, 533-542. 
Loh, D.H., Abad, C., Colwell, C.S., Waschek, J.A. (2008) Vasoactive intestinal peptide is critical for circadian regulation of glucocorticoids. Neuroendocrinology, 88(4),246255.

Lovallo, W.R., Farag, N.H., Vincent, A.S., Thomas, T.L., Wilson, M.F. (2006)

Cortisol responses to mental stress, exercise, and meals following caffeine intake in men and women. Pharmacology, Biochemistry and Behavior, 83, 441-447,

Lovejoy, D.A. (2005) Neuroendocrinology: An integrated approach. John Wiley and Sons, Inc.

Lundberg, J.M., Anggard, A., Fahrenkrug, J., Hokfelt, T., Mutt, V. (1980)

Vasoactive intestinal polypeptide in cholinergic neurons of exocrine glands: Functional significance of coexisting transmitters for vasodilation and secretion. Proceedings of the National Academy of Sciences, 77(3), 1651-1685.

MacKenzie, T., Comi, R., Sluss, P., Keisari, R., Manwar, S., Kim, J., Larson, R., Baron, J.A. (2007) Metabolic and hormonal effects of caffeine: randomized, doubleblind, placebo-controlled crossover trial. Metabolism Clinical and Experimental, 56, 1694-1698.

Marini, M., Roda, G. (2000) Neuropeptide enzyme hydrolysis in human saliva. Archives of Oral Biology, 45, 775-786.

Marini, M., Roda, G. (2000) Enkephalin-degrading enzymes and their inhibitors in human saliva. Peptides, 21, 125-135.

McCorry, L.K. (2007) Physiology of the autonomic nervous system. American Journal of Pharmaceutical Education, 71(4), 1-11.

McDougall, S.J., Widdop, R. E., Lawrence, A. J. (2005) Central autonomic integration of psychological stressors: Focus on cardiovascular modulation. Autonomic Neuroscience: Basic and Clinical. 123, 1-11.

McEwen, B. (2002) The neurobiology and neuroendocrinology of stress: Implications for post-traumatic stress disorder from a basic science perspective. Psychiatric Clinics of North America, 25,469-494.

McEwen, B. (2009) The brain is the Central Organ of Stress and Adaptation.

Neuroimage. 47,911-913. 
Moldow, R.L., Bergen, M., Belin, K., Bululu, L., Couso, O., McLaughlin, J., Short, K.R., Servatius, R.J. (2006) Developing psychological profiles for monitoring stress. In Proceedings of SPIE, Enabling Technologies and Design of Nonlethal Weapons, Shwaery, G., Blitch, J., Land, C. 6219,6219K, SPIE, Orlando, Florida.

Morgan III, C.A., Wang, S., Mason, J., Southwick, S.M., Fox, P., Hazlett, G., Charney, D., Greenfield, G. (2000) Hormone profiles in humans experiencing military survival training. Biological Psychiatry, 47, 891-901.

Morgan, C.A., Wang, S., Southwick, S., Rasmusson, A., Hazlett, G., Hauger, R., Charney, D. (2000) Plasma Neuropeptide-Y concentrations in humans exposed to military survival training. Biological Psychiatry, 47, 902-909.

Morgan, C.A., Rasmusson, A, Wang, S., Hoyt, G., Hauger, R., Hazlett, G. (2002) Neuropeptide-Y, Cortisol, and subjective distress in humans exposed to acute stress: Replication and extension of previous report. Biological Psychiatry, 52, 136-142.

Morgan III, C.A., Southwick, S., Hazlett, G., Rasmusson, A., Hoyt, G., Zimolo, Z., Charney, D. (2004) Relationships among plasma dehydroepiandrosterone sulfate and cortisol levels, symptoms of dissociation and objective performance in humans exposed to acute stress. Archives of General Psychiatry, 61, 819-825.

Morgan, C.A, Rasmusson, A., Pietrzak, R.H., Coric, V., Southwick, S.M. (2009) Relationship among plasma dehydroepiandrosterone and dehydroepiandrosterone sulfate, cortisol, symptoms of dissociation, and objective performance in humans exposed to underwater navigation stress. Biological Psychiatry, 66, 334-340.

Naito, T., Itoh, H., Takeyama, M. (2003) Effects of Hange-koboku-to (Banxia-houpotang) on Neuropeptide levels in human plasma and saliva. Biological Pharmacology Bulletin, 26(11), 1609-1613.

Nakamura, H., Moroji, T., Nakamura, H., Nohara, S., Okada, A. (1992) Effects of whole-body vibration exposure on DOPAC and VIP-like immunoreactivity in the rat brain. Nippon Eiseigaku Zasshi, 46, 1095-1103.

Nakamura, H., Moroji, T., Nagase, H., Okazawa, T., Okada, H. (1994) Changes of cerebral vasoactive intestinal polypeptide and somatostatin-like immunoreactivity induced by noise and whole-body vibration in the rat. European Journal of Applied Physiology and Occupational Physiology, 68, 62-67.

Nater, U.M., Rohleder, N. Gaab, J., Berger, S., Jud, A., Kirschbaum, C., Ehlert, U. (2005) Human salivary alpha-amylase reactivity in a psychosocial stress paradigm. International Journal of Psychophysiology, 55, 333-342. 
Nater, U.M., LaMarca, R., Florin, L., Moses, A., Langhans, W., Koller, M., Ehlert, U. (2006) Stress-induced changes in human salivary alpha-amylase activity-associations with adrenergic activity. Psychoneuroendocrinology, 31, 49-58.

Nater, U.M., Rohleder, N., Schlotz, W., Ehlert, U., Kirschbaum, C. (2007)

Determinants of the diurnal course of salivary alpha-amylase.

Psychoneuroendocrinology, 32, 392-401.

Nater, U.M., Rohleder, N. (2009) Salivary alpha-amylase as a non-invasive biomarker for the sympathetic nervous system: Current state of research.

Psychoneuroendocrinology, 34, 486-496.

Naumova, EA., Sandulescu, T., Al Khatib, P., Thie, M., Lee, W.K., Zimmer, S. Arnold, W.H. (2012) Acute short-term mental stress does not influence salivary flow rate dynamics. PLos ONE, 7(12), e51323.

Netherton, C.T., Gooyer, I., Tamplin, A., Herbert, J. (2004) Salivary cortisol and dehydroepiandrosterone in relation to puberty and gender. Psychoneuroendocrinology, 29, $125-140$.

Nussdorfer, G.G., Mazzocchi, G. (1987) Vasoactive intestinal peptide (VIP) stimulates aldosterone secretion by rat adrenal glands invivo. Journal of Steroid Biochemistry, 26, 203-205.

Nussdorfer, G.G., Malendowicz, L.K. (1998) Role of VIP, PACAP, and Peptides in the regulation of the Hypothalamo-Pituitary Adrenal Axis. Peptides, 19(8), 1443-1467.

Obayashi, K. (2013) Salivary mental stress proteins. Clinica Chimica Acta, 425, 196201.

O'Dorisio, M.S., Hauger, M., O'Dorisio, T.M. (2002) Age-dependent levels of plasma neuropeptides in normal children. Regulatory Peptides, 109, 189-192.

Ogawa, Y., Kanai-Azuma, M., Akimoto, Y., Kawakami, H., Yanoshita, R. (2008)

Exosome-like vesicles with dipeptidyl peptidase IV in human saliva. Biological Pharmaceutical Bulletin. 31(6), 1059-1062.

Oktedalen, O., Opstad, P.K., Schaffalitzky de Muckadell, O.B. (1983-1) The plasma concentrations of secretin and vasoactive intestinal polypeptide (VIP) after long term, strenuous exercise. European Journal Physiology and Occupational Physiology, 52(1), 58. 
Oktedalen, O., Opstad, P.K., Fahrenkrug, J., Fonnum, F. (1983-2) Plasma concentration of vasoactive intestinal polypeptide during prolonged physical exercise, calorie supply deficiency, and sleep deprivation. Scandavian Journal of Gastroenterology, 18(8), 1057-1062.

Oliva, D., Nicosia, S., Spada, A., Giannatasio, G. (1982) VIP stimulates ACTH release and adenylate cyclase in human ACTH secreting pituitary adenomas. European. Journal of. Pharmacology, 83, 101-105.

Opstad, P.K. (1987) The plasma vasoactive intestinal peptide (VIP) reponse to exercise is increased after prolonged strain, sleep and energy deficiency and extinguished by glucose infusion. Peptides, 8(1), 175-178.

Papacosta, E., Nassis, G.P. (2011) Saliva as a tool for monitoring steroid, peptide and immune markers in sport and exercise science. Journal of Science and Medicine in Sport. 14, 424-434.

Petkov, V., Mosgoeller, W., Ziesche, R., Raderer, M., Stiebellehner, L., Vonbank, K., Funk, G.C., Hamilton, G., Novotny, C., Burian, B., Block, L.H. (2003) Vasoactive intestinal peptide as a new drug for treatment of primary pulmonary hypertension. The Journal of Clinical Investigation. 111(9), 1339-1346.

Piggins, H.D., Cutler, D.J. (2003) The roles of vasoactive intestinal polypeptide in mammalian circadian clock. Journal of Endocrinology. 177(1), 7-15.

Porges, S. (1995) Cardiac Vagal Tone: A Physiological Index of Stress. Neuroendocrine and Biobehavioral Reviews. 19(2), 225-233.

Porges, S.W. (2009) The Polyvagal theory: New Insights into Adaptive Reactions of the Autonomic Nervous System. Cleveland Clinic Journal of Medicine, 76(S2),S86-S90.

Preuss, D., Schoofs D., Schlotz, W., Wolf, O.T. (2010) The stressed student: influence of written examination and oral presentations on salivary cortisol concentrations in university students. Stress, 13(3), 221-229.

Proctor, G.B., Carpenter, G.H. (2007) Regulation of salivary gland function by autonomic nerves. Autonomic Neuroscience: Basic and Clinical, 133, 3-18.

Quinlan, P., Lane, J., Aspinall, L. (1997) Effects of hot tea, coffee and water ingestion on physiological response and mood: the role of caffeine, water and beverage type.

Psychopharmacology, 134, 164-173. 
Rabkin, J.G., McElhiney,M.C., Rabkin, R., McGrath, P.J., Ferrando, S.J. (2006) Placebo controlled trial of dehydroepiandrosterone (DHEA) for treatment of non-major depression in patients with HIV/AIDS. American Journal of Psychiatry, 163, 59-66.

Recordati, G. (2003) A thermodynamic model of the sympathetic and parasympathetic nervous systems. Autonomic Neuroscience: Basic and Clinical, 103, 1-12.

Rohleder, N., Nater, U.M., Wolf, J.M., Ehlert, U., Kirschbaum, C. (2004) Psychosocial stress-induced activation of salivary alpha-amylase. Annals of the New York Academy of Sciences, 1032, 258-263.

Rohleder, N., Kirschbaum, C. (2007) Effects of nutrition on neuro-endocrine stress responses. Current Opinion Clinical Nutrition Metabolism Care, 10, 504-510.

Rohleder, N., Nater, U.M. (2009) Determinants of salivary alpha-amylase in humans and methodological considerations. Psychoneuroendocrinology, 34, 469-485.

Rolandi. E., Franceschini, R., Cataldi, A., Barreca, T. (1990) Twenty-four-hour secretory pattern of vasoactive intestinal polypeptide in the elderly. Gerontology, 36, 356-360.

Sahar, T., Shalev, A.T., Porges, S.W. (2001) Vagal modulation of responses to mental challenges in posttraumatic stress disorder. Biological Psychiatry, 49, 637-643.

Said, S.I., Mutt, V. (1970) Polypeptide with broad biological activity: Isolation from small intestine. Science, 169(3951), 1217-1218.

Santavirta, N., Konttinen, Y., Tornwall, J., Segerberg, M., Santavirta, S., MatucciCerinic, M., Bjorvell, H. (1997) Neuropeptides of the autonomic nervous system in Sjogren's syndrome. Annals of the Rheumatic Diseases, 56(12), 737-740.

Schommer, N.C., Hellhammer, D.H., Kirschbaum, C. (2003) Dissociation between reactivity of the hypothalamus-piyuitary-adreno-medullary system to repeated psychosocial stress. Psychosomatic medicine, 65, 450-460.

Schoofs, D. Hartmann, R., Wolf, O.T. (2008) Neuroendocrine stress responses to an oral academic examination: No strong influence of sex, repeated participation and personality traits. Stress, 11(1), 52-61.

Schultheiss, O.C. (2013) Effect of sugarless chewing gum as a stimulant on progesterone, cortisol, and testosterone concentrations assessed in saliva. International Journal of Psychophysiology, 87, 111-114. 
Shepard, J.D., Mustafa, A., Whitsett, T.L., Passey, R.B., Lovallo, W. (2000) Additive pressor effects of caffeine and stress in male medical students at rick for hypertension. American Journal of Hypertension, 13, 475-481,

Shirotsuki, K., Izawa, S., Sugaya, N., Yamada, C.K., Ogawa, N., Ouchi, Y., Nagano, Y., Nomura, S. (2009) Salivary cortisol and DHEA reactivity to psychosocial stress in socially anxious males. International Journal of Psychophysiology, 72, 198-203.

Slivka, D., Hailes, W., Cuddy, J., Ruby, B. (2008) Caffeine and carbohydrate supplementation during exercise when in negative energy balance:effects on performance, metabolism, and salivary cortisol. Applied Physiology Nutrition Metabolism. 33, 1079-1085.

Spielberger, C.D. (1983) Manual for the State-Trait Anxiety Inventory, Consulting Psychologists.

Strahler, J., Berndt, C., Kirschbaum, C., Rohleder, N. (2010) Aging diurnal rhythms and chronic stress: Distinct alteration of diurnal rhythmicity of salivary alpha-amylase and cortisol. Biological Psychology, 84(2), 248-256.

Strand, F. (1999) Neuropeptides. The MIT Press. p.383.

Takai, N., Yamaguchi, M., Aragaki, T., Eto, K., Uchihashi, K., Nishikawa, Y. (2004) Effect of psychological stress on the salivary cortisol and amylase levels in healthy young adults. Archives of Oral Biology, 49, 963-968.

Tatemoto, K., Carlquist, M., Mutt, V. (1982) Neuropeptide Y: a novel brain peptide with structural similarity to peptide YY and pancreatic polypeptide. Nature, 296, 659660.

Thayer, R.E. (1978) Factor analytic and reliability studies on the activation-deactivation adjective checklist. Psychological Reports, 42, 747-756.

Thomadaki, K., Helmerhorst, E.J., Tian, N., Sun, X., Siqueira, W.L., Walt, D.R., Oppenheim, F.G. (2011) Whole-saliva proteolysis and its impact on salivary diagnostics. Journal of Dental Research, 90(11), 1325-1330.

Tsigos, C., Chrousos, G. (2002) Hypothalamic-pituitary-adrenal axis, neuroendocrine factors and stress. Journal of Psychosomatic Research, 53, 865-871.

van Stegeren, A., Rohleder, N., Everaerd, W., Wolf, O.T. (2006) Salivary alpha amylase as marker for adrenergic activity during stress: Effect of betablockade. Psychoneuroendocrinology, 31, 137-141. 
Ventre, G., Colonna, C.,Smith, J., Alfano, D., Moldow, R. (2013) Salivary VIP concentrations are elevated in humans after acute stress. Peptides, 49, 27-31.

Vosko, A.M., Schroeder, A., Loh, D.H., Colwell, C.S. (2007) Vasoactive intestinal peptide and the mammalian circadian system. General and Comparative Endocrinology, 152, $165-175$.

Wolkowitz, O.M., Reus, V.I., Keebler, A., Nelson, N., Friedland, M., Brizendine, L., Roberts, E. (1999) Double-blind treatment of major depression with dehydroepiandrosterone. American Journal of Psychiatry, 156, 646-649.

Yehuda, R., Brand, S., Yank, R.K. (2006) Plasma Neuropeptide Y concentrations in combat exposed veterans: Relationship to trauma exposure, recovery from PTSD, and coping. Biological Psychiatry, 59, 660-663.

Zukowska-Grojec, Z., Karwatoska-Prokopczuk, E., Rose, W., Rone, J., Movaforgher, S., Ji, H. (1998) Neuropeptide Y: a novel angiogenic factor from the sympathetic nerves and endothelium. Circulation Research. 83, 187-195. 